\title{
TOWARD THE STANDARD POPULATION SYNTHESIS MODEL OF THE X-RAY BACKGROUND: EVOLUTION OF X-RAY LUMINOSITY AND ABSORPTION FUNCTIONS OF ACTIVE GALACTIC NUCLEI INCLUDING COMPTON-THICK POPULATIONS
}

\author{
Yoshihiro Ueda ${ }^{1}$, Masayuki Akiyama $^{2}$, Günther Hasinger ${ }^{3}$, Takamitsu Miyaji ${ }^{4,5,7}$, and Michael G. Watson ${ }^{6}$ \\ ${ }^{1}$ Department of Astronomy, Kyoto University, Kitashirakawa-Oiwake-cho, Sakyo-ku, Kyoto 606-8502, Japan \\ ${ }^{2}$ Astronomical Institute, Tohoku University, 6-3 Aramaki, Aoba-ku, Sendai 980-8578, Japan \\ ${ }^{3}$ Institute for Astronomy, 2680 Woodlawn Drive Honolulu, HI 96822-1839, USA \\ ${ }^{4}$ Instituto de Astronomía, Universidad Nacional Autónoma de México, Ensenada, Baja California, Mexico \\ ${ }^{5}$ University of California, San Diego, Center for Astrophysics and Space Sciences, 9500 Gilman Drive, La Jolla, CA 92093-0424, USA \\ ${ }^{6}$ Department of Physics and Astronomy, University of Leicester, University Road, Leicester LE1 7RH, UK \\ Received 2013 September 15; accepted 2014 March 18; published 2014 April 22
}

\begin{abstract}
We present the most up to date X-ray luminosity function (XLF) and absorption function of active galactic nuclei (AGNs) over the redshift range from 0 to 5 , utilizing the largest, highly complete sample ever available obtained from surveys performed with Swift/BAT, MAXI, ASCA, XMM-Newton, Chandra, and ROSAT. The combined sample, including that of the Subaru/XMM-Newton Deep Survey, consists of 4039 detections in the soft $(0.5-2 \mathrm{keV})$ and/or hard $(>2 \mathrm{keV}$ ) band. We utilize a maximum likelihood method to reproduce the count rate versus redshift distribution for each survey, by taking into account the evolution of the absorbed fraction, the contribution from Compton-thick (CTK) AGNs, and broadband spectra of AGNs, including reflection components from tori based on the luminosity- and redshift-dependent unified scheme. We find that the shape of the XLF at $z \sim 1-3$ is significantly different from that in the local universe, for which the luminosity-dependent density evolution model gives much better description than the luminosity and density evolution model. These results establish the standard population synthesis model of the X-ray background (XRB), which well reproduces the source counts, the observed fractions of CTK AGNs, and the spectrum of the hard XRB. The number ratio of CTK AGNs to the absorbed Compton-thin (CTN) AGNs is constrained to be $\approx 0.5-1.6$ to produce the $20-50 \mathrm{keV}$ XRB intensity within present uncertainties, by assuming that they follow the same evolution as CTN AGNs. The growth history of supermassive black holes is discussed based on the new AGN bolometric luminosity function.
\end{abstract}

Key words: diffuse radiation - galaxies: active - quasars: general - surveys - X-rays: diffuse background

Online-only material: color figures

\section{INTRODUCTION}

Understanding the cosmological evolution of supermassive black holes (SMBHs) is a key issue in modern astrophysics. The good correlation of the mass of an SMBH in a galactic center with that of the bulge in the present-day universe (e.g., Magorrian et al. 1998; Ferrarese \& Merritt 2000; Gebhardt et al. 2000; Marconi \& Hunt 2003; Häring \& Rix 2004; Hopkins et al. 2007; Kormendy \& Bender 2009; Gültekin et al. 2009) indicates that SMBHs and galaxies co-evolved in the past. This idea is also supported by the similarity of the global history between star formation and SMBH growth (Marconi et al. 2004). The so-called downsizing or anti-hierarchical evolution, the trend that more massive systems formed in earlier cosmic time, has been revealed for both SMBHs (Ueda et al. 2003, hereafter U03; Hasinger et al. 2005, hereafter H05) and galaxies (e.g., Cowie et al. 1996; Kodama et al. 2004; Fontanot et al. 2009).

Active galactic nuclei (AGNs) are the phenomena where SMBHs gain their masses from accreting gas by converting a part of their gravitational energies into radiation. It is known that the majority of AGNs are obscured by gas and dust surrounding the SMBHs, being classified as "type-2" AGNs. To elucidate the growth history of SMBHs, a complete survey of AGNs, including heavily obscured populations throughout the history of the universe, is necessary. X-ray observations, in particular, those at high energies above a few $\mathrm{keV}$, provide

\footnotetext{
7 Instituto de Astronomía, UNAM, P.O. Box 439027, San Ysidro, CA 92143-9027, USA.
}

one of the most powerful approaches for AGN detection, thanks to the strong penetrating power against absorption and little contamination from star lights in the host galaxies. Furthermore, the deepest X-ray surveys currently available achieve the highest sensitivity even for unobscured ("type-1") AGNs among those at any wavelengths (see Brandt \& Hasinger 2005 and references therein). The surface number density of the faintest X-ray AGNs reaches $\sim 10^{4} \mathrm{deg}^{-2}$ (Xue et al. 2011).

The integration of emission from all accreting SMBHs in the universe is observed as the X-ray background (XRB). To quantitatively solve the XRB origin is equivalent to revealing the cosmological evolution of AGNs that constitute the XRB. The XRB spans over a wide energy range, from $\sim 0.1 \mathrm{keV}$ to $\sim 100 \mathrm{keV}$ and then is smoothly connected to the gamma-ray background at higher energies. Its spectrum has a peak energy density around $\sim 20 \mathrm{keV}$. At energies below $\sim 8 \mathrm{keV}$, now almost all of the XRB is resolved into discrete sources, mainly AGNs. Enormous efforts have been made to identify AGNs detected in X-ray surveys on the basis of multiwavelength observations, and the redshifts (and hence luminosities) of a large fraction of these sources are now estimated. These results make it possible to determine the spatial number density of AGNs that constitute the XRB below $\sim 8 \mathrm{keV}$ and its evolution. By contrast, in the hard $\mathrm{X}$-ray band above $\sim 10 \mathrm{keV}$, a significant fraction of the XRB is still left unresolved. Therefore, at present, the whole origin of the XRB over the wide range cannot be directly revealed by resolving individual sources.

It is very important to construct a "population synthesis model" of the XRB,where the evolutions of all X-ray emitting 
AGNs with various types are formulated (for previous works, see, e.g., Comastri et al. 1995; Gilli et al. 1999, 2007; Ueda et al. 2003; Ballantyne et al. 2006; Treister et al. 2009). The model, in principle, must explain all of the observational constraints, including source number counts, redshift and luminosity distribution, and the shape of the XRB. Once established, it gives the basis to understand the accretion history of the universe traced by X-rays, which is subject to least biases.

Two major elements in the population synthesis models are the X-ray luminosity function (XLF) and absorption function (or $N_{\mathrm{H}}$ function, Ueda et al. 2003) of AGNs. The XLF represents the number density of AGNs per comoving space as a function of luminosity and redshift, one of the most important statistical quantities that can be determined from unbiased surveys. Previously, many studies have been made on the cosmological evolutions of the XLF of AGNs in the soft band below $2 \mathrm{keV}$ (e.g., Maccacaro et al. 1991; Boyle et al. 1993; Jones et al. 1997; Page et al. 1997; Miyaji et al. 2000; Hasinger et al. 2005) and the hard band above $2 \mathrm{keV}$ (e.g., Ueda et al. 2003; La Franca et al. 2005; Barger et al. 2005; Silverman et al. 2008; Ebrero et al. 2009; Yencho et al. 2009; Aird et al. 2010). By using hard X-ray selected samples that contain both type-1 (unabsorbed) and type-2 (absorbed) AGNs, the absorption functions have also been investigated (Ueda et al. 2003; La Franca et al. 2005; Ballantyne et al. 2006; Treister \& Urry 2006; Hasinger 2008). Besides the well-established anti-correlation of absorption fraction with luminosity (e.g., Ueda et al. 2003; Steffen et al. 2003; Simpson 2005), several works have reported that the fraction of absorbed AGNs increased toward higher redshift from $z=0$ to $z>1$ (La Franca et al. 2005; Ballantyne et al. 2006; Treister \& Urry 2006; Hasinger 2008). More recent studies of high-redshift AGNs at $z>2$ in deep fields (Hiroi et al. 2012; Iwasawa et al. 2012) reveal larger absorption fractions of high-luminosity AGNs compared with the local universe. This redshift evolution is not included in the population synthesis model by Gilli et al. (2007), one of the most widely referred models available at present.

There still remain uncertainties on the evolution of AGNs, however. The first issue is the shape of the XLF and its cosmological evolution. On the basis of hard X-ray surveys, Ueda et al. (2003), Silverman et al. (2008), Ebrero et al. (2009), and Yencho et al. (2009) find that the XLF of AGNs is best described with the luminosity-dependent density evolution (LDDE) model. Later, Aird et al. (2010) propose that the luminosity and density evolution (LADE) where the shape of the XLF is constant over the whole redshift range, unlike the case of LDDE, also gives a similarly good fit to their data. While the downsizing behavior is commonly seen in both models, quite a different number of AGNs are predicted, particularly at high redshifts of $z \geqslant 3$. The second one is the number density (or fraction) of heavily obscured AGNs with $N_{\mathrm{H}}>10^{24} \mathrm{~cm}^{-2}$, so-called Compton-thick AGNs (CTK AGNs). Even hard X-ray surveys above $10 \mathrm{keV}$ are subject to bias against detecting heavily CTK AGNs because the transmitted emission is significantly suppressed due to repeated Compton scattering (see, e.g., Wilman \& Fabian 1999; Ikeda et al. 2009). It is not easy to identify individual CTK AGNs with limited photon statistics in deep survey data and to estimate their intrinsic number density by correcting for such biases.

In this work, we present our latest results of AGN XLF over the redshift range from 0 to 5 , by utilizing one of the largest combined samples ever available, obtained from surveys of various depths, widths, and energy bands performed with Swift/BAT, the Monitor of All-sky X-ray Image (MAXI; Matsuoka et al. 2009), ASCA, XMM-Newton, Chandra, and ROSAT. The sample consists of 4039 detections in the soft $(0.5-2 \mathrm{keV})$ and/or hard ( $>2 \mathrm{keV})$ bands. We utilize a maximum likelihood (ML) method to reproduce the count rate versus redshift distribution for each survey by taking into account the selection biases. In the analysis, the contribution of CTK AGNs is considered, which is found to be more important in harder bands and at fainter fluxes. This enables us to determine the intrinsic XLF and the absorption function of type-1 plus type- 2 AGNs with an unprecedented accuracy, thus establishing a standard population synthesis model of the XRB that is consistent with most observational constraints currently available.

The organization of the paper is as follows. Section 2 explains the sample used in our analysis. In Section 3, the absorption properties of AGNs are discussed and the absorption function is formulated. Section 4 introduces the "template model" of broadband X-ray spectra of AGNs adopted in this work. The distribution of photon index is examined by using the Swift/BAT sample in Section 5. Section 6 describes the details of main analysis using the whole sample. The best-fit results of the XLF are presented there. The predictions from the population synthesis model are given in Section 7. We also discuss the constraints on the CTK AGN fraction and degeneracy with other parameters. Section 8 represents a determination of the bolometric luminosity function (BLF) of AGNs based on our new XLF and the growth history of SMBHs. The conclusions of our work are summarized in Section 9. The cosmological parameters of $\left(H_{0}, \Omega_{\mathrm{m}}, \Omega_{\lambda}\right)=\left(70 h_{70} \mathrm{~km} \mathrm{~s}^{-1} \mathrm{Mpc}^{-1}, 0.3,0.7\right)$ are adopted throughout the paper. The "log" symbol represents the base-10 logarithm, while "In" the natural logarithm.

\section{SAMPLE}

In order to investigate the XLF and absorption function of AGNs covering a wide range of luminosity and redshift, it is vital to construct a sample combined from various surveys with different flux limits and area. Also, high degrees of identification completeness in terms of spectroscopic and/or photometric redshift determination are required to minimize systematic uncertainties caused by sample incompleteness. Basically, $\mathrm{X}$-ray surveys at higher energies are more suitable to detect obscured AGNs with less biases. Nevertheless, those in the soft band $(\approx 0.5-2 \mathrm{keV})$ are also quite useful, as long as such biases are properly corrected. Generally, fainter flux limits are achieved in softer energy bands, thanks to the larger collecting area of X-ray telescopes. In particular, for high-redshift AGNs, the reduction of observed fluxes due to photo-electric absorptions becomes less important, thanks to the $K$-correction effect. Indeed, the soft X-ray surveys are often utilized to search for high-redshift AGNs, including type-2 objects (e.g., Miyaji et al. 2000; Silverman et al. 2008).

In our study, we collect the results from surveys performed with Swift/BAT, MAXI, ASCA, XMM-Newton, Chandra, and $R O S A T$, by utilizing the heritage of X-ray astronomy accumulated up to the present. Only those with high identification completeness $(\gtrsim 90 \%)$ are included. Our sample is composed of those from the Swift/BAT 9 month survey (BAT9; Tueller et al. 2008), MAXI 7 month survey (MAXI7; Hiroi et al. 2011; Ueda et al. 2011), ASCA Medium Sensitivity Survey (AMSS; Ueda et al. 2001; Akiyama et al. 2003) and Large Sky Survey (ALSS; 
Ueda et al. 1999; Akiyama et al. 2000), Subaru/XMM-Newton Deep Survey (SXDS; Ueda et al. 2008; M. Akiyama et al. 2014, in preparation), XMM-Newton survey of the Lockman Hole (LH/XMM; Hasinger et al. 2001; Brunner et al. 2008), HELLAS2XMM survey (H2X; Fiore et al. 2003), Hard Bright Serendipitous Sample (HBSS) in the XMM-Newton Bright Survey (XBS; Della Ceca et al. 2004, 2008), Chandra Large Area Synoptic X-ray Survey (CLASXS; Yang et al. 2004; Steffen et al. 2004; Trouille et al. 2008), Chandra Lockman Area North Survey (CLANS; Trouille et al. 2008, 2009), Chandra Deep Survey North (CDFN; Alexander et al. 2003; Barger et al. 2003; Trouille et al. 2008) and South (CDFS; Xue et al. 2011), and various ROSAT surveys (see Miyaji et al. 2000 and H05 and references therein).

This paper presents the first work that makes use of the large X-ray sample in the SXDS (Furusawa et al. 2008; Ueda et al. 2008), one of the wide and deep multiwavelength survey projects with a comparable survey area and depth to the Cosmic Evolution Survey (COSMOS; Scoville et al. 2007), in order to constrain the XLF of AGNs with the best statistical accuracy. Also, new hard X-ray all-sky surveys with Swift/BAT and MAXI are utilized instead of HEAOI AGN samples that were usually employed in previous studies. The other soft-band and hard-band samples adopted here are also analyzed in $\mathrm{H} 05$ and Hasinger (2008), respectively, although in some cases, different selection criteria are applied in our analysis to increase the completeness (see below). The major difference in the soft-band sample from that used by $\mathrm{H} 05$ is that we include both type- 1 and type-2 AGNs because we aim to investigate the evolution of the whole AGN population. We do not use the sample from the Serendipitous Extragalactic X-ray Source Identification program (Eckart et al. 2006) adopted by Hasinger (2008), whose redshift identification completeness is slightly worse $(\sim 84 \%)$ than our threshold. The sample of the XMM-Newton Mediumsensitivity Survey (Barcons et al. 2007) used in Hasinger (2008) is discarded because it partially overlaps with the SXDS sample. A detailed description of each survey is given below.

\subsection{Swift/BAT}

We utilize the Swift/BAT 9 month catalog (Tueller et al. 2008), which originally contains 137 non-blazar AGNs with a flux limit of $2 \times 10^{-11} \mathrm{erg} \mathrm{cm}^{-2} \mathrm{~s}^{-1}$ in the $14-195 \mathrm{keV}$ band with a signal-to-noise ratio $(\mathrm{S} / \mathrm{N})>4.0$. The Swift/BAT survey, performed at energies above $10 \mathrm{keV}$, provides us with the least biased AGN sample in the local universe against obscuration, except for heavily CTK sources absorbed with $N_{\mathrm{H}} \gg 10^{24} \mathrm{~cm}^{-2}$ along with those of INTEGRAL (e.g., Beckmann et al. 2009). X-ray spectra below $10 \mathrm{keV}$ of all the AGNs in the sample have been obtained from extensive follow up observations by other missions such as Swift/XRT, XMM-Newton, and Suzaku (e.g., Winter et al. 2009a, 2009b; Eguchi et al. 2009). Thus, we can discuss their statistical properties without incompleteness problems. Here, we refer to the absorption column densities summarized in Table 1 of Ichikawa et al. (2012), where new results obtained from broadband X-ray spectra of Suzaku are included. To define a statistical sample that is consistent with the survey area curve given in Figure 1 of Tueller et al. (2008), we further impose criteria where (1) the Galactic latitude is larger than $15 \operatorname{deg}\left(|b|>15^{\circ}\right)$ and (2) $\mathrm{S} / \mathrm{N}>4.8$. We regard the pair of the interacting galaxies NGC 6921 and MCG +04-48-002 unresolved with Swift/BAT as one source, adopting the spectral information below $10 \mathrm{keV}$ of the latter. The sample consists of 87 non-blazar AGNs with an identification completeness of $100 \%$.

\section{2. $M A X I$}

We include the local AGN sample from the MAXI extragalactic survey performed in the $4-10 \mathrm{keV}$ band (Ueda et al. 2011) in our analysis. While the sample significantly overlaps with the Swift/BAT 9 month sample, it is useful to directly constrain the XLF in the $2-10 \mathrm{keV}$ band. The sample is collected from the first MAXI/GSC catalog at high Galactic latitudes $\left(|b|>10^{\circ}\right)$ with a flux limit of $1.5 \times 10^{-11} \mathrm{erg} \mathrm{cm}^{-2} \mathrm{~s}^{-1}$ (4-10 keV) based on the first 7 months of data (Hiroi et al. 2011). It consists of 37 non-blazar AGNs, by excluding Cen A and ESO 509-066, and is used by Ueda et al. (2011) to calculate the XLF and absorption function of AGNs in the local universe. A merit is its high completeness of identification (99.3\%), and similar to the Swift/BAT 9 month catalog, the information of the $\mathrm{X}$-ray spectra below $10 \mathrm{keV}$ is available for all of the objects, as summarized in Table 1 of Ueda et al. (2011). Unlike in U03, we do not use the local AGN samples by the HEAOl mission (Piccinotti et al. 1982), considering the lower completeness than in the MAXI sample and systematic uncertainties of the measured fluxes close to the sensitivity limit that must be corrected for (see Shinozaki et al. 2006). Note that only 17 objects out of the total MAXI/GSC AGNs are listed in the Piccinotti et al. (1982) sample, suggesting significant long-term variabilities of nearby AGNs over $30 \mathrm{yr}$.

\subsection{ASCA}

$A S C A$, the fourth Japanese X-ray astronomical satellite, performed first imaging surveys in the energy band above $2 \mathrm{keV}$ and provided statistical X-ray samples at $>100$ times deeper levels than that of the HEAO1 A2 survey. These are still very useful to bridge the flux range between all-sky surveys and deep surveys with Chandra and XMM-Newton. In our paper, we utilize the two major samples, the ALSS and AMSS; both are first utilized by U03 to calculate the hard XLF. The ALSS covers a continuous area of $5.5 \mathrm{deg}^{2}$ near the North Galactic Pole, and a flux limit of $\approx 1 \times 10^{-13} \mathrm{erg} \mathrm{cm}^{-2} \mathrm{~s}^{-1}(2-10 \mathrm{keV})$ is achieved (Ueda et al. 1998, 1999). Thirty AGNs detected with the SIS instrument are optically identified by Akiyama et al. (2000) with $100 \%$ completeness by ignoring the one unidentified source in Akiyama et al. (2000) as a fake detection (see Section 2.2.2 of U03). The AMSS is based on a serendipitous X-ray survey with the GIS instrument, whose catalogs are published in Ueda et al. (2001) and Ueda et al. (2005). We use the "AMSSn" (Akiyama et al. 2003) and "AMSSs" samples, for which systematic identification programs were carried out in the northern $\left(\right.$ DEC $\left.>20^{\circ}\right)$ and southern sky $\left(\right.$ DEC $\left.<-20^{\circ}\right)$, respectively. The AMSSn and AMSSs samples contain 74 and 20 optically identified non-blazar AGNs, respectively, with a detection significance larger than $5.5 \sigma$ at the flux range between $5 \times 10^{-12} \mathrm{erg} \mathrm{cm}^{-2} \mathrm{~s}^{-1}$ and $3 \times 10^{-13}$ in the $2-10 \mathrm{keV}$ band. Three $\mathrm{X}$-ray sources are left unidentified, and thus the completeness is $97 \%$. The total survey area covered by the AMSSn and AMSSs is $90.8 \mathrm{deg}^{2}$. We utilize the hardness ratio between the 2-10 (or 2-7) $\mathrm{keV}$ and $0.7-2 \mathrm{keV}$ bands to estimate the absorption or photon index of each source in the ALSS and AMSS, while results from follow up observations with ASCA or XMM-Newton are available for six and two sources in the ALSS and AMSS, respectively (see Section 2.2 of U03). 


\subsection{XMM-Newton}

\subsubsection{SXDS}

The SXDS is one of the largest multiwavelength surveys, covering from radio to $\mathrm{X}$-rays, with an unprecedented combination of depth and sky area. The SXDS field is a contiguous region of $>1 \mathrm{deg}^{2}$ centered at R.A. $=02^{\mathrm{h}} 18^{\mathrm{m}}$ and decl. $=$ $-05^{\mathrm{d}} 00^{\mathrm{m}}(\mathrm{J} 2000)$. The large survey area makes it possible to establish the statistical properties of extragalactic populations without being affected by cosmic variance and is also very useful to construct a sample of sources with small surface densities, like high-luminosity AGNs (i.e., QSOs) at high redshifts. The optical photometric catalogs in the $B, V, R, i^{\prime}$, and $z^{\prime}$ bands obtained with Subaru/Suprime-Cam are presented in Furusawa et al. (2008), achieving the $3 \sigma$ sensitivities of $B=28.4, V=$ 27.8, $R_{c}=27.7, i^{\prime}=27.7$, and $z^{\prime}=26.6$ (for the 2 arcsec aperture in the $\mathrm{AB}$ magnitudes), respectively. The deep imaging data are particularly important to reliably identify high-redshift AGNs by photometric redshifts.

The X-ray catalog of the SXDS (Ueda et al. 2008) is based on the EPIC data in the $0.3-10 \mathrm{keV}$ band obtained from seven pointings performed with XMM-Newton, which covers a total of $1.14 \mathrm{deg}^{2}$. It contains $866,1114,645$, and 136 sources with sensitivity limits of $6 \times 10^{-16}, 8 \times 10^{-16}, 3 \times 10^{-15}$, and $5 \times 10^{-15} \mathrm{erg} \mathrm{cm}^{-2} \mathrm{~s}^{-1}$ in the $0.5-2,0.5-4.5,2-10$, and 4.5-10 keV bands, respectively, with a detection likelihood $\geqslant 7$. The deep optical images were taken by five pointing with Suprime-Cam with a slightly different shape from that of the combined XMM-Newton image. By limiting to the area of $1.02 \mathrm{deg}^{2}$ where these optical imaging data are available, we define two independent parent samples consisting of 584 and 781 sources detected in the $2-10 \mathrm{keV}$ and $0.5-2 \mathrm{keV}$ bands, respectively.

The results from multiwavelength identification of the X-ray sources in the SXDS is presented in M. Akiyama et al. (2014, in preparation). Out of the $584(2-10 \mathrm{keV})$ and $781(0.5-2 \mathrm{keV})$ $\mathrm{X}$-ray source samples, 576 and 733 objects are left as AGN candidates, respectively, after excluding Galactic stars, stellar objects close to bright galaxies, and clusters of galaxies. Among them, 397 and 514 targets have spectroscopic redshift based on near-infrared and/optical data. For the rest, M. Akiyama et al. (2014, in preparation) determine the photometric redshifts, except for seven (2-10 keV) and eight (0.5-2 keV) objects for which the fluxes in less than four bands are available. The redshifts are estimated on the basis of HyperZ photometric redshift code with the spectral energy distribution (SED) templates of galaxies and QSOs. In addition, constraints from the morphology and absolute magnitude limits for the galaxy and QSO templates are applied (see Hiroi et al. 2012 and M. Akiyama et al. 2014, in preparation, for details). The accuracy of the photometric redshifts is found to be $\Delta z /\left(1+z_{\text {spec }}\right)=0.06$ as a median value. Finally, 569 and 725 AGNs detected in the 2-10 keV and $0.5-2 \mathrm{keV}$ bands have either spectroscopic or photometric redshifts, among which 412 are common sources. The identification completeness is rather high, $99 \%$ both for the $2-10 \mathrm{keV}$ and $0.5-2 \mathrm{keV}$ samples. The hardness ratio between the $2-10 \mathrm{keV}$ and $0.5-2 \mathrm{keV}$ is used to estimate the absorption or photon index of each source.

\subsubsection{Lockman Hole}

We use both the hard-band-(2-4.5 keV) and soft-band(0.5-2 keV) selected samples from the XMM-Newton deep survey of the Lockman Hole (Hasinger et al. 2001; Brunner et al. 2008). They are essentially the same as those used in the analysis by Hasinger (2008) and H05, respectively, except for small differences: (1) type-2 AGNs detected in the soft band are also included in our analysis, unlike the case of H05 who only used type-1 AGNs, and (2) some unpublished photometric redshifts for a few hard-band-selected sources mentioned in Section 2.8 of Hasinger (2008) are not utilized and are instead treated as unidentified objects in our analysis. The effects by the latter difference are negligible. Before the AO-2 phase, 17 observations of this field were performed with XMM-Newton. The X-ray source catalog is provided by Brunner et al. (2008) from $637 \mathrm{ks}$ (hard band) and the $770 \mathrm{ks}$ (soft band) data sets. To maximize the completeness of redshift identification, subsamples are defined from two offaxis intervals with different flux limits, as detailed in Hasinger (2008) and H05. The survey area after this selection becomes $0.183 \mathrm{deg}^{2}$ and $0.126 \mathrm{deg}^{2}$ with fainter flux limits of $6.1 \times$ $10^{-15} \mathrm{erg} \mathrm{cm}^{-2} \mathrm{~s}^{-1}(2-10 \mathrm{keV})$ and $1.3 \times 10^{-15} \mathrm{erg} \mathrm{cm}^{-2} \mathrm{~s}^{-1}$ $(0.5-2 \mathrm{keV})$ for the hard- and soft-band samples, respectively. The soft- (hard-) band sample consists of 57 (58) AGNs having either spectroscopic or photometric redshifts (Fotopoulou et al. 2012) with identification completeness of $91 \%$ (88\%). We refer to Mateos et al. (2005) for the results of X-ray spectral analysis, with some updates for sources whose redshifts were revised after the publication of Mateos et al. (2005) (A. Streblyanska 2006, private communication).

\subsubsection{HELLAS2XMM}

The HELLAS2XMM (Baldi et al. 2002) is a serendipitous survey performed with XMM-Newton in the $2-10 \mathrm{keV}$ band. We basically refer to the original sample complied by Fiore et al. (2003) selected from five XMM-Newton fields, covering a total area of $0.9 \mathrm{deg}^{2}$. Among the 122 hard X-ray selected sources, Fiore et al. (2003) present the spectroscopic redshifts for 97 AGNs. Additional information on the redshifts of the unidentified sources in the original catalog of Fiore et al. (2003) is reported in Mignoli et al. (2004) and Maiolino et al. (2006). To ensure a high completeness, we apply a flux limit of $1.5 \times 10^{-14} \mathrm{erg} \mathrm{cm}^{-2} \mathrm{~s}^{-1}(2-10 \mathrm{keV})$ band, which leaves 95 sources after excluding one Galactic star and one extended object. Among them, 87 AGNs have redshifts and 8 objects are left unidentified. The completeness is thus $92 \%$. The results of X-ray spectral analysis are presented in Perola et al. (2004). Unlike in Hasinger (2008), we do not include the new catalog of HELLAS2XMM by Cocchia et al. (2007) in our analysis, considering the lower rate of redshift identification (59 out of 110 sources).

\subsubsection{Hard Bright Survey}

The HBSS (Della Ceca et al. 2004) is a subsample detected in the 4.5-7.5 keV band from those of the XBS, aiming at relatively bright X-ray sources. From an area of $25 \mathrm{deg}^{2}$ at $|b|>20^{\circ}$, Della Ceca et al. (2008) define a complete fluxlimited sample consisting of 67 sources with MOS2 count rates larger than 0.002 count $\mathrm{s}^{-1}(4.5-7.5 \mathrm{keV})$, corresponding to $2.2 \times 10^{-13} \mathrm{erg} \mathrm{cm}^{-2} \mathrm{~s}^{-1}$ in the $2-10 \mathrm{keV}$ band for a photon index of 1.6. The sample is optically identified except for 2 sources, and in total, 62 AGNs are cataloged in Della Ceca et al. (2008). The completeness of redshift identification is $97 \%$. The results from the spectral analysis of the XMM-Newton data are also summarized in Della Ceca et al. (2008). 


\subsection{Chandra}

\subsubsection{CLASXS}

The CLASXS is an intermediate-depth survey covering a continuous area of $\approx 0.4 \mathrm{deg}^{2}$ by 9 pointings in the Lockman Hole-Northwest field. Yang et al. (2004) present the X-ray source catalog containing 525 sources. Following the work by Steffen et al. (2004), Trouille et al. (2008) report spectroscopic redshifts for 260 sources that are not stars and photometric redshifts for 134 sources out of the 245 spectroscopically unidentified objects. We first select sources detected in the 2-8 keV band at small off-set angles, which are used to calculate the $\log N-\log S$ relation in their work (see their Figure 8). The survey area is thus reduced to be $0.28 \mathrm{deg}^{2}$. Then, to maximize the completeness, we further impose a threshold to the hard-band count rate above $6.6 \times 10^{-4}$ counts $\mathrm{s}^{-1}$, corresponding to $1.9 \times 10^{-14} \mathrm{erg} \mathrm{cm}^{-2} \mathrm{~s}^{-1}$ in the $2-10 \mathrm{keV}$ band for a photon index of 1.2. This leaves a total of 50 identified AGNs and 2 unidentified sources after excluding 1 Galactic star. The redshift completeness is $96.2 \%$. We use the hardness ratio between the $2-8 \mathrm{keV}$ and $0.4-2 \mathrm{keV}$ bands to estimate the absorption or photon index of each source in Section 2.8 .

\subsubsection{CLANS}

The CLANS is another intermediate-depth survey in the Lockman Hole region, consisting of nine separated Chandra pointings with an exposure of $\approx 70 \mathrm{ks}$ for each. The field is a part of the of the Spitzer Wide-Area Infrared Extragalactic Survey and covers a total area of $0.6 \mathrm{deg}^{2}$. A total of $761 \mathrm{X}$-ray sources are cataloged in Trouille et al. (2008), with flux limits of $3.5 \times 10^{-15} \mathrm{erg} \mathrm{cm}^{-2} \mathrm{~s}^{-1}$ and $7 \times 10^{-16} \mathrm{erg} \mathrm{cm}^{-2} \mathrm{~s}^{-1}$ in the $2-8 \mathrm{keV}$ and $0.5-2 \mathrm{keV}$ bands, respectively. Trouille et al. (2008) also provide 327 spectroscopic redshifts (except for stars) and 234 photometric redshifts out of the 425 spectroscopically unidentified objects. Some of the redshifts are updated in Trouille et al. (2009). For our analysis, we only select sources that are detected at offset angles from the pointing center smaller than $8^{\prime}$ and have $\mathrm{S} / \mathrm{Ns}$ larger than 3 in the hard $(2-8 \mathrm{keV})$ or soft $(0.5-2 \mathrm{keV})$ band. Further, to achieve high completeness of redshift identification (95\%), we impose flux limits of $1.0 \times$ $10^{-14} \mathrm{erg} \mathrm{cm}^{-2} \mathrm{~s}^{-1}(2-10 \mathrm{keV})$ and $2.3 \times 10^{-15} \mathrm{erg} \mathrm{cm}^{-2} \mathrm{~s}^{-1}$ $(0.5-2 \mathrm{keV})$ by assuming a photon index of 1.2 and 1.4 for the hard-band and soft-band samples, which leaves 159 and 191 identified AGNs with 8 and 10 unidentified objects other than 0 and 3 Galactic stars, respectively. Above these flux limits, the survey area can be regarded as constant at $0.490 \mathrm{deg}^{2}$ (see their Figure 5). The number of common AGNs in the two samples are 122.

\subsection{3. $C D F N$}

The CDFN is currently the second deepest survey ever performed in X-rays after the CDFS. The 2 Ms catalog containing 503 sources from a survey area of $0.124 \mathrm{deg}^{2}$ is presented by Alexander et al. (2003). To define the hard-band-(2-8 keV) and soft-band-(0.5-2 keV) selected samples presented in Alexander et al. (2003), we apply a threshold of $\geqslant 3$ to the $S / N$, defined as the count rate divided by its negative error in each band. The sensitivities are $2.8 \times 10^{-17} \mathrm{erg}^{-2} \mathrm{sm}^{-1}$ and $2.1 \times 10^{-16} \mathrm{erg} \mathrm{cm}^{-2} \mathrm{~s}^{-1}$ in the $0.5-2 \mathrm{keV}$ and $2-10 \mathrm{keV}$ bands, converted from the count rate limits by assuming a photon index of 1.4 and 1.0, respectively. We basically refer to the redshift catalog provided by Trouille et al. (2008), which contains the results from previous works, including Barger et al.
(2003), Swinbank et al. (2004), Chapman et al. (2005), and Reddy et al. (2006). In total, Trouille et al. (2008) report 307 spectroscopic redshifts (except for stars) and 107 photometric redshifts out of the 182 spectroscopically unidentified objects. To further increase the completeness of redshift identification, we also utilize the compilation of both spectroscopic and photometric redshifts by G. Hasinger (2008, private communication) for the remaining unidentified objects, which is used in Hasinger (2008). The hard- and soft-band samples consist of 286 and 375 AGN candidates (including galaxies) with redshift identification (with 179 and 252 spectroscopic redshifts), respectively, leaving no unidentified objects. The number of common sources in the two samples is 195 . The completeness is thus $100 \%$ for both samples. The hardness ratio between the $2-8 \mathrm{keV}$ and $0.5-2 \mathrm{keV}$ count rates is used to estimate the absorption or photon index of each source in Section 2.8. The hard-band sample is the same as used by Hasinger (2008), except that we do not apply the off-axis cut in the sample selection to increase the sample size. Unlike in $\mathrm{H} 05$, we do not limit the soft-band sample to only type-1 AGNs.

\subsection{4. $C D F S$}

The CDFS provides the deepest X-ray survey data set ever performed to date obtained from a total of $4 \mathrm{Ms}$ exposures of Chandra. We use the source catalog complied by Xue et al. (2011). It lists $740 \mathrm{X}$-ray sources from an area of $464.5 \mathrm{arcmin}^{2}$. We define the hard-band-(2-8 keV) and soft-band-(0.5-2 keV) selected samples that satisfy the binomial probability source selection criterion of $P<0.004$ in each survey band. They consist of 375 and 626 sources with flux limits of $1.1 \times$ $10^{-16} \mathrm{erg} \mathrm{cm}^{-2} \mathrm{~s}^{-1}(2-10 \mathrm{keV})$ and $1.1 \times 10^{-17} \mathrm{erg} \mathrm{cm}^{-2} \mathrm{~s}^{-1}$ $(0.5-2 \mathrm{keV})$, converted from the count rate limits by assuming a photon index of 1.0 and 1.4 , respectively. We basically refer to the redshift identification presented in Xue et al. (2011) but adopt the new result reported by Vito et al. (2013) for five $z>3$ AGNs (four spectroscopic and one photometric redshifts). Extended sources and those identified as "Star" are excluded. We keep "AGN" and "Galaxy" types in both hard and soft samples before filtering them by the count rate and redshift according to the procedure described in Section 2.7 because the latter may well be actual AGNs in some cases (see Xue et al. 2011). The hardand soft-band samples consist of 358 and 583 redshift-identified (228 and 378 spectroscopically identified) AGN candidates with 17 and 43 objects without redshift information, thus achieving the completeness of $96 \%$ and $93 \%$, respectively. The number of common sources in both samples is 313 . The hardness ratio between the two bands is utilized to estimate the absorption or photon index in Section 2.8.

\subsection{ROSAT}

We utilize a large, soft X-ray selected AGN sample obtained from various ROSAT surveys with different depth and area to cover a brighter flux range than those of Chandra and $X M M-N e w t o n$. Essentially, the same sample was utilized by Miyaji et al. (2000) and H05 to construct the soft XLF. It is selected from the ROSAT Bright Survey (RBS; Schwope et al. 2000), the RASS Selected-Area Survey North (SA-N; Appenzeller et al. 1998), the ROSAT North Ecliptic Pole Survey (NEPS; Gioia et al. 2003; Mullis et al. 2004), the ROSAT International X-ray/Optical Survey (RIXOS; Mason et al. 2000), and the ROSAT Medium Survey (RMS). We refer the reader to Miyaji et al. (2000) and H05 for a detailed description of each survey. For our analysis, we impose a conservative flux cut of $S \geqslant 3.5 \times 10^{-14} \mathrm{erg} \mathrm{cm}^{-2} \mathrm{~s}^{-1}$, below which a sufficiently 
Table 1

Surveys Used in the Analysis

\begin{tabular}{|c|c|c|c|c|c|c|}
\hline Survey & $\begin{array}{c}\text { Energy Band } \\
(\mathrm{keV})\end{array}$ & 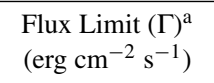 & $\begin{array}{c}\text { Area } \\
\left(\operatorname{deg}^{2}\right)\end{array}$ & No. of $\mathrm{AGNs}^{\mathrm{b}}$ & $\begin{array}{c}\text { Completeness } \\
(\%)\end{array}$ & Reference \\
\hline BAT9 & $14-195$ & $8.4 \times 10^{-12}(1.6)$ & 30500 & $87(85)$ & 100 & (1) \\
\hline MAXI7 & $4-10$ & $2.4 \times 10^{-11}(1.6)$ & 34000 & $38(37)$ & 99 & (2) \\
\hline AMSS & $2-10$ & $3.1 \times 10^{-13}(1.6)$ & 90.8 & $95(95)$ & 97 & (3) \\
\hline ALSS & $2-7$ & $1.2 \times 10^{-13}(1.6)$ & 5.56 & $30(30)$ & 100 & (4) \\
\hline SXDS & $2-10$ & $2.7 \times 10^{-15}(1.2)$ & 1.02 & $569(567)$ & 99 & (5) \\
\hline SXDS & $0.5-2$ & $6.8 \times 10^{-16}(1.4)$ & 1.02 & $725(703)$ & 99 & (5) \\
\hline LH/XMM & $2-4.5$ & $6.1 \times 10^{-15}(1.2)$ & 0.183 & $57(57)$ & 91 & (6) \\
\hline LH/XMM & $0.5-2$ & $1.3 \times 10^{-15}(1.4)$ & 0.126 & $58(57)$ & 88 & (7) \\
\hline $\mathrm{H} 2 \mathrm{X}$ & $2-10$ & $1.7 \times 10^{-14}(1.2)$ & 0.90 & $87(87)$ & 92 & (8) \\
\hline HBSS & $4.5-7.5$ & $2.2 \times 10^{-13}(1.6)$ & 25.17 & $62(62)$ & 97 & (9) \\
\hline CLASXS & 2-8 & $1.9 \times 10^{-14}(1.2)$ & 0.280 & $50(50)$ & 96 & (10) \\
\hline CLANS & $2-8$ & $1.0 \times 10^{-14}(1.2)$ & 0.490 & $159(158)$ & 95 & $(10,11)$ \\
\hline CLANS & $0.5-2$ & $2.3 \times 10^{-15}(1.4)$ & 0.490 & $191(183)$ & 95 & $(10,11)$ \\
\hline CDFN & $2-8$ & $2.1 \times 10^{-16}(1.0)$ & 0.124 & 307 (298) & 100 & $(6,10)$ \\
\hline CDFN & $0.5-2$ & $2.8 \times 10^{-17}(1.4)$ & 0.124 & $402(234)$ & 100 & $(7,10)$ \\
\hline CDFS & $2-8$ & $1.1 \times 10^{-16}(1.0)$ & 0.129 & $358(347)$ & 95 & (12) \\
\hline CDFS & $0.5-2$ & $1.1 \times 10^{-17}(1.4)$ & 0.129 & $583(299)$ & 93 & (12) \\
\hline$R O S A T^{\mathrm{c}}$ & $0.5-2$ & $3.5 \times 10^{-14}(1.8)$ & $0.3-21400$ & $722(705)$ & 99 & (7) \\
\hline
\end{tabular}

Notes.

a The smallest source flux in each sample, given in the $2-10 \mathrm{keV}$ band for the hard-band surveys above $2 \mathrm{keV}$ (including BAT9) and in the $0.5-2 \mathrm{keV}$ band for the soft-band surveys. The photon index assumed to convert the count rate into the flux is shown in the parentheses.

b The number of AGNs at $z=0.002-5.0$ after excluding sources with count rates smaller than $c_{\text {lim }}(z)$ (see Section 2.8).

c The completeness is calculated from the RBS, SA-N, NEPS, and RIXOS samples.

References. (1) Tueller et al. 2008; (2) Ueda et al. 2011; (3) Akiyama et al. 2003; (4) Akiyama et al. 2000; (5) M. Akiyama et al. 2014, in preparation; (6) Hasinger 2008 and references therein; (7) Hasinger et al. 2005 and references therein;

(9) Della Ceca et al. 2008; (10) Trouille et al. 2008; (11) Trouille et al. 2009; (12) Xue et al. 2011.

large number of sources are available from the Chandra and $X M M-N e w t o n$ surveys. Unlike in H05, we include both type1 and type- 2 AGNs in our analysis, as done in Miyaji et al. (2000). In total, 722 AGNs are sampled. Information of the $\mathrm{X}$-ray spectra covering the $2-10 \mathrm{keV}$ band is unavailable for most of the sources. In our main analysis (Section 6), we find that the source counts of the ROSAT AGNs are better reproduced than by any models when we adopt slightly $(\approx 10 \%-20 \%)$ higher fluxes than the original ones. We consider that this is probably due to the cross-calibration error in the absolute flux between different missions (see, e.g., Ueda et al. 1999). To deal with this issue, we adopt $15 \%$ higher fluxes than those reported in the original tables for all ROSAT sources. The uncertainty does not affect the determination of the parameters and the XLF and absorption function within the errors, however.

\subsection{Sample Summary}

Table 1 summarizes the energy band, sensitivity limits, survey area, number of sources with measured redshifts, and identification completeness for each survey. Here, the completeness is defined as the fraction of all sources with redshift identification in the total sample (including non-AGNs) selected with the same detection criteria. ${ }^{8}$ In total, we have 87 detections in the very hard band (14-195 keV), 1791 in the hard band (within $2-10 \mathrm{keV})$, and 2654 in the soft band (0.5-2 keV). Although common sources are contained in multiple samples obtained in different energy bands of the same field, we basically treat them as independent detections in our main analysis (Section 6).

\footnotetext{
8 In the case of SXDS, CDFN, and CDFS, it refers to the fraction of AGNs and galaxies with spectroscopic or photometric redshifts in the total AGN candidates.
}

The flux limits in units of erg $\mathrm{cm}^{-2} \mathrm{~s}^{-1}$ are converted from the vignetting corrected count rate limit in each survey band by assuming a power-law photon index, given in the parentheses of the third column. In the following analysis, we correct for the incompleteness of each survey by multiplying the area by the completeness fraction, independently of flux. This procedure implicitly assumes that unidentified sources follow the same redshift and luminosity distribution as identified sources. Although it is a simplified assumption, possible uncertainties in the correction little affect the overall determination of the XLF, thanks to the high completeness of our sample.

Figure 1 plots the survey area against flux in the $2-10 \mathrm{keV}$ and $0.5-2 \mathrm{keV}$ bands. That of the Swift/BAT survey is overlaid in Figure 1 (left) by the red curve. The $\log N-\log S$ relations in the integral form are shown in Figure 2 (left) for the hard band and Figure 2 (right) for the soft band. In the former, the Swift/BAT sample is not included. The data at $S=2.4 \times 10^{-11}-2.4 \times 10^{-12} \mathrm{erg} \mathrm{cm}^{-2} \mathrm{~s}^{-1}$ are not shown because of the survey flux gap in the hard band. The photon indices listed in Table 1 for each survey are assumed for the count rate to flux conversion in Figures 1 and 2.

For the analysis of XLF presented in Section 6, we limit the redshift range to $z=0.002-5.0$. Although this lower limit is smaller than $z=0.015$ adopted by previous works (e.g., Miyaji et al. 2000, U03, H05), we confirm that excluding nearby AGNs at $z<0.015$ from the analysis does not change our results over the statistical errors, and hence possible effects of the local over-density can be ignored. In very deep surveys like CDFN and CDFS, we find non-negligible contamination from starburst galaxies in our sample at the lowest luminosity range, which would affect our analysis of the XLF. To exclude such sources by not relying on their type identifications in the catalog 

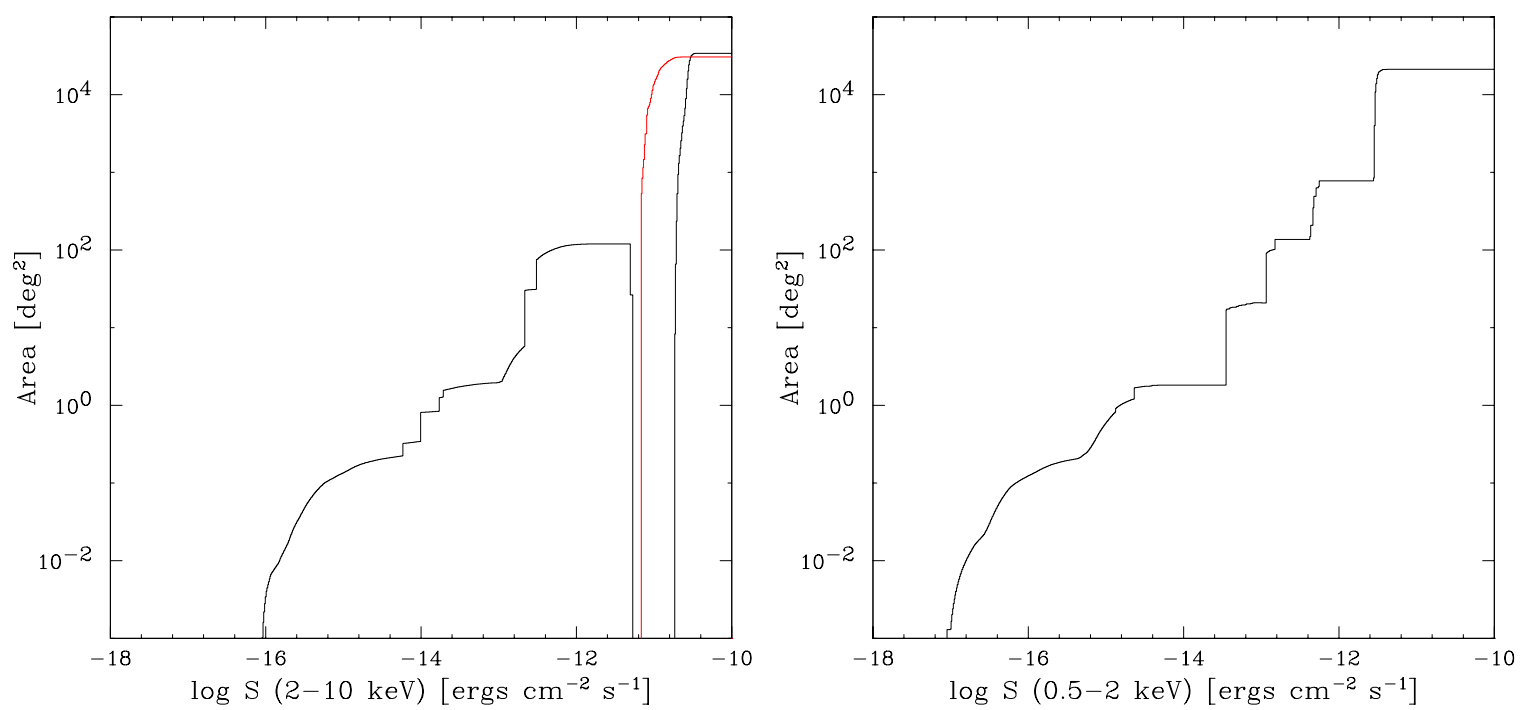

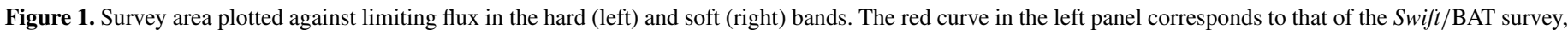
assuming $\gamma=1.6$.

(A color version of this figure is available in the online journal.)
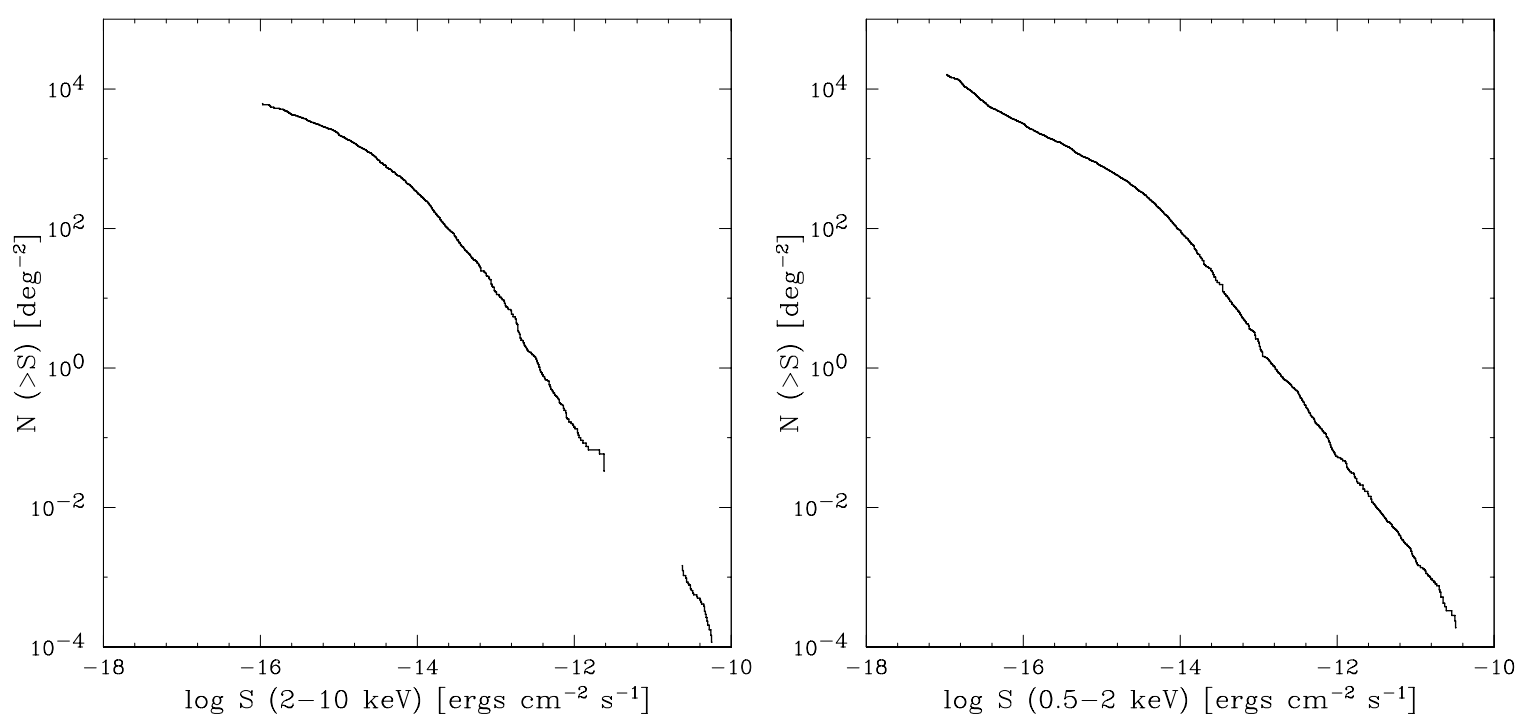

Figure 2. Observed source counts in the hard (left) and soft (right) bands.

that could be quite uncertain (Xue et al. 2011), we impose a lower limit of the count rate as a function of redshift in each survey, $c_{\text {lim }}(z)$, that corresponds to $L_{X}=10^{41} \mathrm{erg} \mathrm{s}^{-1}$ or $L_{X}=$ $10^{42} \mathrm{erg} \mathrm{s}^{-1}$ for the hard-band or soft-band sample, respectively. We adopt a lower value for the $L_{\mathrm{X}}$ threshold for the hard-band sample because emission from starburst galaxies is generally softer than that from AGNs, although X-ray binaries could significantly contribute to the hard X-ray luminosity in galaxies with very high star formation rates and/or stellar masses (e.g., Persic \& Raphaeli 2002; Lehmer et al. 2010). We confirm that increasing the lower limit to $L_{X}=10^{41.5} \mathrm{erg} \mathrm{s}^{-1}$ in the hard band does not change the XLF parameters over the errors. To be conservative, $c_{\text {lim }}(z)$ is calculated by assuming $\Gamma=1.9$ and no absorption. Applying these cuts, in addition to the redshift limit $(z=0.002-5.0)$, slightly reduces the number of sources in each sample, which are listed in the fifth column of Table 1. The numbers of detections used in our main analysis (Section 6) thus become 85 in the very hard band (above $10 \mathrm{keV}$ ), 1770 in the hard band (within 2-10 keV), 2184 in the soft band (below $2 \mathrm{keV}$ ), and hence 4039 in total.

\subsection{Estimate of Luminosity}

For convenience, here we calculate an intrinsic (de-absorbed) luminosity in the rest-frame $2-10 \mathrm{keV}$ band, $L_{\mathrm{X}}$, for each object, following the same procedure as described in Section 3.2 of U03. It can be calculated as

$$
L_{\mathrm{X}}=4 \pi d_{\mathrm{L}}(z)^{2} F_{\mathrm{X}}
$$

where $d_{\mathrm{L}}(z)$ is the luminosity distance at the source redshift $z$, and $F_{\mathrm{X}}$ is the de-absorbed flux in the observer's frame $2 /(1+z)-10 /(1+z) \mathrm{keV}$ band. To convert the count rate into $F_{X}$, we need to consider the spectrum of each source by taking into account the energy response of the instrument used in the survey. We refer to the results of individual spectral analysis in terms of absorption and photon index whenever such information is available. As for the Swift/BAT sample, which contains identified CTK AGNs, we assume the "template spectra" described in Section 4 with the $N_{\mathrm{H}}$ values available in Table 1 of Ichikawa et al. (2012) and a photon index of 1.94 for X-ray type-1 AGNs (with $\log N_{\mathrm{H}}<22$ ) or 1.84 for 

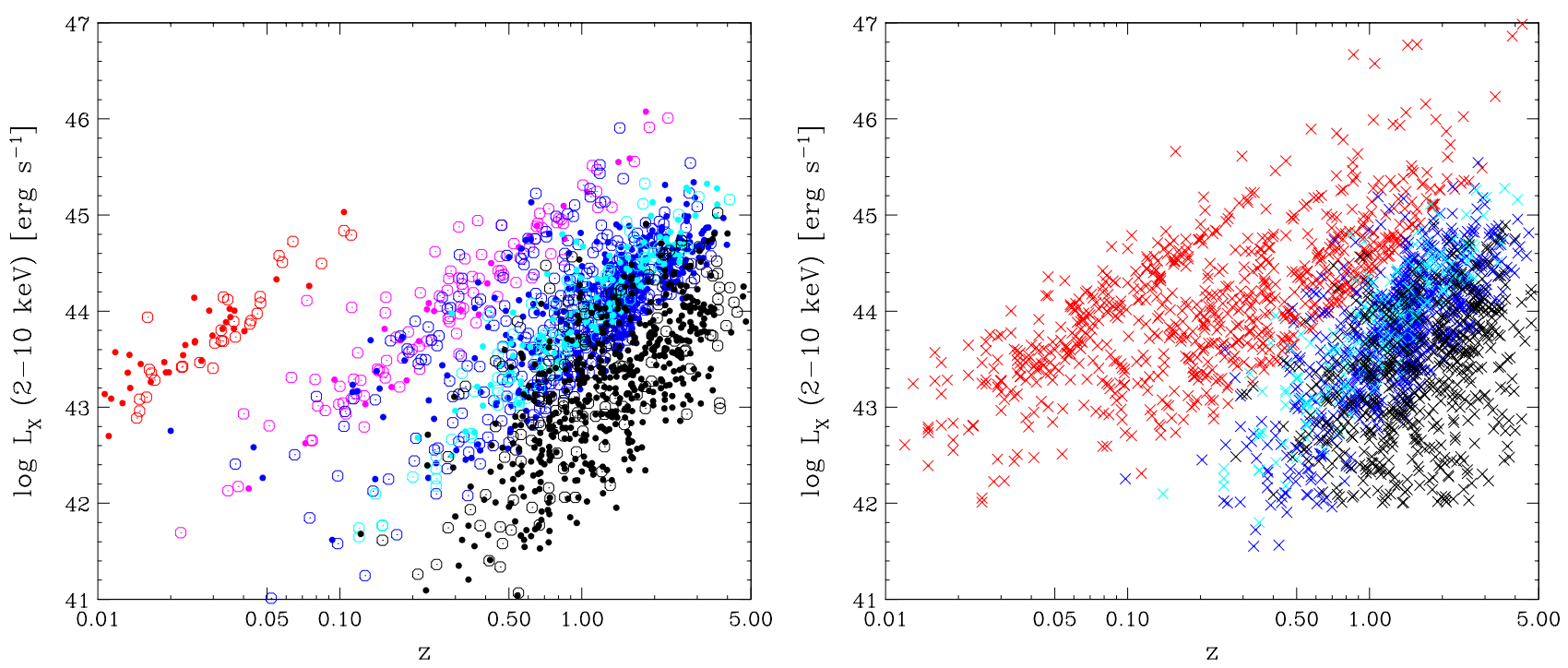

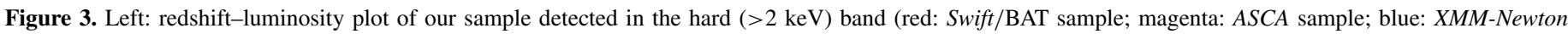

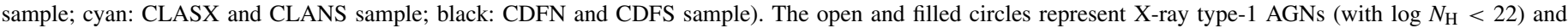

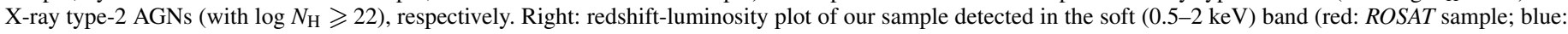
$X M M-N e w t o n$ sample; cyan: CLANS sample; black: CDFN and CDFS sample).

(A color version of this figure is available in the online journal.)

$\mathrm{X}$-ray type-2 AGNs (with $\log N_{\mathrm{H}} \geqslant 22$ ). For the rest, we utilize the hardness ratio between the hard- and soft-band count rates to estimate the absorption or photon index. In this case, we assume a cut-off power-law model in the form of $E^{-\Gamma} \exp \left(E / E_{\mathrm{c}}\right)$, where $E_{\mathrm{c}}=300 \mathrm{keV}$ plus its reflection component from cold matter calculated with the pexrav code (Magdziarz \& Zdziarski 1995) for the intrinsic spectrum. In the pexrav model, the solar abundances, an inclination angle of $\theta_{\text {inc }}=60^{\circ}$, and a reflection strength of $R_{\mathrm{tot}} \equiv \Omega / 2 \pi=1.0$ are adopted. If the hardness ratio is found to be larger than that expected from $\Gamma=1.9$, then we determine the intrinsic absorption $N_{\mathrm{H}}$ by assuming $\Gamma=1.9$. Otherwise, we calculate a corresponding photon index by assuming no absorption. The base spectrum with $\Gamma=1.9$, $\theta_{\text {inc }}=60^{\circ}$, and $R_{\text {tot }}=1.0$ is the same as adopted in U03, which roughly corresponds to the averaged one of type- 1 and type- 2 AGNs in the template spectra (Section 4). Note that, with this procedure, we can only obtain absorptions of $\log N_{\mathrm{H}} \leqslant 24$. This means we ignore the possibility that an object is a CTK AGN, whose spectrum is quite complex and is not necessarily harder than Compton-thin (CTN) AGNs in the energy band below $10 \mathrm{keV}$, due to the relatively large contribution from other softer components than the transmitted one (see Section 4). Finally, for the case of the ROSAT surveys where hardness ratio information is not available, we simply assume $\Gamma=1.9$ and no absorption in the above continuum.

Figure 3 displays the $L_{X}$ versus redshift distribution for the hard-band (left) and soft-band (right) samples after the above count-rate selection is applied. The different colors correspond to different surveys. Here, the MAXI sample is not included in order to avoid the overlap with the Swift/BAT sample. In Figure 3 (left), AGNs that are found to be absorbed with $N_{\mathrm{H}}>$ $10^{22} \mathrm{~cm}^{-2}$ (X-ray type-2 AGNs) are marked by filled circles, while less absorbed ones (X-ray type-1 AGNs) are by open circles. We do not distinguish the two classes for the softband sample in Figure 3 (right), however, which includes many ROSAT sources. Note that in our main analysis in Section 6, we perform ML fitting directly to the list of the count rate (not luminosity) and redshift by considering a luminosity-dependent reflection component and different photon index for type- 1 and type-2 AGNs. The contribution of CTK AGNs is also taken into account there. Thus, the values of $L_{X}$ calculated here should be regarded as tentative, and they will be mainly used for plotting purposes.

\section{ABSORPTION DISTRIBUTION}

The absorption properties of AGNs are important to understand the circumnuclear environments, such as dusty "tori" surrounding the SMBH. In this section, we quantitatively formulate the absorption function (or $N_{\mathrm{H}}$ function) and its evolution, which must be taken into account in the analysis of the XLF presented in the next sections. The result is finally included in the population synthesis model of the XRB. We first determine the absorption function in the local universe by an analysis of the $N_{\mathrm{H}}$ distribution of the Swift/BAT 9 month sample. Then, its redshift evolution is examined by using the Swift/BAT, AMSS, and SXDS hard-band samples.

\subsection{Formulation}

Following U03 and later works, we introduce the absorption function or $N_{\mathrm{H}}$ function, $f\left(L_{\mathrm{x}}, z ; N_{\mathrm{H}}\right)$, the probability distribution function of absorption column density in the X-ray spectrum of an AGN at a given luminosity and a redshift, in units of $\left(\log N_{\mathrm{H}}\right)^{-1}$. We assume that it has no dependence on the photon index. By adopting the same definition of U03, the absorption function is normalized to unity in the "CTN" region of $\log N_{\mathrm{H}} \leqslant$ 24 so that

$$
\int_{20}^{24} f\left(L_{\mathrm{X}}, z ; N_{\mathrm{H}}\right) d \log N_{\mathrm{H}}=1 .
$$

The lower limit of $\log N_{\mathrm{H}}=20$ is a dummy value introduced for convenience, and we assign $\log N_{\mathrm{H}}=20$ for unabsorbed AGNs with $\log N_{\mathrm{H}}<20$. Note that $f\left(L_{\mathrm{x}}, z ; N_{\mathrm{H}}\right)$ is also defined for CTK AGNs with $\log N_{\mathrm{H}}>24$. The reason why we normalize the absorption function within $20 \leqslant \log N_{\mathrm{H}} \leqslant 24$ is that the XLF can be most accurately determined for CTN AGNs from the survey data. As done in the previous population synthesis models, we represent the number density of CTK AGNs in terms of the ratio to that of CTN AGNs at the same $L_{X}$ and $z$. 
The same formulation as presented in Ueda et al. (2011) is adopted for the shape of the absorption function. First, we introduce the $\psi\left(L_{\mathrm{X}}, z\right)$ parameter, which represents the fraction of absorbed CTN AGNs (i.e., $\log N_{\mathrm{H}}=22-24$ ) in total CTN AGNs (i.e., $\log N_{\mathrm{H}}=20-24$ ) as a function of $L_{\mathrm{X}}$ and $z$. It is expressed by a linear function of $\log L_{X}$ within a range between $\psi_{\min }$ and $\psi_{\max }$ :

$$
\begin{aligned}
\psi\left(L_{\mathrm{X}}, z\right)= & \min \left[\psi_{\max }, \max \left[\psi_{43.75}(z)\right.\right. \\
& \left.\left.-\beta\left(\log L_{\mathrm{X}}-43.75\right), \psi_{\min }\right]\right],
\end{aligned}
$$

where $\psi_{43.75}(z)$ gives the absorption fraction of CTN AGNs with $\log L_{\mathrm{X}}=43.75^{9}$ located at $z$. In this work, we adopt $\psi_{\min }=0.2$, the fraction of absorbed AGNs at highest luminosity range found in the Swift/BAT survey (Burlon et al. 2011), and $\psi_{\max }=0.84$, the upper limit from the assumption on the form of the absorption function explained below. On the basis of the results by Hasinger (2008), we take into account the redshift dependence as

$$
\psi_{43.75}(z)= \begin{cases}\psi_{43.75}^{0}(1+z)^{a 1} & {[z<2.0]} \\ \psi_{43.75}^{0}(1+2)^{a 1} & {[z \geqslant 2.0]}\end{cases}
$$

Here, $\psi_{43.75}^{0} \equiv \psi_{43.75}(z=0)$ is the local value, a free parameter to be determined from the analysis in the next subsection. In our paper, we adopt $\beta=0.24$, the best-fit value obtained by Ueda et al. (2011), which also agrees with those in various redshift ranges obtained by Hasinger (2008).

We define the absorption function separately for two ranges of the $\psi\left(L_{\mathrm{X}}, z\right)$ value:

$$
\begin{gathered}
\left(\text { for } \psi\left(L_{\mathrm{X}}, z\right)<\frac{1+\epsilon}{3+\epsilon}\right) \\
f\left(L_{\mathrm{X}}, z ; N_{\mathrm{H}}\right)= \begin{cases}1-\frac{2+\epsilon}{1+\epsilon} \psi\left(L_{\mathrm{X}}\right) & {\left[20 \leqslant \log N_{\mathrm{H}}<21\right]} \\
\frac{1}{1+\epsilon} \psi\left(L_{\mathrm{X}}\right) & {\left[21 \leqslant \log N_{\mathrm{H}}<22\right]} \\
\frac{1}{1+\epsilon} \psi\left(L_{\mathrm{X}}\right) & {\left[22 \leqslant \log N_{\mathrm{H}}<23\right]} \\
\frac{\epsilon}{1+\epsilon} \psi\left(L_{\mathrm{X}}\right) & {\left[23 \leqslant \log N_{\mathrm{H}}<24\right]} \\
\frac{f_{\mathrm{CTK}}}{2} \psi\left(L_{\mathrm{X}}\right) & {\left[24 \leqslant \log N_{\mathrm{H}}<26\right]}\end{cases}
\end{gathered}
$$

and

$$
\begin{gathered}
\left(\text { for } \psi\left(L_{\mathrm{X}}, z\right) \geqslant \frac{1+\epsilon}{3+\epsilon}\right) \\
f\left(L_{\mathrm{X}}, z ; N_{\mathrm{H}}\right)= \begin{cases}\frac{2}{3}-\frac{3+2 \epsilon}{3+3 \epsilon} \psi\left(L_{\mathrm{X}}\right) & {\left[20 \leqslant \log N_{\mathrm{H}}<21\right]} \\
\frac{1}{3}-\frac{\epsilon}{3+3 \epsilon} \psi\left(L_{\mathrm{X}}\right) & {\left[21 \leqslant \log N_{\mathrm{H}}<22\right]} \\
\frac{1}{1+\epsilon} \psi\left(L_{\mathrm{X}}\right) & {\left[22 \leqslant \log N_{\mathrm{H}}<23\right]} \\
\frac{\epsilon}{1+\epsilon} \psi\left(L_{\mathrm{X}}\right) & {\left[23 \leqslant \log N_{\mathrm{H}}<24\right]} \\
\frac{f_{\text {CTK }}^{2} \psi\left(L_{\mathrm{X}}\right)}{2} & {\left[24 \leqslant \log N_{\mathrm{H}}<26\right] .}\end{cases}
\end{gathered}
$$

The absorption function is flat above $\log N_{\mathrm{H}}=21$ in the former case (Equation (5)), while the value in $\log N_{\mathrm{H}}=21-22$ is set as the mean of those at $\log N_{\mathrm{H}}=20-21$ and $\log N_{\mathrm{H}}=22-23$ in the latter (Equation (6)). The fraction of CTK AGNs to the absorbed CTN AGNs (with $\log N_{\mathrm{H}}=22-24$ ) is given by the $f_{\text {Стк }}$ parameter, and its absorption function is assumed to be flat over the range of $\log N_{\mathrm{H}}=24-26$. The maximum absorption

\footnotetext{
9 Here, we change the reference luminosity from $\log L_{X}=44$ adopted in U03 and Ueda et al. (2011) to $\log L_{X}=43.75$ to match with the formulation by Hasinger (2008).
}

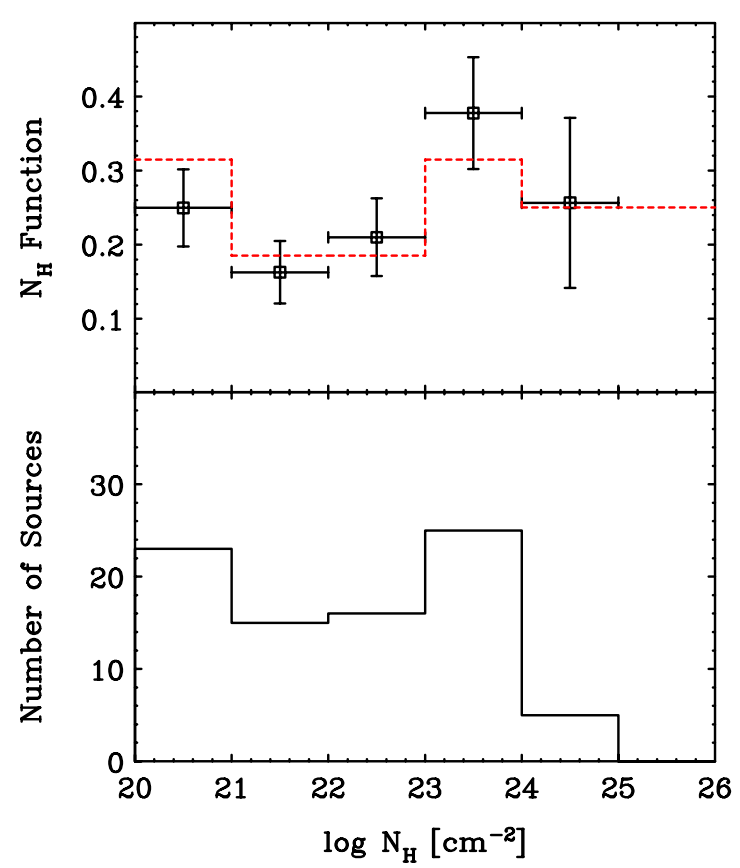

Figure 4. Observed histogram of $N_{\mathrm{H}}$ in the Swift/BAT sample (lower) and estimated $N_{\mathrm{H}}$ function corrected for detection biases (upper). The red lines represent an example of the $N_{\mathrm{H}}$ function we adopt.

(A color version of this figure is available in the online journal.)

fraction corresponds to the case of $f\left(N_{\mathrm{H}}\right)=0$ at $\log N_{\mathrm{H}}=20-21$ and thus $\psi_{\max }=(1+\epsilon) /(3+\epsilon)$. The $\epsilon$ parameter represents the ratio of the absorption function in $\log N_{\mathrm{H}}=23-24$ to that in $\log N_{\mathrm{H}}=22-23$, which is fixed at $\epsilon=1.7$ in our paper. This is the same value adopted by U03 and Gilli et al. (2007), based on the $N_{\mathrm{H}}$ distribution of [O-III] selected AGNs in the local universe (Risaliti et al. 1999). Although Ueda et al. (2011) adopt $\epsilon=1.3$ on the basis of the original $N_{\mathrm{H}}$ distribution of Swift/BAT 9 month sample reported in Tueller et al. (2008), we find that $\epsilon=1.7$ better fit the revised $N_{\mathrm{H}}$ distribution that utilizes new $N_{\mathrm{H}}$ measurements, as reported in Ichikawa et al. (2012). Recent work by Vasudevan et al. (2013), based on a deeper survey of Swift/BAT, also favors a larger value than that of Tueller et al. (2008).

\subsection{Absorption Function in the Local Universe}

To determine the absorption function in the local universe, we use the revised $N_{\mathrm{H}}$ distribution of the Swift/BAT 9 month sample based on Table 1 of Ichikawa et al. (2012). In this analysis, we limit the luminosity range to $\log L_{X}=42-46$, which leaves 84 AGNs. ${ }^{10}$ The lower panel of Figure 4 displays the observed histogram of the $N_{\mathrm{H}}$ distribution. Even if this sample is selected by hard X-rays above $10 \mathrm{keV}$ having strong penetrating power, there still remains detection biases against obscuration at large column densities due to the suppression of the hard X-ray flux (see Section 4), which becomes particularly significant in CTK AGNs. The presence of this bias in hard X-ray surveys is discussed by, e.g., Malizia et al. (2009) and Burlon et al. (2011). Importantly, the small observed fraction of CTK AGNs $(5 / 84 \approx 6 \%$ in our sample) does not mean that they are a minor population.

To derive the absorption function by correcting for these effects, we perform an ML fit of the absorption function with the same approach as described in Section 4.1 of U03. In this

\footnotetext{
${ }_{10}$ NGC 4395, NGC 4051, and NGC 4102 are excluded.
} 
Table 2

Parameters of Absorption Function

\begin{tabular}{lccccc}
\hline \hline$\psi_{43.75}^{0}$ & $\beta$ & $\psi_{\min }$ & $\epsilon$ & $a 1$ & $f_{\text {CTK }}$ \\
\hline $0.43 \pm 0.03^{\mathrm{a}}$ & 0.24 (fixed) & 0.2 (fixed) & 1.7 (fixed) & $0.48 \pm 0.05$ & 1.0 (fixed)
\end{tabular}

Notes. Errors are $1 \sigma$ for a single parameter.

${ }^{a}$ Errors are based on the Swift/BAT 9 month sample. It is fixed at the best-fit value when performing ML fit to the whole sample (Section 6).

analysis, the likelihood estimator, $L^{\prime}$, to be minimized through fitting is defined as

$$
L^{\prime}=-2 \sum_{i} \ln \frac{f\left(L_{\mathrm{X} i}, z_{i} ; N_{\mathrm{H} i}\right) \sum_{j} A_{\mathrm{j}}\left(N_{\mathrm{H} i}, \Gamma_{i}, L_{\mathrm{X} i}, z_{i}\right)}{\int f\left(L_{\mathrm{X} i}, z_{i} ; N_{\mathrm{H}}\right) \sum_{j} A_{\mathrm{j}}\left(N_{\mathrm{H}}, \Gamma_{i}, L_{\mathrm{X} i}, z_{i}\right) d \log N_{\mathrm{H}}} .
$$

The symbol $i$ represents each object, and $j$ represents each survey (only one in this case). Here, $A_{\mathrm{j}}$ gives the survey area for a count rate expected from a source with the absorption $N_{\mathrm{H}}$, photon index $\Gamma$, intrinsic luminosity $L_{X}$, and redshift $z$. The count rate is calculated through the detector response and luminosity distance by assuming the template spectra of AGNs presented in Section 4. The minimization is carried out on the MINUIT software package. In the ML fit, the $1 \sigma$ statistical error of a free parameter is derived as a deviation from the best-fit value when the $L^{\prime}$-value is increased by 1.0 from its minimum value.

Since the number of sources in this sample is limited, we only make $\psi_{43.75}^{0}$ as a free parameter, which represents the fraction of absorbed CTN AGNs in all CTN AGNs at $\log L_{\mathrm{X}}=$ 43.75. The other parameters on the absorption function are fixed as $\epsilon=1.7, \beta=0.24, a 1=0.48$ (the best-fit value from the whole sample, see Section 6), and $f_{\text {CTK }}=1.0$. We thus obtain $\psi_{43.75}^{0}=0.43 \pm 0.03$, which will be adopted in the following analysis. This is in perfect agreement with the result by Ueda et al. (2011) based on the MAXI sample, who obtain the absorption fraction at $\log L_{\mathrm{X}}=44$ of $\psi_{44}=0.37 \pm 0.05$, corresponding to $\psi_{43.75}^{0}=0.43 \pm 0.05$ with $\beta=0.24$.

The best-fit shape of the absorption function calculated at $L_{\mathrm{X}}=43.5$, the averaged value from the Swift/BAT sample, is plotted in the upper panel of Figure 4 by red lines. The data points in the same panel give a bias-corrected $N_{\mathrm{H}}$ distribution calculated in the following way. First, we make a normalized detection efficiency curve as a function of $N_{\mathrm{H}}$ that is proportional to $A_{\mathrm{j}}\left(N_{\mathrm{H}}, \Gamma_{i}, L_{\mathrm{X} i}, z_{i}\right)$ for each object. Then, the observed histogram of $N_{\mathrm{H}}$ is divided by the sum of the detection efficiency curves and is finally normalized to unity within the range between $\log N_{\mathrm{H}}=20-24$. A good agreement with the bestfit model is noted, justifying the choice of the fixed parameters of $\epsilon=1.7$ and $f_{\mathrm{CTK}}=1.0$. All parameters of the absorption function are summarized in Table 2.

\subsection{Evolution of Absorbed-AGN Fraction}

The dependence of the absorbed AGN fraction on redshift is studied in depth by Hasinger (2008), following previous works by La Franca et al. (2005), Ballantyne et al. (2006), and Treister \& Urry (2006), who all report a positive evolution in the sense that there are more obscured AGNs toward higher redshifts. Here, we pursue this issue by utilizing our new sample from the SXDS. It is not straightforward, however, to estimate a true intrinsic absorption fraction that is subject to detection biases as well as statistical errors in the measured $N_{\mathrm{H}}$ values, which become very large for faint AGNs detected in deep survey data, unlike the case of the local AGN sample analyzed in the

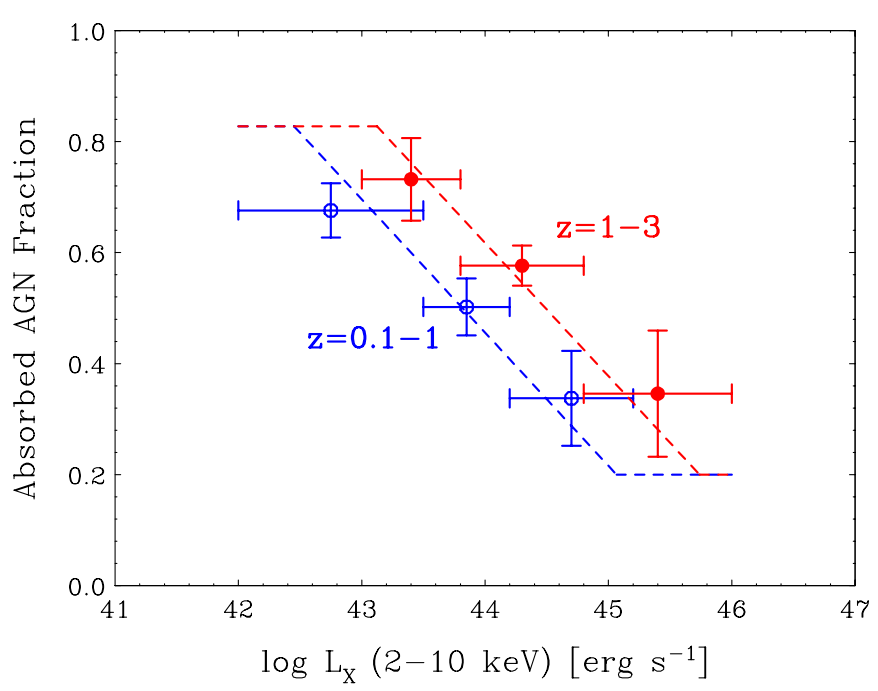

Figure 5. Absorbed fraction of AGNs plotted against luminosity at $z=0.1-1$ (blue) and $z=1-3$ (red) as determined from the Swift/BAT, AMSS, and SXDS (hard band) samples. The dashed lines represent the best-fit models calculated at the mean redshifts for the $z=0.1-1$ and $z=1-3$ samples.

(A color version of this figure is available in the online journal.)

previous subsection. To take into account the effects of statistical fluctuation in the hardness ratio, and hence that in $N_{\mathrm{H}}$ in the ML fit, we introduce the " $N_{\mathrm{H}}$ response matrix function," which gives the probability of finding an observed value of $N_{\mathrm{H}}$ from its true value for each object as described in Section 4.1 of U03. A similar approach is adopted in Hiroi et al. (2012), as well to estimate the absorption fraction at $z>3$ from the SXDS sample.

In this study, we utilize the samples of Swift/BAT, AMSS, and SXDS. To correctly calculate the $N_{\mathrm{H}}$ response matrix function, we need to have a hardness ratio and its statistical error. Although the absorptions are measured on the basis of spectral fitting in some samples, the resultant parameters are inevitably subject to non-negligible statistical errors much larger than those in the Swift/BAT sample. Unlike the simple method to estimate $N_{\mathrm{H}}$ only from the hardness ratio, it is difficult to quantify the effects of statistical fluctuation in individual spectral fit. We find that many faint Chandra sources have a very small number of photons in a given energy band. This makes the correction method more complicated because the statistics cannot be approximated by a Gaussian distribution. Thus, we decide to use only the AMSS sample and the SXDS hard-band sample, which have well defined the hardness ratio in two energy bands with enough photon statistics, in addition to the Swift/BAT sample. In this stage, for simplicity, we adopt the absorption, photon index, and luminosity calculated from the observed count rate and hardness ratio according to the procedure described in Section 2.8. The MAXI sample is not included to avoid duplication with the Swift/BAT AGNs. The parent sample thus consists of 751 AGNs with high identification completeness $(99 \%)$.

Using the list of $N_{\mathrm{H}}, \Gamma, L_{\mathrm{X}}$, and $z$ in the combined sample, we perform ML fitting of the absorption function on the basis of Equation (7). Since the main purpose is to investigate the luminosity and redshift dependence of absorption, we set $\beta=0$ and $a 1=0.0$ (i.e., constant) and make the $\psi_{43.75}^{0}$ parameter free by limiting to narrow ranges of $L_{\mathrm{X}}$ and $z$ (shown in Figure 5). Only the region of $\log N_{\mathrm{H}}=20-24$ is used in the ML fit, and thus the eight identified CTK AGNs in the $S$ wift/BAT sample are excluded. Since $N_{\mathrm{H}}$ is simply converted from the hardness ratio 
on the basis of a single absorbed continuum model for the AMSS and SXDS samples, it is assumed that there are no CTK AGNs in them, as mentioned in Section 2.8. This simplification would be justified because the fraction of CTK AGNs is expected to be small at the flux limits of the AMSS and the SXDS according to our population synthesis model (Section 7).

The results of the $\psi$ parameter are plotted in Figure 5. As noted, we confirm the strong anti-correlation between the absorption fraction and luminosity. In addition, a redshift dependence is clearly seen that the absorption fraction becomes larger at higher redshift by keeping the similar anti-correlation with luminosity. This is fully consistent with Hasinger (2008). These results support that the choice of $\beta=0.24$ that is constant against $z$ is appropriate. The $a 1$ parameter, representing the evolution with $z$, will be determined from the main analysis presented in Section 6, where the whole sample is utilized. The obtained best-fit curves calculated at the mean redshifts for the $z=0.1-1$ and $z=1-3$ samples are overplotted by dashed lines in the figure.

In the above analysis, we have assumed a single absorbed spectrum to estimate the column density for the AMSS and SXDS samples. In reality, it is known that the photon flux of an absorbed AGN reincrease toward the lowest energy because the absorber does not fully cover the nucleus, because the absorber is ionized, or because other soft components of different origins are present. According to the systematic spectral study of local AGNs detected in the Swift/BAT survey (Winter et al. 2009a; Ichikawa et al. 2012), in most cases, the X-ray spectra of absorbed AGNs can be well represented by the partial covering model, where a fraction of $f_{\mathrm{c}}$ of the total continuum is subject to absorption. A majority of absorbed AGNs have covering fractions of $f_{\mathrm{c}} \gtrsim 0.98$, which can be explained by the leakage of the scattered component from outside the torus (see Section 4), while $\sim 15 \%$ of the total AGNs show smaller covering fractions, with a medium of $f_{\mathrm{c}} \approx 0.95$, most probably due to the complex nature of the absorber or the presence of other components. To evaluate the possible systematic errors, we statistically take into account the distribution of these complex spectra in calculating the " $N_{\mathrm{H}}$ response matrix function" and repeat the analysis. We confirm that systematic errors in the absorbed AGN fractions are much smaller than the statistical errors at any luminosity and redshift regions, and hence our conclusions are robust.

\section{TEMPLATE MODEL SPECTRA OF AGNs}

In our main analysis, described in Section 6, we simultaneously treat the results from the surveys performed in different energy bands spanning from $0.5 \mathrm{keV}$ to $195 \mathrm{keV}$. Thus, it is critical to make consistent analysis on the basis of adequate assumptions on the broadband X-ray spectra of AGNs. In this section, we describe "template spectra" of AGNs adopted in this paper. They are based on extensive studies of broadband spectra of nearby AGNs in the literature. As for the intrinsic continuum spectrum, we adopt a cutoff power law with $E_{\mathrm{c}}=300 \mathrm{keV}$, an averaged value of bright Seyfert galaxies in the local universe reported by Dadina (2008). To achieve a physically consistent picture, two reflection components from optically thick matter are considered, one from the accretion disk and the other from the torus. The solar abundances are assumed in both models. We also add an unabsorbed scattered component from the surrounding gas located outside the torus. For simplicity, the spectrum is assumed to be a pure Compton scattered one of the continuum without any emission lines, by taking into account the Klein-Nishina cross-section. Its intensity is proportional to the opening solid angle of the torus, which is normalized to be $1 \%$ when the half sky $(\Omega=2 \pi)$ is covered by it (Eguchi et al. 2011).

We model the reflection component from the accretion disk with the pexrav code (Magdziarz \& Zdziarski 1995) by assuming that the disk is not ionized. The parameters are the inclination angle of the line of sight with respect to the normal axis of the disk, $\theta_{\text {inc }}$, and the reflection strength $R_{\text {disk }}$, for which we adopt $R_{\text {disk }}=0.5$ as a default value. This value is chosen to roughly explain an averaged reflection strength found in local Seyfert galaxies, $R_{\text {tot }} \sim 1$ (e.g., Zdziarski et al. 1995; Dadina 2008), after the contribution from the torus reflection component is added. Its possible dependences on $L_{\mathrm{X}}$ and on $z$ are ignored, for which no consensus has been established yet. The inclination $\theta_{\text {inc }}$ is determined separately for type- 1 and type- 2 AGNs as a function of $L_{\mathrm{X}}$ and $z$ according to the description below.

To reproduce realistic spectra from AGN tori, even in CTK cases, we adopt the numerical model calculated by Brightman \& Nandra (2011a) based on Monte Carlo simulations, where the absorbing torus is approximated by a uniform sphere with bipolar conical openings, rather than donut-shaped openings. The model parameters are the photon index $\Gamma$, the column density $N_{\mathrm{H}}^{\text {torus }}$, the opening angle $\theta_{\mathrm{oa}}$ ( or $\theta_{\text {tor }}$ ), and the inclination angle $\theta_{\mathrm{i}}$ (see Figure 1 of Brightman \& Nandra 2011a). The spectrum consists of the transmitted emission (unabsorbed when $\theta_{\mathrm{i}}<\theta_{\text {oa }}$ and vice versa), its reflected spectra from the torus, including fluorescence lines from abundant metals (such as iron, nickel, etc.). Self-absorption of the reprocessed emission by the torus itself is properly taken into account for a given geometry.

Throughout our work, we assume the "luminosity- and redshift-dependent unified scheme" of AGNs; the absorbed fraction of AGNs is simply determined by the covering factor of the torus whose geometry depends on both $L_{X}$ and $z$. The torus opening angle can be then related to the fraction of total absorbed AGNs (including CTK AGNs) among all AGNs, $\psi^{\prime}\left(L_{\mathrm{X}}, z\right)$, as

$$
\begin{aligned}
\cos \left(\theta_{\mathrm{oa}}\right) & =\psi^{\prime}\left(L_{\mathrm{X}}, z\right) \\
& =\frac{\left(1+f_{\mathrm{CTK}}\right) \psi\left(L_{\mathrm{X}}, z\right)}{1+f_{\mathrm{CTK}} \psi\left(L_{\mathrm{X}}, z\right)} .
\end{aligned}
$$

We recall that the absorbed AGNs are defined as those with $\log N_{\mathrm{H}} \geqslant 22$. This is based on the idea that absorptions smaller than $\log N_{\mathrm{H}}=22$ are not originated from the torus but most probably from interstellar medium in the host galaxy (see, e.g., Fukazawa et al. 2011). Hence, we multiply the absorption to all of the spectral components for type-1 AGNs (i.e., with $\log N_{\mathrm{H}}<22$ ), while we ignore other origins than the tori for the absorptions in type-2 AGNs. In this formulation, we assume that CTK AGNs are just an extension of CTN AGNs with the same $L_{\mathrm{X}}$ and $z$ toward larger column densities. Using a type-1 AGN sample in the local universe, Ricci et al. (2013) find that the luminosity-dependent unified scheme can explain the socalled X-ray Baldwin effect (Iwasawa \& Taniguchi 1993), the anti-correlation between the equivalent width of the iron-K line and X-ray luminosity, although the definition of the absorbed fraction is slightly different from ours; when they refer to the result by Hasinger (2008), AGNs with $\log N_{\mathrm{H}} \geqslant 21.5$ are counted as absorbed ones by neglecting CTK AGNs.

Once $\theta_{\mathrm{oa}}$ is known, we can calculate a solid-angle weighted average of the inclination angle separately for type 1 and type 2 AGNs:

$$
\theta_{\text {inc }}= \begin{cases}\left.\arccos \left(1-\left(1-\cos \theta_{\mathrm{oa}}\right) / 2\right)\right) & (\text { for type-1) } \\ \left.\arccos \left(\cos \theta_{\mathrm{oa}} / 2\right)\right) & (\text { for type-2) }\end{cases}
$$



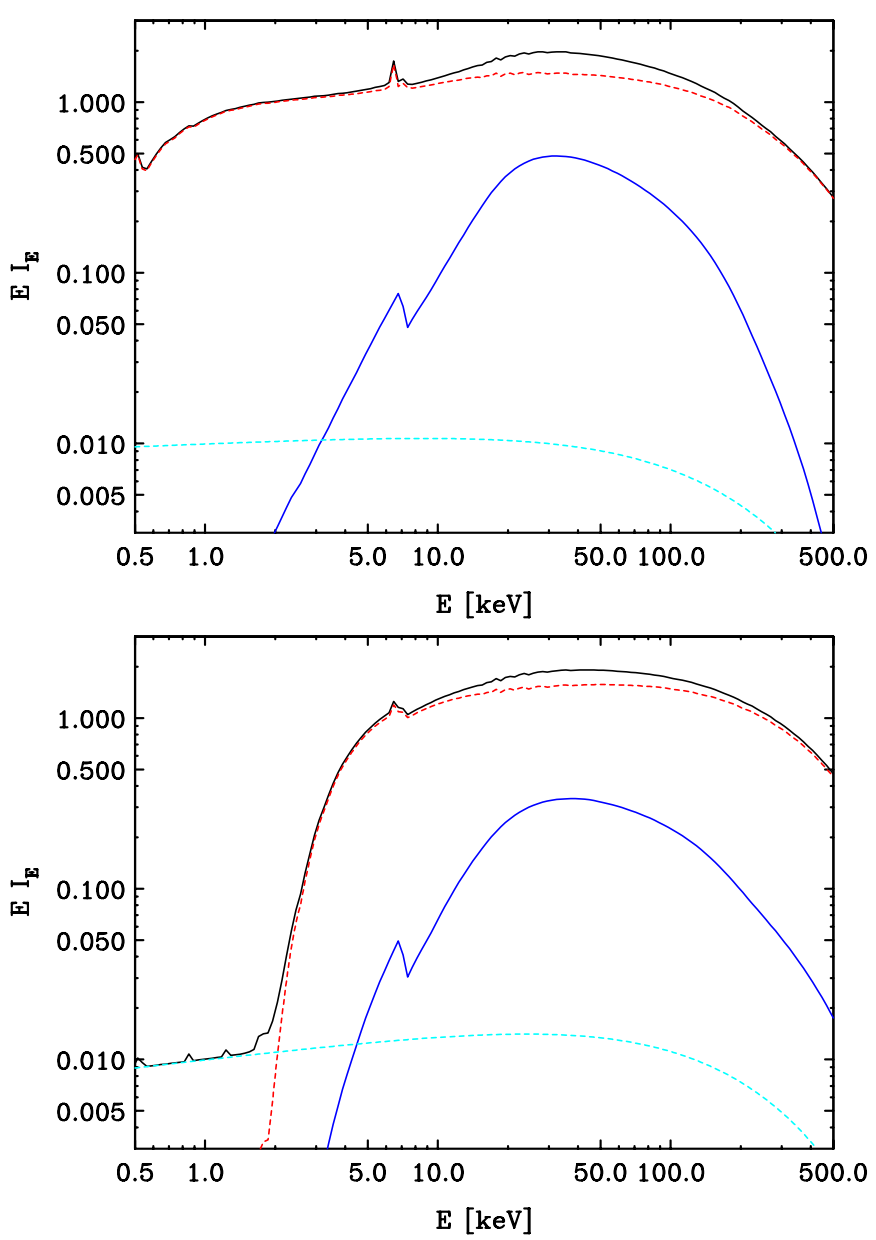

Figure 6. Examples of the template broadband X-ray spectra (in $E I(E)$, where $E$ is energy and $I(E)$ is the energy flux) for AGNs with $\log N_{\mathrm{H}}=21$ and $\Gamma=1.94$ (top) and $\log N_{\mathrm{H}}=23$ and $\Gamma=1.84$ (bottom). Units are arbitrary. The black curve denotes the total spectrum, the red curve the transmitted continuum plus its reflection component from the torus, the blue curve the reflection component from the accretion disk, and the cyan curve the scattered component.

(A color version of this figure is available in the online journal.)

According to the torus geometry in the Brightman \& Nandra (2011a) model, the torus column density $N_{\mathrm{H}}^{\text {torus }}$ is taken to be the same as the line-of-sight column density $N_{\mathrm{H}}$ in type- 2 AGNs. For type- 1 AGNs, we adopt $\log N_{\mathrm{H}}^{\text {torus }}=24$ as an average value to reflect our assumption that the number of CTK AGNs is the same as CTN absorbed ones (i.e., $f_{\text {CTK }}=1.0$ ).

Figure 6 (top) and (bottom) plot the template model spectra in the $0.5-500 \mathrm{keV}$ band for an AGN with $\log N_{\mathrm{H}}=21$ and 23 , respectively. Here, we adopt $\Gamma=1.94$ for the former and $\Gamma=1.84$ for the latter (see Section 5). The disk reflection and scattered components are shown separately. Figure 7 shows those for $\log N_{\mathrm{H}}=20.5,21.5,22.5,23.5,24.5$, and 25.5. For easy comparison, we adopt $\Gamma=1.9$ for all the spectra in this figure. The suppression of the hard X-ray flux in heavily CTK AGNs is noted. In both figures, $\theta_{\mathrm{oa}}=60^{\circ}$ is assumed.

\section{PHOTON INDEX DISTRIBUTION}

To estimate the intrinsic photon index $(\Gamma)$ distribution of AGNs in the local universe, we analyze the Swift/BAT spectra in the 14-195 keV band averaged for 58 months, which are available for all AGNs in the Swift/BAT 9 month catalog. This energy band is suitable to estimate the $\Gamma$ value of an individual CTN AGN as a first order approximation without invoking

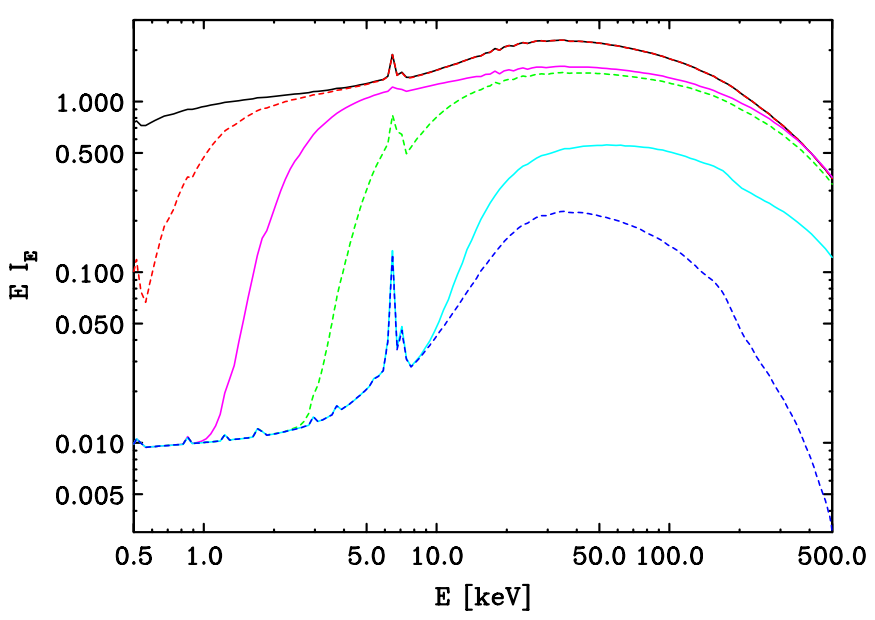

Figure 7. Model spectra of AGNs with different absorptions (from top to bottom, $\left.\log N_{\mathrm{H}}=20.5,21.5,22.5,23.5,24.5,25.5\right)$. Units are arbitrary in $E I(E)$. A same photon index $(\Gamma=1.9)$ and a normalization are assumed for the intrinsic cut-off power-law continuum in all spectra.

(A color version of this figure is available in the online journal.)

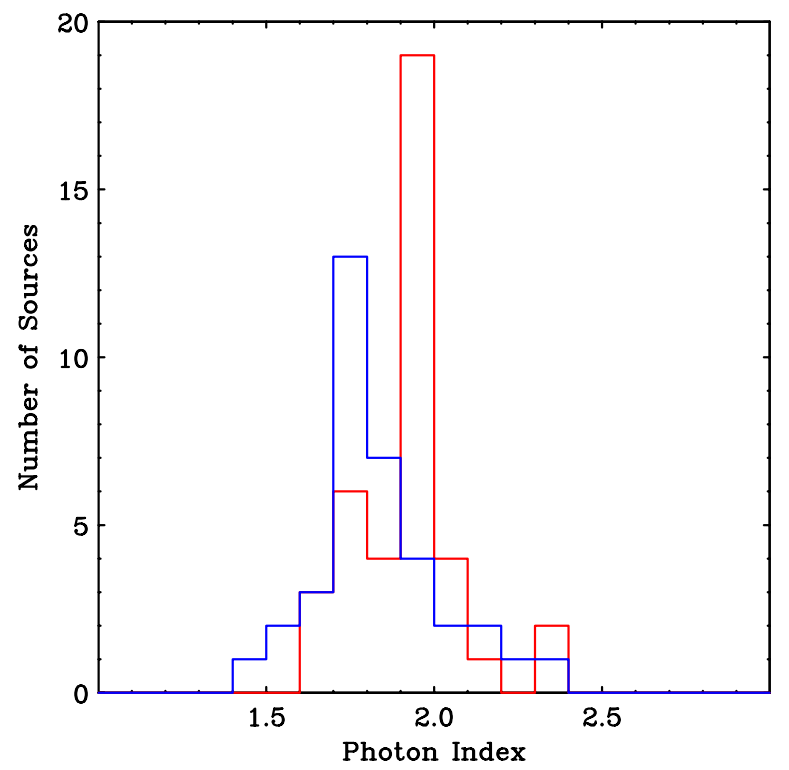

Figure 8. Distribution of photon index for type-1 (red) and type-2 (blue) AGNs as determined from the Swift/BAT survey.

(A color version of this figure is available in the online journal.)

complex, source-dependent spectral analysis covering the full 0.5-195 keV band, which is beyond the scope of this paper. We systematically perform spectral fitting to the $14-195 \mathrm{keV}$ spectra of the Swift/BAT sample with the "template spectra." The parameters of the reflection and scattered components are fixed according to our best estimate of the $\psi\left(L_{\mathrm{X}}, z\right)$ values (Section 3), and only the photon index and overall normalization are free parameters.

Figure 8 displays the histograms of the best-fit photon index, plotted separately for type-1 (red) and type-2 AGNs (blue). Here, we exclude the results for four objects out of 79 CTN AGNs in the original sample for which the goodness of the fit is found to be poor. Since it composes only a minor fraction, we regard the rest of the sample as a representative one. As noted from the figure, we confirm the trend reported previously (e.g., Zdziarski et al. 2000; Tueller et al. 2008; Burlon et al. 2011) that the average slope of type- 1 AGNs is larger than that 
Table 3

Parameters of Photon Index Function

\begin{tabular}{lccc}
\hline$\overline{\overline{\Gamma_{1}}}$ & $\Delta \Gamma_{1}$ & $\overline{\Gamma_{2}}$ & $\Delta \Gamma_{2}$ \\
\hline $1.94 \pm 0.03$ & $0.09 \pm 0.05$ & $1.84 \pm 0.04$ & $0.15 \pm 0.06$ \\
\hline
\end{tabular}

Notes. Attached errors ( $1 \sigma)$ are based on the Swift/BAT 9 month sample. These are fixed at the best-fit values when performing ML fit to the whole sample (Section 6).

of type-2 AGNs, even after properly taking into account the reflection components in the spectra. This can also explain the suggestion by Ueda et al. (2011) that the averaged photon index in the 4-195 keV band is larger in a high-luminosity range where type-1 AGNs dominate their sample. From these histograms, we obtain the average and standard deviation (with their $1 \sigma$ errors) of $\overline{\Gamma_{1}}=1.94 \pm 0.03$ and $\Delta \Gamma_{1}=0.09 \pm 0.05$ for type- 1 AGNs, and $\overline{\Gamma_{2}}=1.84 \pm 0.04$ and $\Delta \Gamma_{2}=0.15 \pm 0.06$ for type- 2 AGNs, as summarized in Table 3. Here, $\Delta \Gamma_{1,2}$ presents the intrinsic scatter of the photon index after subtracting that caused by the statistical errors in the spectral fits.

To quantitatively incorporate the intrinsic scatter of photon index, we introduce the "photon index function," $g\left(N_{\mathrm{H}}, L_{\mathrm{X}}, z ; \Gamma\right)$, similar to the absorption function, which gives the probability of finding $\Gamma$ per unit $\Gamma$ space from an AGN with $N_{\mathrm{H}}$ and $L_{\mathrm{X}}$ located at $z$. Specifically, we model it by a normalized Gaussian function as

$$
g\left(L_{\mathrm{X}}, z, N_{\mathrm{H}} ; \Gamma\right)=\frac{1}{\sqrt{2 \pi} \Delta \Gamma_{1,2}} \exp \left[-\frac{\left(\Gamma-\overline{\Gamma_{1,2}}\right)^{2}}{2\left(\Delta \Gamma_{1,2}\right)^{2}}\right],
$$

where $\overline{\Gamma_{1}}=1.94$ and $\Delta \Gamma_{1}=0.09$ for $\log N_{\mathrm{H}}<22$, and $\overline{\Gamma_{2}}=1.84$ and $\Delta \Gamma_{2}=0.15$ for $\log N_{\mathrm{H}} \geqslant 22$. In our paper, we ignore the $L_{\mathrm{X}}$ and $z$ dependences, which have not been established yet. The effects of changing the $\overline{\Gamma_{1,2}}$ or $\Delta \Gamma_{1,2}$ parameters on our final results will be examined in Section 7.2.

\section{LUMINOSITY FUNCTION}

\subsection{Analysis Method}

In this section, we describe the main part of analysis where the XLF is determined by performing an ML fit to our sample at $z=0.002-5.0$. We define the XLF of CTN AGNs so that

$$
\frac{d \Phi_{\mathrm{X}}^{\mathrm{CTN}}\left(L_{\mathrm{X}}, z\right)}{d \log L_{\mathrm{X}}}
$$

represents the number density per unit co-moving volume per $\log L_{\mathrm{X}}$ as a function of $L_{\mathrm{X}}$ and $z$ in units of $\mathrm{Mpc}^{-3} \mathrm{dex}^{-1}$. The ML fit to unbinned data is a standard method to determine the model parameters when the sample size is limited. Unlike in many previous works, however, we do not use the list of $L_{X}$ and $z$ as the input data. This is because, as already mentioned in Section 2.8, we cannot avoid uncertainties in the determination of $L_{X}$ for which an accurate measurement of absorption is required, while spectral information is limited due to poor statistics in faint sources. In particular, when we expand the $N_{\mathrm{H}}$ region of interest above $\log N_{\mathrm{H}}>24$ to include CTK AGNs, the hardness ratio does not uniquely correspond to a single $N_{\mathrm{H}}$ value because of the complexity of the spectrum, producing additional systematic errors to determine $L_{\mathrm{X}}$.

Thus, we develop a new analysis method to utilize the list of the count rate $C_{\mathrm{X}}$ and $z$ obtained in each survey, the most basic observational quantities without any corrections. This approach is based on a "forward method," where comparison between the prediction and observation is made at the final stage. Namely, we search for a set of parameters of the XLF and absorption function that best reproduce the count rate versus $z$ distributions of all surveys used in the analysis. The merit is that we can properly take into account any complex X-ray spectra in the analysis, including CTK AGNs. Another merit is that we can treat surveys of the same field (including all sky surveys) in different energy bands as independent data of one another, enabling us to utilize all samples without worrying about the overlap of objects detected in multiple surveys.

Specifically, we define a likelihood estimator as

$$
L=-2 \sum_{i} \ln \frac{\iiint N_{j}\left(N_{\mathrm{H}}, \Gamma, \hat{L}_{\mathrm{X}}, z\right) d \log N_{\mathrm{H}} d \Gamma d z}{\sum_{j} \iiint \int N_{j}\left(N_{\mathrm{H}}, \Gamma, L_{\mathrm{X}}, z\right) d \log N_{\mathrm{H}} d \Gamma d \log L_{\mathrm{X}} d z},
$$

where

$$
\hat{L}_{\mathrm{X}}=4 \pi d_{\mathrm{L}}^{2}(z) a_{j}\left(N_{\mathrm{H}}, \Gamma, z\right) C_{\mathrm{X} i}
$$

in the integrand of the numerator. The suffixes $i$ and $j$ represent each independent detection and survey, respectively. Here, $d_{\mathrm{L}}(z)$ is the luminosity distance, $a_{j}\left(N_{\mathrm{H}}, \Gamma, z\right)$ is the conversion factor from the count rate in the $j$ th survey into the de-absorbed flux in the observer's frame $2 /(1+z)-10 /(1+z) \mathrm{keV}$ band, and $C_{\mathrm{X} i}$ is the observed count rate of the $i$ th detection. The term $N_{j}\left(N_{\mathrm{H}}, \Gamma, L_{\mathrm{X}}, z\right)$ represents the expected number from the $j$ th survey calculated as

$$
\begin{aligned}
& N_{j}\left(N_{\mathrm{H}}, \Gamma, L_{\mathrm{X}}, z\right)=f\left(L_{\mathrm{X}}, z ; N_{\mathrm{H}}\right) g\left(L_{\mathrm{X}}, z, N_{\mathrm{H}} ; \Gamma\right) \\
& \quad \times \frac{d \Phi_{\mathrm{X}}^{\mathrm{CTN}}\left(L_{\mathrm{X}}, z\right)}{d \log L_{\mathrm{X}}} d_{\mathrm{A}}(z)^{2}(1+z)^{3} c \frac{d \tau}{d z}(z) A_{j}\left(N_{\mathrm{H}}, \Gamma, L_{\mathrm{X}}, z\right),
\end{aligned}
$$

where $d_{\mathrm{A}}(z)$ is the angular distance, $d \tau / d z$ is the differential look back time, and $A_{j}\left(N_{\mathrm{H}}, \Gamma, L_{\mathrm{X}}, z\right)$ is the area of the $j$ th survey expected from an AGN with $N_{\mathrm{H}}, \Gamma, L_{\mathrm{X}}$, and $z$.

In principle, it is possible to simultaneously constrain the XLF, absorption function $f\left(L_{\mathrm{X}}, z ; N_{\mathrm{H}}\right)$ and photon index function $g\left(L_{\mathrm{X}}, z, N_{\mathrm{H}} ; \Gamma\right)$ through an ML fit. In practice, however, to avoid strong parameter coupling we only make the index of the evolution factor $a 1$ in the absorption function (Equation (4)) as a free parameter, besides those in the XLF. In our baseline model, the other parameters of the absorption function and photon index function are all fixed at the values presented in Sections 3 and 5, respectively. Since this ML fit does not constrain the normalization of the XLF, we determine it so that the expected number of the total detections agrees with the observed one, $N_{\text {tot }}=4039$, and basically estimate the uncertainty from its Poisson error (but see below). In ML fits, the minimized value of the likelihood estimator itself cannot be utilized to evaluate the absolute goodness of the fit. Thus, we verify it by comparing the flux and redshift distribution between the model prediction and observation on the basis of a $\chi^{2}$ test.

In a normal ML fit performed to a completely independent data set (like the analysis presented in Section 3.2), the $1 \sigma$ error for a single parameter is defined as the deviation from the best fit when the $L$-value is increased by $\Delta-L=1.0$ from its minimum value. In our case, however, we utilize multipleband data from the same objects (i.e., at common redshifts) for a significant fraction of the sample, which would work to underestimate the true errors in the XLF parameters. Hence, here we conservatively estimate their $1 \sigma$ errors by adopting 
$\Delta-L=2.0$ instead of $\Delta-L=1.0$, to take into account the "double counting" effects. For the same reason, we estimate the relative uncertainty in the normalization of the XLF as $1 / \sqrt{N_{\text {tot }} / 2}$ instead of $1 / \sqrt{N_{\text {tot }}}$.

In our analysis, we neglect the effects of AGN variability in determination of the XLF. Many X-ray surveys utilized in our study are, however, based on observations with a typical exposure of $\sim 1$ day, except for the Swift/BAT and MAXI surveys and very deep ones like the CDFN and CDFS. This means that we measure an instantaneous flux (or luminosity) of an AGN, which may well be different from its "true" flux averaged over a much longer period. For instance, Paolillo et al. (2004) report that most of AGNs in the CDFS possess intrinsic $\mathrm{X}$-ray variability on timescales ranging from a day to a year. They find that the fractional variability is $\lesssim 0.2$ for $90 \%$ of the AGN. To check the possible systematic effects, we thus perform an ML fit with the same XLF model as described in Section 6.2 by taking into account variability of each AGN in Equation (12), except for the Swift/BAT, MAXI, CDFN, and CDFS samples. Here, the distribution of the observed flux relative to the intrinsic one is assumed to be a Gaussian with a standard deviation of 0.2 , which is adopted as the maximum value regardless of the luminosity. The result verifies that the best-fit XLF parameters are not affected by the time variability over the statistical uncertainties.

\subsection{Results}

We model the luminosity function in the local universe by a smoothly connected double power-law model that has slopes $\gamma_{1}$ and $\gamma_{2}$ below and above the break luminosity $L_{*}$, respectively:

$$
\frac{d \Phi_{\mathrm{X}}^{\mathrm{CTN}}\left(L_{\mathrm{X}}, z=0\right)}{d \log L_{\mathrm{X}}}=A\left[\left(L_{\mathrm{X}} / L_{*}\right)^{\gamma_{1}}+\left(L_{\mathrm{X}} / L_{*}\right)^{\gamma_{2}}\right]^{-1} \text {. }
$$

Many previous works, based on a sample covering a sufficiently wide $L_{X}$ and $z$ range, have revealed that the evolution of the $\mathrm{XLF}$ is more complex than that approximated by a simple model like the pure density evolution or the pure luminosity evolution. Here, we adopt the LDDE, which is found to give a good representation of the XLF of AGNs in a number of studies based on hard X-ray ( $>2 \mathrm{keV}$ ) (Ueda et al. 2003; Silverman et al. 2008; Ebrero et al. 2009; Yencho et al. 2009) and soft $\mathrm{X}$-ray $(<2 \mathrm{keV}$ ) selected samples (Miyaji et al. 2000; Hasinger et al. 2005).

We basically follow the formulation of the XLF in U03 with a few additional modifications. The XLF at a given $z$ is calculated by multiplying a luminosity-dependent evolution factor $e\left(z, L_{\mathrm{X}}\right)$ to the local one:

$$
\frac{d \Phi_{\mathrm{X}}^{\mathrm{CTN}}\left(L_{\mathrm{X}}, z\right)}{d \log L_{\mathrm{X}}}=\frac{d \Phi_{\mathrm{X}}^{\mathrm{CTN}}\left(L_{\mathrm{X}}, 0\right)}{d \log L_{\mathrm{X}}} e\left(z, L_{\mathrm{X}}\right) .
$$

Recent studies, based on large area surveys like COSMOS and SXDS, with high completeness have established a decay of the comoving number density of luminous AGNs with $L_{\mathrm{X}} \gtrsim 44$ toward higher redshift above $z \gtrsim 3$ (Brusa et al. 2009; Civano et al. 2011; Hiroi et al. 2012). The similar trend was suggested in a previous work by Silverman et al. (2005) using a Chandra serendipitous survey called CHAMP, where the completeness correction was made in the X-ray and optical flux plane. Hence, we take into account the decline in the evolution factor by introducing another (luminosity-dependent) cut-off redshift above which the decline of $d \Phi_{\mathrm{X}}^{\mathrm{CTN}}\left(L_{\mathrm{X}}, z\right) / d \log L_{\mathrm{X}}$ with $z$ starts to appear.
The evolution factor as a function of $z$ and $L_{X}$ is thus represented as

$$
e\left(z, L_{\mathrm{X}}\right)= \begin{cases}(1+z)^{p 1} & {\left[z \leqslant z_{c 1}\left(L_{\mathrm{X}}\right)\right]} \\ \left(1+z_{c 1}\right)^{p 1}\left(\frac{1+z}{1+z_{c 1}}\right)^{p 2} & {\left[z_{c 1}\left(L_{\mathrm{X}}\right)<z \leqslant z_{c 2}\right]} \\ \left(1+z_{c 1}\right)^{p 1}\left(\frac{1+z_{c 2}}{1+z_{c 1}}\right)^{p 2}\left(\frac{1+z}{1+z_{c 2}}\right)^{p 3} & {\left[z>z_{c 2}\right] .}\end{cases}
$$

Here, $z_{\mathrm{c} 1}$ and $z_{\mathrm{c} 1}$ represent two cut-off redshifts where the evolution index changes from $p 1$ to $p 2$ and from $p 2$ to $p 3$, respectively. We adopt $p 2=-1.5$, the same value as adopted in U03, and $p 3=-6.2$, based on the result by Hiroi et al. (2012). Following H05, we consider the luminosity dependence for the $p 1$ parameter as

$$
p 1\left(L_{\mathrm{X}}\right)=p 1^{*}+\beta_{1}\left(\log L_{\mathrm{X}}-\log L_{\mathrm{p}}\right),
$$

where we set $\log L_{\mathrm{p}}=44$.

Both cutoff redshifts are given by power-law functions of $L_{\mathrm{X}}$ with indices of $\alpha 1$ and $\alpha 2$ below luminosity thresholds of $L_{\mathrm{a} 1}$ and $L_{\mathrm{a} 2}$, respectively:

$$
z_{\mathrm{c} 1}\left(L_{\mathrm{X}}\right)= \begin{cases}z_{\mathrm{c} 1}^{*}\left(L_{\mathrm{X}} / L_{\mathrm{a} 1}\right)^{\alpha 1} & {\left[L_{\mathrm{X}} \leqslant L_{\mathrm{a} 1}\right]} \\ z_{\mathrm{c} 1}^{*} & {\left[L_{\mathrm{X}}>L_{\mathrm{a} 1}\right]}\end{cases}
$$

and

$$
z_{\mathrm{c} 2}\left(L_{\mathrm{X}}\right)= \begin{cases}z_{\mathrm{c} 2}^{*}\left(L_{\mathrm{X}} / L_{\mathrm{a} 2}\right)^{\alpha 2} & {\left[L_{\mathrm{X}} \leqslant L_{\mathrm{a} 2}\right]} \\ z_{\mathrm{c} 2}^{*} & {\left[L_{\mathrm{X}}>L_{\mathrm{a} 2}\right] .}\end{cases}
$$

We fix $z_{\mathrm{c} 2}^{*}=3.0, \log L_{\mathrm{a} 2}=44$, and $\alpha 2=-0.1$, which well represent our XLF at $z>3$ and are also consistent with the results by Fiore et al. (2012), based on multiwavelength studies in the CDFS field.

Adopting the LDDE model for the XLF along with the absorption and photon index functions described in Sections 3 and 5, we perform an ML fit to the whole sample consisting of 4039 detections. The evolution index $a 1$ in the absorption function and all parameters of the XLF, except for those mentioned as fixed above, are left to be free parameters. The best-fit parameters and the $1 \sigma$ errors are summarized in Tables 2 (a1) and 4 (XLF). To verify the absolute goodness of the fit, we calculate the two-dimensional histogram of flux and redshift predicted by the best-fit model. The count rates in each survey are converted to the $2-10 \mathrm{keV}$ flux by assuming a power-law index of 1.4 so that we can combine the results from the multiple surveys. The histogram has 10 and 17 logarithmic bins in the flux range between $10^{-9}-10^{-17}$ and the redshift range between $0.002-5.0$, respectively. To compare it with the observed histogram made in the same way, the $\chi^{2}$ value between the two histograms is calculated by adopting the $1 \sigma$ error of $1+\sqrt{N+0.75}$ in each bin of the observed histogram, where $N$ is the number of sources (Gehrels 1986). We obtain $\chi^{2}=102.7$ with a degree of freedom (dof) of 114, indicating that the model is acceptable. Figure 9 (left) and (right) show the projected histograms onto the flux and redshift axes, respectively, together with the model predictions (curve). Good agreements between the data and model are seen, although there is a peak feature in the observed redshift distribution around $z \approx 1.5$, related to the large-scale structure in the SXDS field (M. Akiyama et al. 2014 , in preparation).

Figure 10 displays the best-fit XLFs of CTN AGNs, $d \Phi_{\mathrm{X}}^{\mathrm{CTN}}\left(L_{\mathrm{X}}, z=0\right) / d \log L_{\mathrm{X}}$, in 12 different redshift bins covering from $z=0.002$ to $z=5.0$. The shape of the XLF at 

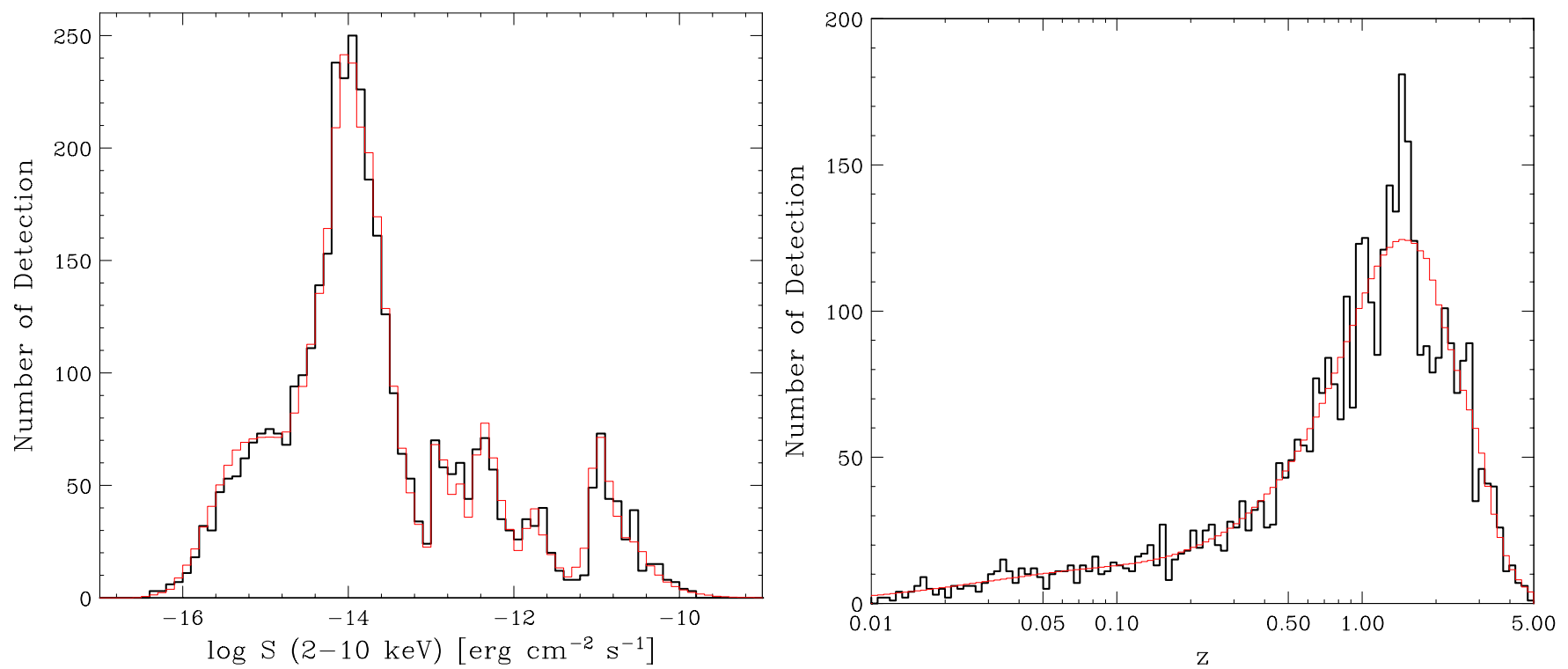

Figure 9. Observed histograms (thick, black) of flux (left) and redshift (right) of our sample compared with model predictions (thin, red).

(A color version of this figure is available in the online journal.)

Table 4

Best-fit Parameters of AGN Luminosity Functions

\begin{tabular}{|c|c|c|c|c|c|c|c|c|c|}
\hline Band & $A^{\mathrm{a}}$ & $\log L_{*}$ & $\gamma_{1}$ & $\gamma_{2}$ & $p 1^{*}$ & $\beta_{1}$ & $z_{\mathrm{c} 1}^{*}$ & $\log L_{\mathrm{a} 1}$ & $\alpha_{1}$ \\
\hline $2-10 \mathrm{keV}$ & $2.91 \pm 0.07$ & $43.97 \pm 0.06$ & $0.96 \pm 0.04$ & $2.71 \pm 0.09$ & $4.78 \pm 0.16$ & $0.84 \pm 0.18$ & $1.86 \pm 0.07$ & $44.61 \pm 0.07$ & $0.29 \pm 0.02$ \\
\hline Bolometric & $3.26 \pm 0.08$ & $45.48 \pm 0.09$ & $0.89 \pm 0.03$ & $2.13 \pm 0.07$ & $5.33 \pm 0.15$ & $0.46 \pm 0.15$ & $1.86^{\mathrm{b}}$ & $46.20 \pm 0.04$ & $0.29^{\mathrm{b}}$ \\
\hline
\end{tabular}

Notes. Errors are $1 \sigma$ for a single parameter. Only free parameters are listed for the XLF; we fix $p 2=-1.5, p 3=-6.2, z_{\mathrm{c} 2}^{*}=3.0, \log L_{\mathrm{p}}=\log L_{\mathrm{a} 2}=44(45.67)$ for the XLF (BLF), and $\alpha 2=-0.1$ (see Section 6).

${ }^{\text {a }}$ In units of $\left[10^{-6} h_{70}^{3} \mathrm{Mpc}^{-3}\right]$.

${ }^{\mathrm{b}}$ Fixed.

the central redshift of each bin is represented by the curves. The data points are calculated on the basis of the " $N^{\mathrm{obs}} / N^{\mathrm{mdl}}$ method" (Miyaji et al. 2001); for a given luminosity bin, we plot the model at the logarithmic center of $L_{X}$ multiplied by the ratio between the number of observed sources and that of the model prediction. Here, we utilize the $L_{\mathrm{X}}$ value assigned to each object according to the procedures described in Section 2.8. Thus, the plotted data should be regarded as an approximation by considering the uncertainties in calculating $L_{X}$, in particular, for CTK AGNs. This would become an issue only for faintest AGNs detected in the hard band, $\sim 10 \%-20 \%$ of which could be CTK AGNs at fluxes of $S=10^{-15} \mathrm{to}^{-16} \mathrm{erg} \mathrm{cm}^{-2} \mathrm{~s}^{-1}(2-10 \mathrm{keV})$ according to our best-fit model (see Section 7.1). The data points are independently calculated from the hard-band $(>2 \mathrm{keV})$ and soft-band $(<2 \mathrm{keV})$ samples, which are marked by filled (blue) and open circles (red), respectively. Here, the MAXI sample is not included due to its significant overlap with the Swift/BAT sources. The error bars reflect the relative Poissonian $1 \sigma$ errors in the observed number of sources based on the formula of Gehrels (1986). The arrows denote the 90\% confidence upper limits when no object is found in that luminosity bin. To show the redshift dependence of the XLF, we plot the best-fit model computed at different redshifts in Figure 11.

In Figure $10(z=3.0-4.0$ and $z=4.0-5.0)$, we also plot the luminosity function derived by Fiore et al. (2012). They adopt a fainter flux limit than that in Xue et al. (2011) by utilizing the positional information of $z>3$ galaxies based on optical and mid-infrared catalogs. Our best-fit XLF is in good agreement with their results; the maximum deviation of the data points is $<2 \sigma$ statistical error. Note that Vito et al. (2013) analyze the $z>3$ AGNs in the CDFS by adopting rather conservative selection criteria. The $\log N-\log S$ relation of their sample is smaller than that of Fiore et al. (2012) by a factor of $\sim 2$. The discrepancy could be explained by incompleteness. The same problem could be present in our sample, too. However, assuming the extreme case that all of the unidentified AGNs detected in the soft band are $z>3$ AGNs, we find that the XLF normalization at $z>3$ becomes only 1.5 times larger than the present data points in average, which is still consistent with the best-fit model within the errors.

Figure 12 plots the co-moving space number density of CTN AGNs as a function of redshift integrated in different luminosity bins, $\log L_{X}=42-43,43-44,44-45$, and 45-47. The curves show that of the best-fit model, while the data points are based on the " $N$ obs $/ N^{\text {mdl }}$ method" as explained above. In this figure, to ensure complete independence of the plotted data, we utilize either the hard-band- or soft-band-selected sample to calculate the data points in each redshift and luminosity bin. Specifically, we adopt the hard-band samples (without the MAXI one) in the region of $z<2$ and $\log L_{\mathrm{X}}<44$, and the soft-band samples for the rest. This is because at higher redshifts, soft-band surveys become more efficient even for obscured AGNs, thanks to the $K$-correction effect. Also, at large $L_{\mathrm{X}}$ ranges, the majority of AGNs are unobscured populations (Section 3), for which wide area surveys with ROSAT provide a large number of sources.

From Figure 12, one can clearly confirm the global "downsizing" evolution, where more luminous AGNs have their number density peak at higher redshifts compared with less luminous ones. We note, however, that when we only focus attention on the high-redshift range of $z \gtrsim 3$, our LDDE model with 

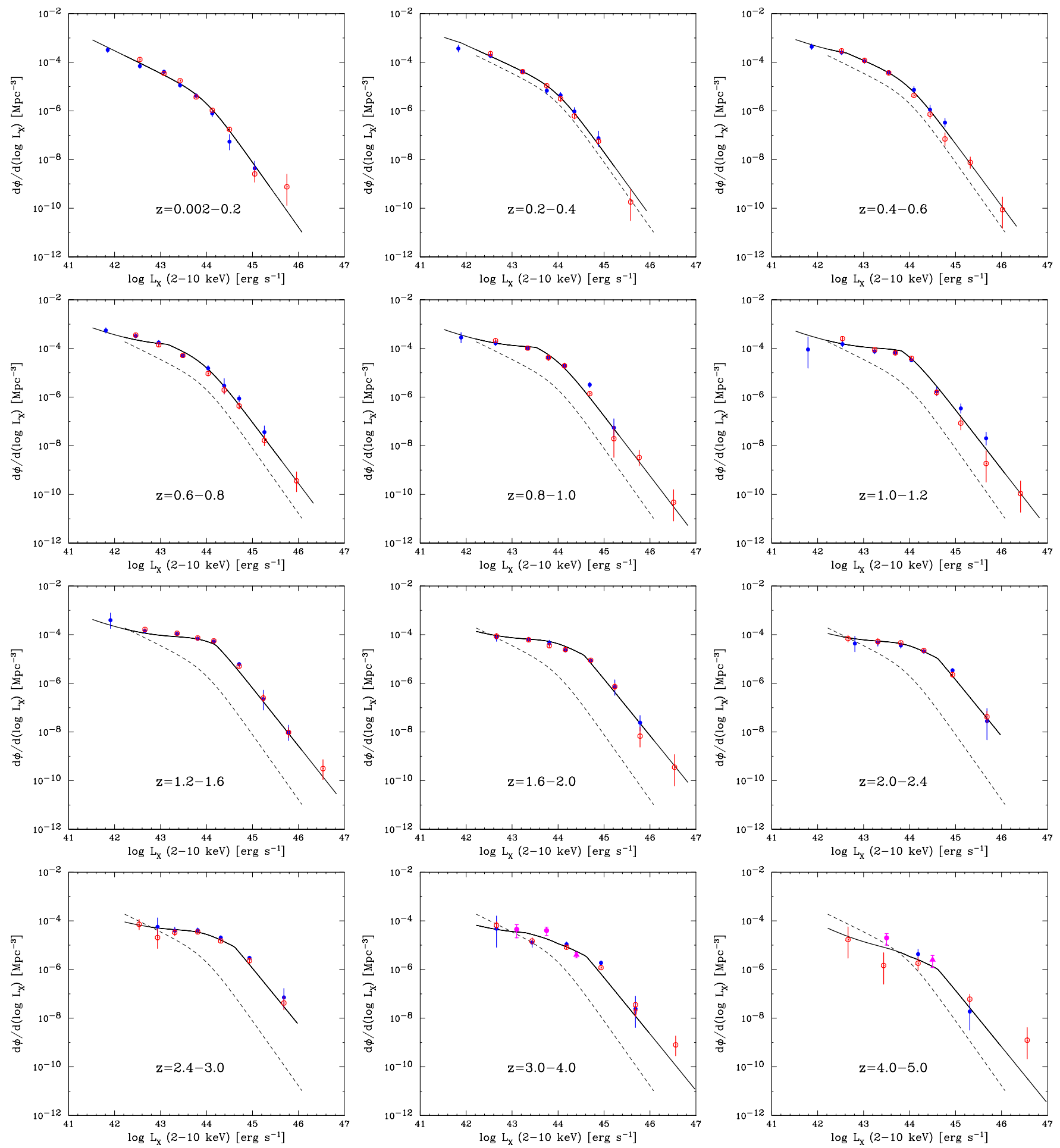

Figure 10. De-absorbed, rest frame 2-10 keV XLF of AGNs at different redshift ranges (CTN AGNs only). The solid curve represents the best-fit XLF at the central redshift in each $z$ bin. The dashed curve is that in the local universe. Blue (red) data points are plotted according to the " $N^{\text {obs }} / N^{\text {mdl }}$ method" with $1 \sigma$ Poisson errors by using the hard (soft)-band sample. The magenta points in the $z=3.0-4.0$ and $z=4.0-5.0$ panels are taken from Fiore et al. (2012).

(A color version of this figure is available in the online journal.)

$\alpha 2=-0.1$ indicates an "upsizing" evolution instead (i.e., the number ratio of less luminous AGNs to more luminous AGNs is larger at earlier epochs). This is what is expected from the hierarchical structure formation in the early universe. Thus, the SMBH growth must be correctly described by "up-downsizing". To firmly establish this behavior, it is critical to determine the space number density of all AGNs at $z \gtrsim 3$ in both the lowest and highest luminosity ranges with better accuracies.

\subsection{LADE Model}

As noted in Figures 10 and 11, the shape of the XLF at $z=1-3$ is quite different from that in the local universe in the sense that the slope at the low-luminosity range is significantly flatter than those observed at lower redshifts. This trend can be well reproduced by the LDDE model. Here, we check if the LADE model of the XLF proposed by Aird et al. (2010) 


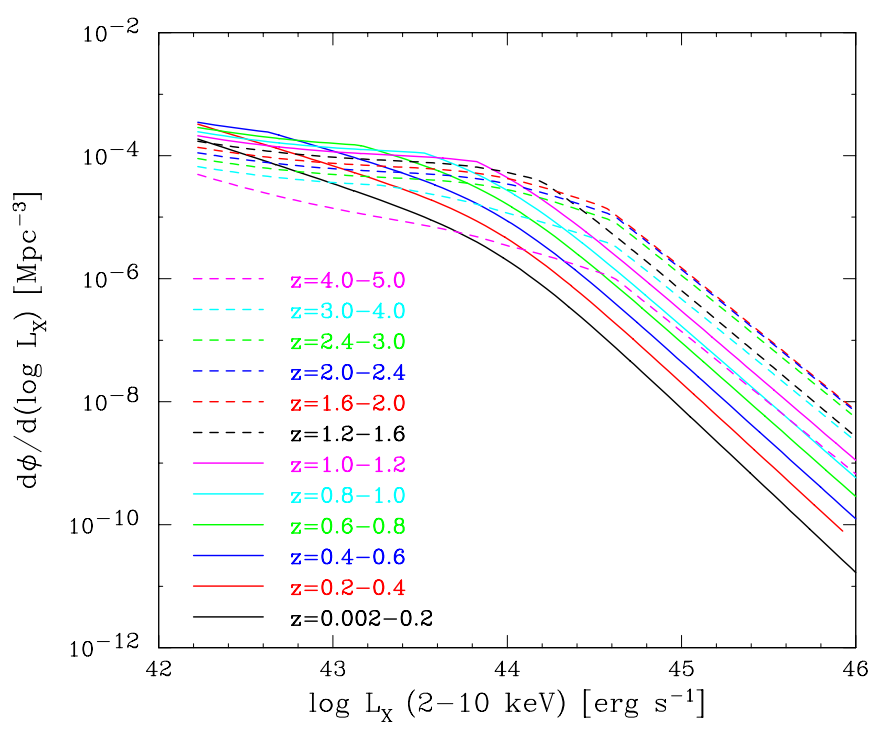

Figure 11. Comparison of the best-fit XLF shape between different redshifts (CTN AGNs only).

(A color version of this figure is available in the online journal.)

also gives a good description of our data. Unlike the LDDE, the LADE assumes a constant relative shape of the XLF in the logarithmic scales over the full redshift range, and its break luminosity and normalization is given as a function of redshift. We perform an ML fit to the whole sample by adopting the same formulation of the XLF as given in Aird et al. (2010). A chi-squared test for the two-dimensional histograms of flux and redshift between the best-fit model and data yields $\chi^{2}=207.1$ $($ dof $=114)$. The LADE model is thus rejected with a $p$ value of $<10^{-7}$. We infer that it is difficult to distinguish the LDDE and LADE models in Aird et al. (2010) because of the smaller number of samples used there; indeed Aird et al. (2010) show that the LDDE gives a better fit to their data than the LADE, although the difference is not significant.

\subsection{Comparison with Previous Works}

The parameters of the AGN XLF are better constrained than in any of previous works thanks to our large sample size $(\approx 15$ and $\approx 4$ times larger than those used by U03 and H05, respectively). Here, we compare them with those of the LDDE model by $\mathrm{U} 03$ and by $\mathrm{H} 05$ as representative ones. Although the direct comparison with $\mathrm{U} 03$ is not trivial as the formulation of the XLF in U03 is simpler than ours (e.g., $\beta_{1}=0$ is assumed in U03), the overall parameters are in good agreement between our work and U03 except for $\gamma_{2}$. The overall shape of our XLF derived for all CTN AGNs is almost consistent with that by H05 derived only for type-1 AGNs (see their Table 5) within the errors except for $\alpha$ (= $=\alpha 1$ in our paper), which is found to be slightly larger $(\alpha 1=0.29 \pm 0.02)$ than in $\mathrm{H} 05(\alpha=0.21 \pm 0.04)$. Note that the $z_{\mathrm{c}, 44}=0.21 \pm 0.04$ parameter defined in $\mathrm{H} 05$ can be converted to $z_{\mathrm{c}}=1.96 \pm 0.15$ with $\alpha=0.21(=\alpha 1$ in our paper), and thus agrees with our result $\left(z_{\mathrm{c}}=1.86 \pm 0.07\right)$. Our best-fit model has steeper slopes in the double power-law form for the local XLF, $\gamma_{1}=0.96 \pm 0.04$ and $\gamma_{2}=2.71 \pm 0.09$, than those obtained by $\mathrm{H} 05$. This can be explained by the luminosity dependence of the absorbed-AGN fraction. Our local XLF is well consistent with the Ballantyne (2014) result as determined by the "multiband" fit.

We also determine the evolution of the absorption fraction with an unprecedented accuracy, $a 1=0.48 \pm 0.05$, in the form

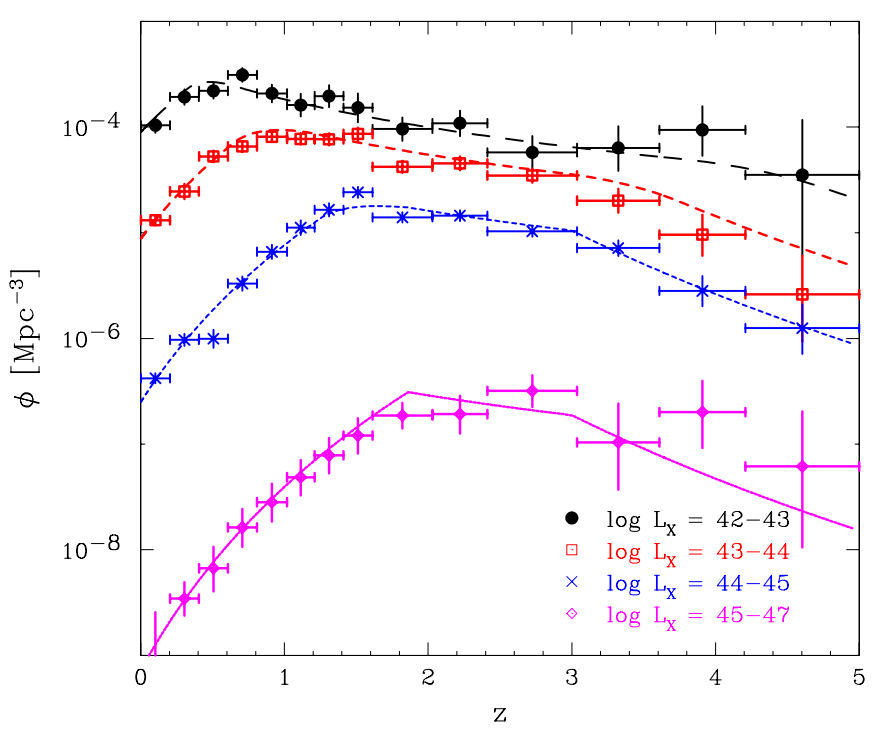

Figure 12. Comoving number density of AGNs plotted against redshift in different luminosity bins (CTN AGNs only). The curves are the best-fit model, and the data points are calculated from either the soft- or hard-band sample (see Section 6).

(A color version of this figure is available in the online journal.)

of $(1+z)^{a 1}$ that is saturated above $z=2$. La Franca et al. (2005) model the redshift evolution of the absorption fraction by a different parameterization, adopting a linear function of $z$ for the fraction of AGNs with $\log N_{\mathrm{H}}<21$. According to their best-fit model (model 4), where the constant $N_{\mathrm{H}}$ distribution is assumed over $\log N_{\mathrm{H}}=21-25$, the fraction of absorbed CTN AGNs $\left(\log N_{\mathrm{H}}=22-24\right)$ in the total CTN AGNs $\left(\log N_{\mathrm{H}}<24\right)$ at $\log L_{X}=44$ is 2.3 times higher at $z=2$ than at $z=0$. This corresponds to $a 1 \approx 0.75$ when modeled by $(1+z)^{a 1}$. Similarly, Hasinger (2008) obtain $(1+z)^{0.62 \pm 0.11}$ that is saturated at $z>2$. The reason why both La Franca et al. (2005) and Hasinger (2008) obtain larger indices than ours could be the difference in the adopted absorption fraction in the local universe. Both of them utilize the HEAOI samples, from which somewhat smaller absorption fractions are estimated compared with the Swift/ BAT and MAXI results. In the La Franca et al. (2005) model, the fraction of CTN AGNs in the total CTN AGNs is $\approx 0.25$ at $\log L_{X}=44$, which can be converted to $\psi_{43.75}^{0} \approx 0.31$ with $\beta=0.24$. This value is similar to that presented in Hasinger (2008), while it is smaller than our result obtained from the Swift/BAT sample, $\psi_{43.75}^{0}=0.43 \pm 0.03$. The reason for the discrepancy is unclear but may be attributed to the statistical error due to the small size of the HEAO1 A2 sample (Piccinotti et al. 1982) and/or incompleteness of the HEAO1 A1 and A3 sample (Grossan 1992). Note that our best-fit slope is larger than that in the model by Ballantyne et al. (2006), $a 1 \approx 0.3$, where the absorption fraction is assumed to be saturated above $z=1.0$. Treister \& Urry (2006) obtain a similar slope to ours, $a 1 \approx 0.4 \pm 0.1$ without saturation up to $z=4$, by correcting for selection biases due to the low completeness $(53 \%)$ in their sample.

\section{STANDARD POPULATION SYNTHESIS MODEL OF THE XRB}

\subsection{Model Predictions}

We have constructed a new XLF of AGNs by utilizing one of the largest samples with a high degree of identification completeness combined from surveys in different energy bands. We 


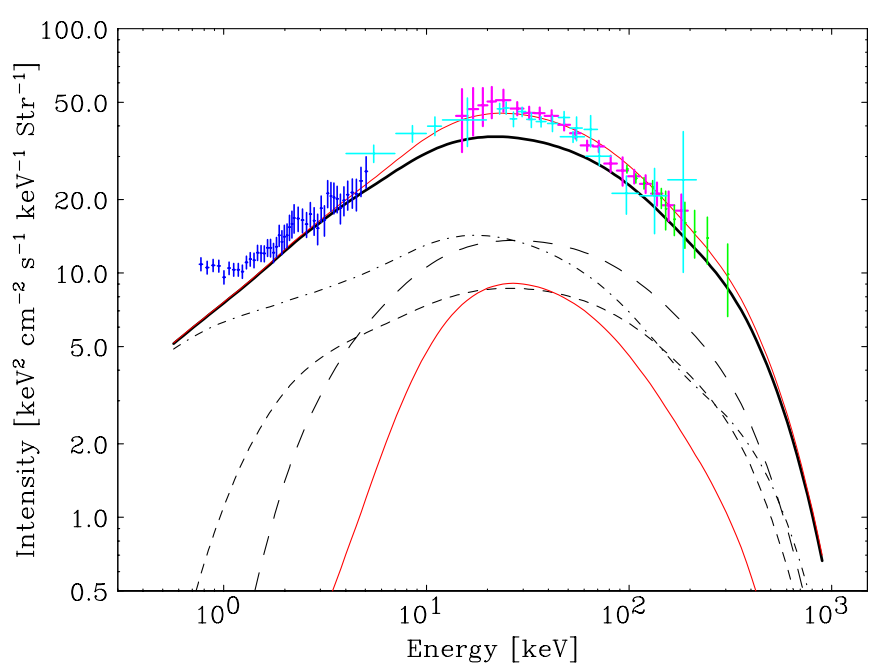

Figure 13. XRB spectrum calculated from our AGN population synthesis model (upper solid curve, red) compared with the observed data by various missions (Ajello et al. 2008). Middle solid curve (black): the integrated spectrum of CTN AGNs $\left(\log N_{\mathrm{H}}<24\right)$. Lower solid curve (red): that of CTK AGNs $\left(\log N_{\mathrm{H}}=\right.$ 24-26). Long-dashed curve (black): that of AGNs with $\log N_{\mathrm{H}}=23-24$. Shortdashed curve (black): that of AGNs with $\log N_{\mathrm{H}}=22-23$. Dot-dashed curve (black): that of AGNs with $\log N_{\mathrm{H}}<22$. Data points in the $0.8-5 \mathrm{keV}$ (blue), 4-215 keV (cyan), 14-195 keV (magenta), and 100-300 keV (green) bands refer to the XRB spectra observed with ASCA/SIS (Gendreau et al. 1995), INTEGRAL (Churazov et al. 2007), Swift/BAT (Ajello et al. 2008), and HEAO1 A4 (Gruber et al. 1999), respectively.

(A color version of this figure is available in the online journal.)

also model the absorption and photon index functions on the basis of a hard X-ray ( $>15 \mathrm{keV}$ ) selected AGN sample in the local universe, for which detailed spectral information is available. The redshift dependence of the absorption function is taken into account, whose evolution index $a 1$ is simultaneously determined along with the XLF parameters. We consider the contribution of CTK AGNs by assuming that their number density at a given luminosity and redshift is the same as that of obscured CTN AGNs. The combination of the XLF, absorption function, and photon index function with the template broadband spectra of AGNs enables us to establish a new population synthesis model of the XRB. In this section, we examine the basic properties of the model.

Figure 13 shows the integrated broadband spectrum of the whole AGNs at $z=0.002-5.0$, with $\log L_{X}=41-47$ predicted from our model. The spectrum of each AGN is modeled by the "template spectrum" presented in Section 4, which is given as a function of luminosity, column density, and photon index. The data points represent the measurements of the XRB observed with various missions, including HEAOI A4 in the $100-300 \mathrm{keV}$ band (Gruber et al. 1999), Swift/BAT in the 14-195 keV band (Ajello et al. 2008), and INTEGRAL in the 4-215 keV band (Churazov et al. 2007). A good agreement is confirmed between the model prediction and the hard XRB, supporting the overall validity of our model, including the fraction of CTK AGNs and the reflection strengths from the accretion disk and torus based on the luminosity- and redshift- dependent unified scheme (Section 4). Effects by changing these model parameters will be examined in Section 7.2.

There are discrepancies in the absolute flux measurements of the XRB between different missions, most probably due to calibration uncertainties. These issues are discussed in detail by, e.g., Barcons et al. (2000) for the XRB below $10 \mathrm{keV}$ and by
Ajello et al. (2008) above $10 \mathrm{keV}$. In Figure 13, for clarity, we only plot the ASCA result obtained by Gendreau et al. (1995) as the representative data of the XRB in the $0.8-5 \mathrm{keV}$ band. The XRB spectrum obtained by the HEAO1 A2 experiment gives systematically smaller fluxes in the energy range below $10 \mathrm{keV}$ than most of more recent missions. The maximum flux is reported by De Luca \& Molendi (2004) with XMM-Newton, which is $40 \%$ higher than that of the original HEAO1 A2 result (Marshall et al. 1980). The reasons are yet unclear. In addition, we do not include the emission from populations other than AGNs in our model. For instance, clusters of galaxies could contribute to the XRB by $\sim 10 \%$ level at $1 \mathrm{keV}$. For these reasons, we mainly discuss our population synthesis model on the basis of the hard XRB above $10 \mathrm{keV}$, where the contribution from AGNs is dominant.

The contributions from all (CTN+CTK) AGNs per unit $z$ per unit $\log L_{\mathrm{X}}$ to the XRB flux in the $2-10 \mathrm{keV}$ and $10-40 \mathrm{keV}$ bands are shown by the contours in Figure 14 (left) and (right), respectively. As noted from the figures, AGNs with $\log L_{X} \approx 43.8$ $(\approx 43.7)$ at $z \approx 1.1(\approx 1.0)$ make the largest contribution to the $\mathrm{XRB}$ in the $2-10 \mathrm{keV}(10-40 \mathrm{keV})$ band. Figure 15 (left) and (right) plot the differential XRB intensity per unit $\log L_{X}$ in a redshift region of $z=0.002-5$, and per unit $z$ in a luminosity region of $\log L_{X}=41-47$, respectively.

The predicted $\log N-\log S$ relation of AGNs in the $0.5-2 \mathrm{keV}$, 2-10 keV, 8-24 keV, and 10-40 keV bands are plotted in Figure 16. We separately plot the contributions from AGNs at different redshift ranges $(z<1, z=1-2, z=2-3$, and $z=3-5)$ and from those with different absorptions $\left(\log N_{\mathrm{H}}=20-22\right.$, 22-24, 24-26). Figure 17 shows the fractions of CTK AGNs $\left(\log N_{\mathrm{H}}=24-26\right)$ and obscured AGNs $\left(\log N_{\mathrm{H}}=22-26\right)$ in the total AGNs $\left(\log N_{\mathrm{H}} \leqslant 26\right)$ as a function of flux predicted from surveys in the $2-10 \mathrm{keV}$ (left) and $10-40 \mathrm{keV}$ (right) bands. The CTK AGN fraction reaches $\approx 20 \%$ at $S \sim 10^{-16} \mathrm{erg} \mathrm{cm}^{-2} \mathrm{~s}^{-1}$ in the 2-10 keV band, the flux limit of Chandra deep surveys. We find that the observed CTK AGN fractions at various flux limits in the $2-10 \mathrm{keV}$ (or $0.5-8 \mathrm{keV}$ ) band reported by Tozzi et al. (2006), Hasinger et al. (2007), Brunner et al. (2008), and Brightman \& Ueda (2012) are generally in good agreement with the model prediction. In the 10-40 keV band, our model is consistent with the observed CTK fraction at $S \sim 10^{-11} \mathrm{erg} \mathrm{cm}^{-2} \mathrm{~s}^{-1}$ observed by the Swift/BAT 9 month survey performed in the 14-195 keV band (Tueller et al. 2008; Ichikawa et al. 2012) and with the upper limit $(<0.23$ at a $90 \%$ confidence level) obtained from the first $N u S T A R$ extragalactic survey in the 8-24 keV band (Alexander et al. 2013). In our baseline model, the intrinsic fraction of CTK AGNs among the whole AGNs at $\log L_{\mathrm{X}}=43.75$ is $30 \pm 2 \%$ at $z=0$, $37 \pm 2 \%$ at $z=1$, and $42 \pm 2 \%$ at $z \geqslant 2$, which are calculated as $f_{\text {CTK }} \psi_{43.75}(z) /\left[1+f_{\text {CTK }} \psi_{43.75}(z)\right]$. They are fully consistent with the results obtained by Brightman \& Ueda (2012) from the CDFS data at $z>0.1$. Note that using the $S$ wift/BAT 3 yr survey, Burlon et al. (2011) report a slightly smaller CTK fraction of $20_{-6}^{+9} \%$ than the above value, though within the errors, because they do not include heavily CTK AGNs with $\log N_{\mathrm{H}}>25$.

Figure 18 plots the comoving number density of CTK AGNs with different lower luminosity limits as a function of redshift predicted from the baseline model. For comparison, the estimates from X-ray stacking analyses obtained by Fiore et al. $(2008,2009)$ are over-plotted. The result by Fiore et al. (2008) at $z=1.2-2.6$ for $\log L_{X}>43$ (open circle) agrees well with our model. More recent results reported by Fiore et al. (2009) from the COSMOS data (filled squares) at $z=0.7-1.2$ 

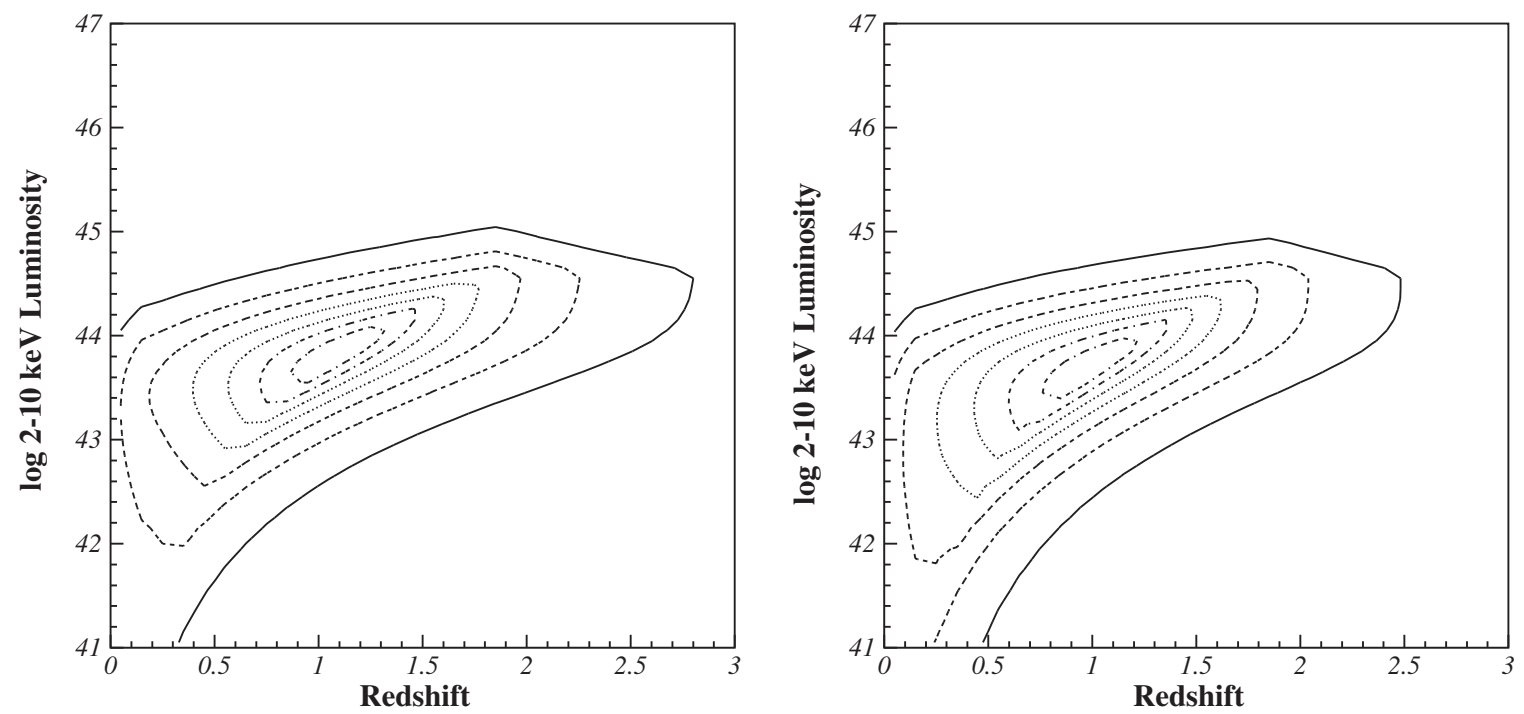

Figure 14. Left: contour plot showing the contribution of all (CTN+CTK) AGNs per unit $z$ and $\log L_{X}$ to the $2-10 \mathrm{keV}$ XRB. The intervals are constant in linear scale. Right: the same but for the $10-40 \mathrm{keV}$ XRB.
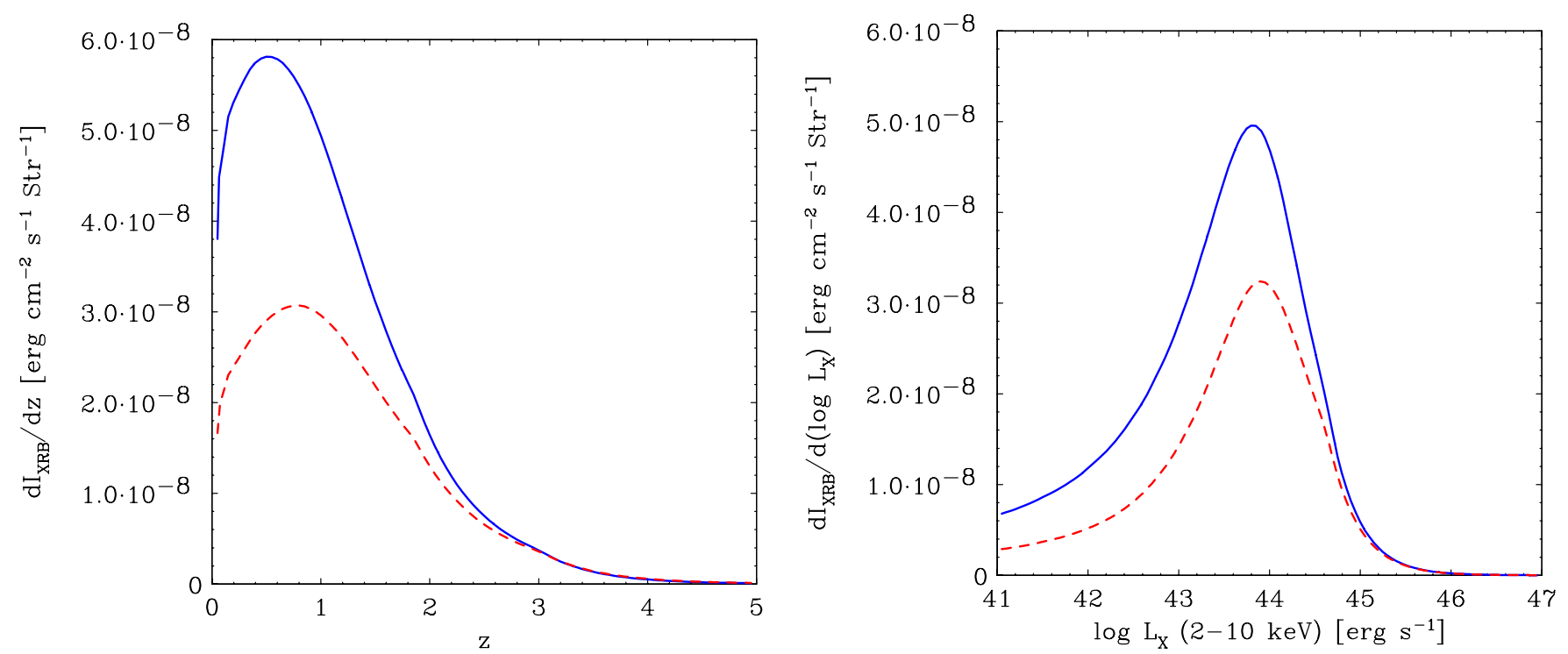

Figure 15. Left: Differential contribution to the $2-10 \mathrm{keV}$ (red dashed) or $10-40 \mathrm{keV}$ (blue solid) XRB intensity as a function of redshift (in units of per $z$ ) from all (CTN+CTK) AGNs with $\log L_{\mathrm{X}}=41-47$. Right: that as a function of luminosity (in units of per $\log L_{\mathrm{X}}$ ) from all AGNs at $z=0.002-5$.

(A color version of this figure is available in the online journal.)

$\left(\log L_{\mathrm{X}}>43.5\right)$ and at $z=1.2-2.2\left(\log L_{\mathrm{X}}>44\right)$ are within a factor of $\sim 2$ from our baseline model, which would be acceptable by considering possible uncertainties in the luminosity range of the samples. In the figure, we also plot the estimate based on X-ray detected heavily obscured AGNs in the CDFS at $z=1.4-2.6$ with $\log L_{\mathrm{X}} \gtrsim 43$ obtained by Alexander et al. (2011) (filled circle), who updated the Daddi et al. (2007) result using deeper $\mathrm{X}$-ray data and new analyses. Our prediction is by a factor of $\sim 3$ higher than their result, which should be regarded as a conservative lower limit (Alexander et al. 2011).

\subsection{Constraints on Compton-thick AGN Fraction}

As described above, our population synthesis model has the following parameters that are fixed in the main analysis of Section 6: (1) the fraction of CTK AGNs $f_{\text {СTK }}=1.0$, (2) the strength of the reflection component from the accretion disk $R_{\text {disk }}=0.5$, and (3) a mean photon index and its scatter of
$\overline{\Gamma_{1}}=1.94$ and $\Delta \Gamma_{1}=0.09$ for type- 1 AGNs, and $\overline{\Gamma_{2}}=1.84$ and $\Delta \Gamma_{2}=0.15$ for type-2 AGNs. In this subsection, we evaluate the dependence of model predictions on these fixed parameters and discuss constraints on the fraction of CTK AGNs. As the boundary condition that must be reproduced from the XRB model, we use the XRB intensity integrated in the 20-50 keV band, $I_{\mathrm{XRB}, 20-50}$. Considering the systematic uncertainties between different missions (see Table 2 of Ajello et al. 2008), we conservatively adopt $I_{\mathrm{XRB}, 20-50}=(5.7-6.7) \times$ $10^{-8} \mathrm{erg} \mathrm{cm}^{-2} \mathrm{~s}^{-1} \mathrm{Str}^{-1}$ as the constraint; the minimum and maximum values are obtained by BeppoSAX (Fronetera et al. 2007) and INTEGRAL (Churazov et al. 2007), respectively, when we adopt Gruber et al. (1999) as the HEAOI's result. We also check the AGN source counts in the $2-8 \mathrm{keV}$ band at a representative flux of $S=2.7 \times 10^{-16} \mathrm{erg} \mathrm{cm}^{-2} \mathrm{~s}^{-1}$, which sensitively depends on the assumed $f_{\text {Стк }}$ parameter, to be compared with the Chandra result obtained by Lehmer et al. (2012), $N\left(>S=2.7 \times 10^{-16}\right)=4290 \pm 240 \mathrm{deg}^{-2}$ 

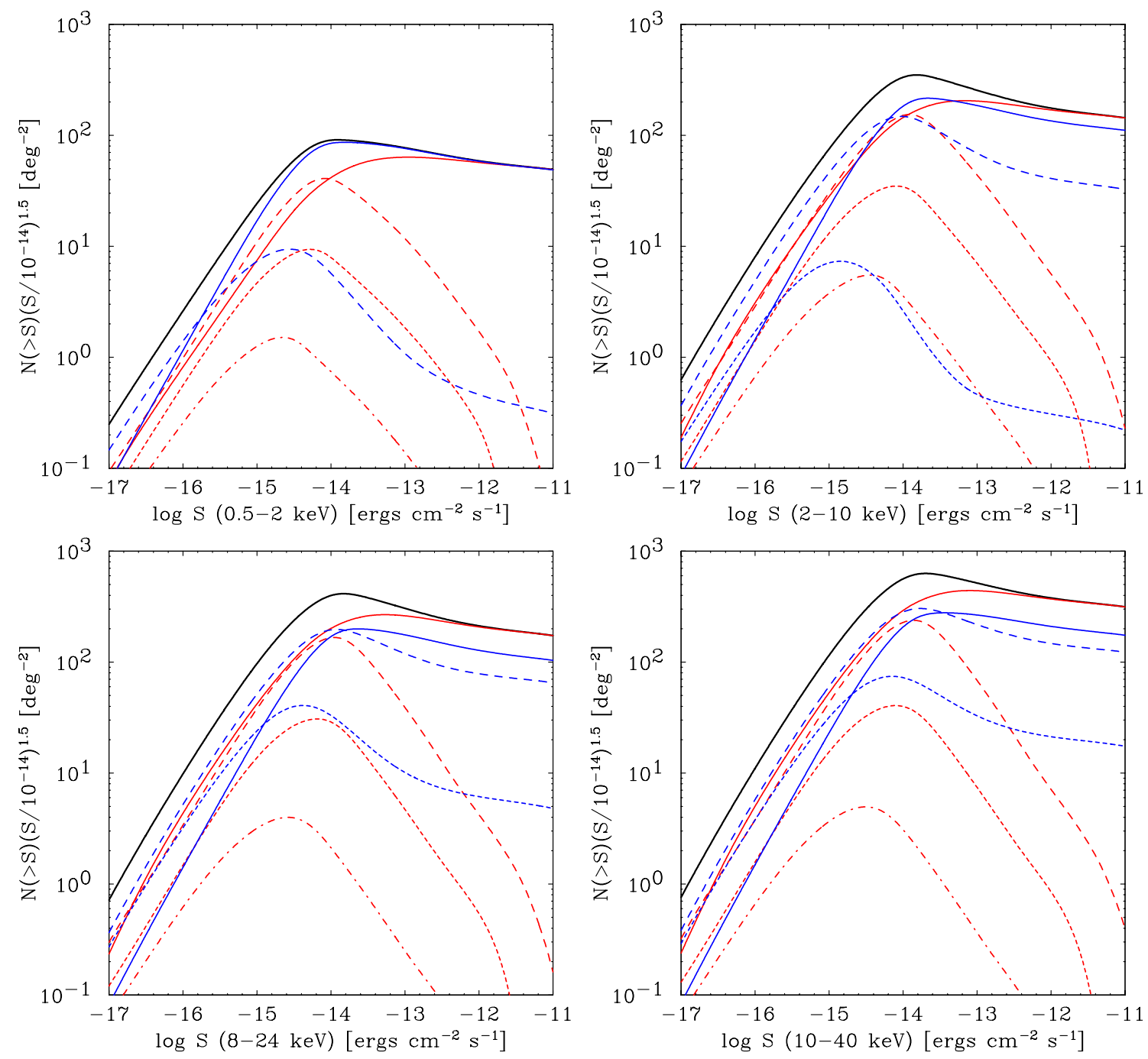

Figure 16. Upper left: predicted integrated $\log N-\log S$ relations in the $0.5-2 \mathrm{keV}$ band from our baseline model normalized as $N(>S)\left(S / 10^{-14}\right)^{1.5}$. The red curves correspond to those in different redshifts (solid: $z<1$; long-dashed: $z=1-2$; short-dashed: $z=2-3$; dot-dashed: $z=3-5$ ), and blue curves to those with different absorptions (solid: $\log N_{\mathrm{H}}=20-22$; long-dashed: $\log N_{\mathrm{H}}=22-24$; short-dashed: $\log N_{\mathrm{H}}=24-26$ ). Top right: the same but for the 2-10 keV band. Lower left: the same but for the $8-24 \mathrm{keV}$ band. Lower right: the same but for the $10-40 \mathrm{keV}$ band. Predicted $\log N-\log S$ relations are available in numerical form at http://www.kusastro.kyoto-u.ac.jp/ yueda/xrb2014.html

(A color version of this figure is available in the online journal.)

(for AGNs only). At fainter fluxes, the contribution of normal galaxies becomes more important, which are difficult to be unambiguously distinguished from AGNs (Xue et al. 2011).

Since these fixed parameters affect the fitting results of the XLF and absorption function, we repeat ML fit to the list of our AGN sample by replacing the default parameters with different values. For simplicity, only one set of parameters (i.e., either $f_{\mathrm{CTK}}, R_{\mathrm{disk}}, \overline{\Gamma_{1,2}}$, or $\left.\Delta \Gamma_{1,2}\right)$ is changed from the default values. This enables us to estimate the error for a single parameter by ignoring the coupling between them. Table 5 summarizes the results obtained for different values of the fixed parameters. Since we find the XLF parameters are not significantly different over the statistical errors, we only show the evolution index in the absorption function, $a 1$. The predicted XRB intensity in the $20-50 \mathrm{keV}$ band and the $2-8 \mathrm{keV}$ source count at $S=2.7 \times 10^{-16} \mathrm{erg} \mathrm{cm}^{-2} \mathrm{~s}^{-1}$ are listed. Figure 19 compares the integrated spectra for the cases of $f_{\text {CTK }}=0.5$ and $f_{\text {CTK }}=2.0$ (short-dashed, red) and $R_{\text {disk }}=0.25$ and $R_{\text {disk }}=1.0$ (long-dashed, blue) with our baseline model (black). Taking the $20-50 \mathrm{keV}$ XRB intensity $I_{\mathrm{XRB}, 20-50}=$
(5.7-6.7) $\times 10^{-8} \mathrm{erg} \mathrm{cm}^{-2} \mathrm{~s}^{-1} \mathrm{Str}^{-1}$ as the observational constraint, we constrain that $f_{\text {CTK }}=0.5-1.6$ in the case of $R_{\text {disk }}=$ 0.5 ; for this range of $f_{\text {CTK }}$, we confirm that the predicted source count at $S=2.7 \times 10^{-16}$ in the $2-8 \mathrm{keV}$ band is consistent with the observed one, $N\left(>S=2.7 \times 10^{-16}\right)=4290 \pm 240 \mathrm{deg}^{-2}$. As discussed in many previous works, there are degeneracies between the estimate of CTK AGN fraction and the strength of Compton reflection components in order to reproduce the XRB spectrum. From results listed in Table 5, we can roughly estimate that the best-estimate of $f_{\text {CTK }}$ will be changed by $+50 \%$ and $-50 \%$ when we assume $R_{\text {disk }}=0.25$ and 1.0 , respectively, although the choice of $R_{\text {disk }}=0.5$ in our baseline model is the most reasonable from observations of local AGNs (Section 4).

\subsection{Comparison with Previous XRB Models}

We compare our new population synthesis model of the XRB with major previous works published after 2003: U03, Ballantyne et al. (2006), Gilli et al. (2007), Treister et al. (2009), and Akylas et al. (2012). All these models, including ours, assume that the CTK AGNs follow the same cosmological 

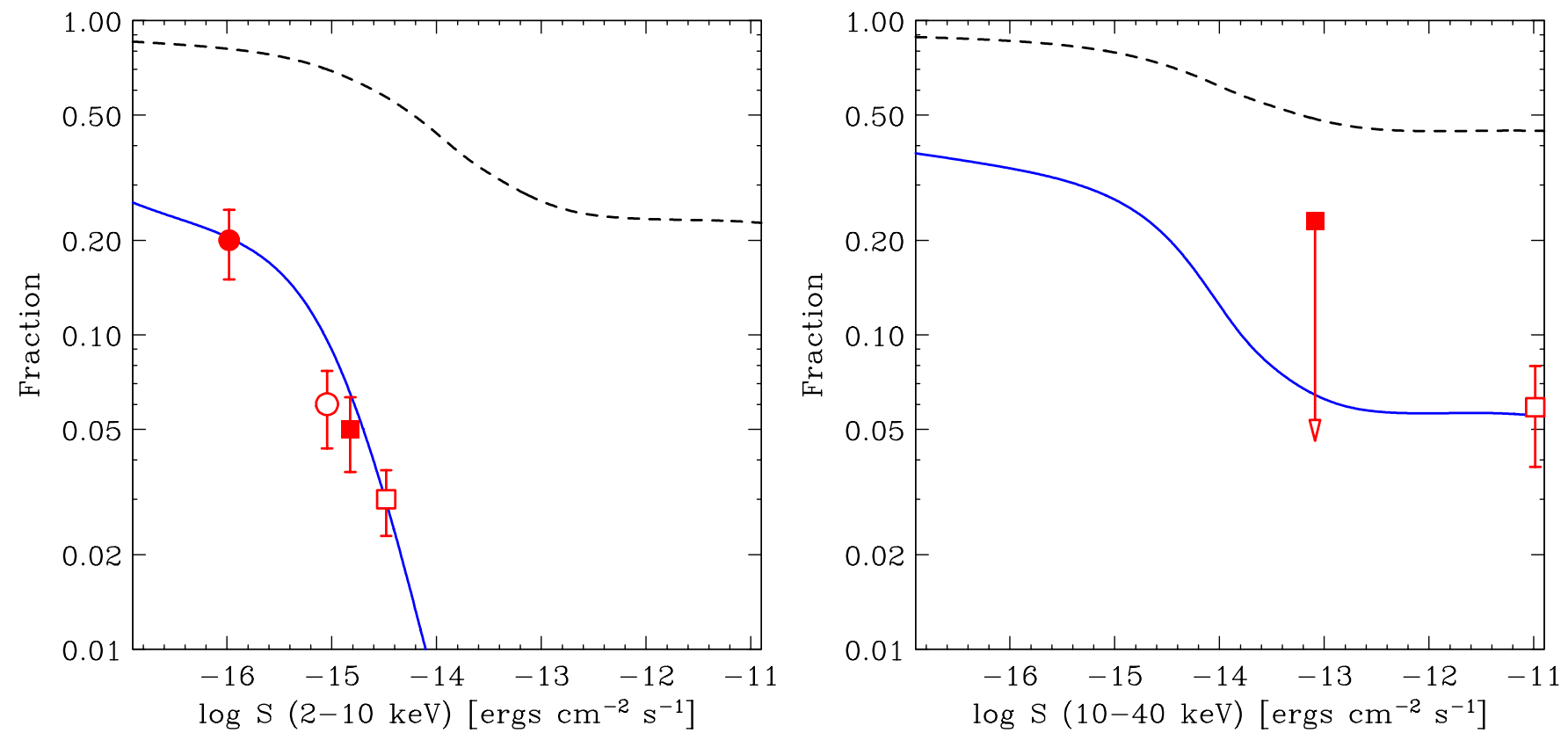

Figure 17. Left: fractions of CTK AGNs ( $\log N_{\mathrm{H}}=24-26$, solid blue) and obscured AGNs ( $\log N_{\mathrm{H}}=22-26$, dashed black) in the total AGNs given as a function of flux in the 2-10 keV band, predicted from our baseline model. The data points correspond to the observed CTK AGN fractions by Brightman \& Ueda (2012; filled circle), Brunner et al. (2008; open circle), Tozzi et al. (2006; filled square), and Hasinger et al. (2007; open square), from left to right. Here, the result by Brightman \& Ueda (2012) is plotted by converting the $0.5-8 \mathrm{keV}$ flux to the $2-10 \mathrm{keV}$ one by assuming a photon index of 1.4 . Right: the same but for the $10-40 \mathrm{keV}$ flux. The arrow denotes the $90 \%$ confidence upper limit of the CTK fraction obtained by NuSTAR in the 8-24 keV band (Alexander et al. 2013), and the right data point is that from the Swift/BAT 9 month survey in the 14-195 keV band (Tueller et al. 2008; Ichikawa et al. 2012). The fluxes are converted into the $10-40 \mathrm{keV}$ band by assuming a photon index of 1.8 .

(A color version of this figure is available in the online journal.)

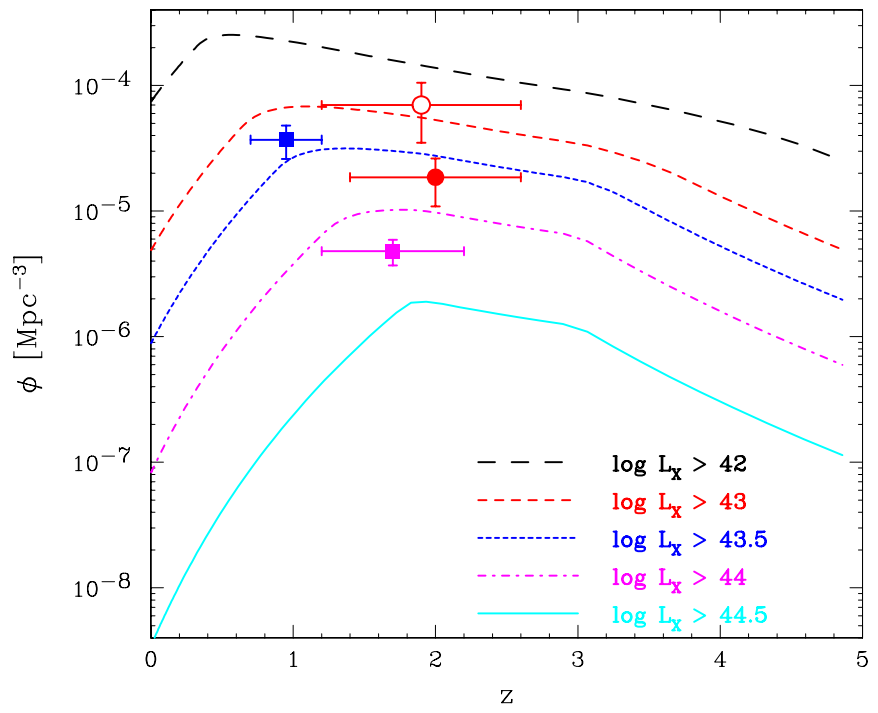

Figure 18. Comoving number density of CTK AGNs with different lower limits for the X-ray luminosity predicted from our baseline model. The data points represent the estimates by Alexander et al. (2011) for $\log L_{X} \gtrsim 43$ (filled circle, red), Fiore et al. (2008) for $\log L_{X}>43$ (open circle, red), and Fiore et al. (2009) for $\log L_{X}>43.5$ (filled square, blue) and for $\log L_{X}>44$ (filled square, magenta).

(A color version of this figure is available in the online journal.)

evolution of CTN AGNs and introduce the $f_{\text {CTK }}$ (or its equivalent) parameter. Table 6 summarizes the details of the ingredients in each model: the XLF, the evolution index of the absorption fraction $a 1$ ( $a 1=0$ if no evolution), $f_{\text {СтK }}$ with the range of column density of CTK AGNs, spectral parameters $(\bar{\Gamma}$ and $\Delta \Gamma)$, reflection strength, high energy cutoff), and the predicted XRB

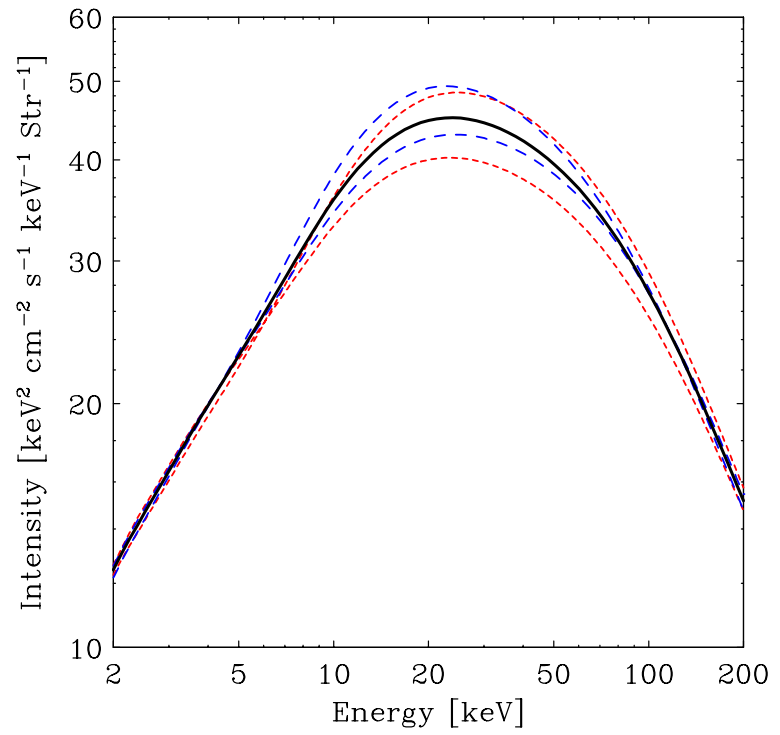

Figure 19. The predicted XRB spectra by assuming different fractions of CTK AGNs relative to obscured CTN AGNs (short-dashed, red; $f_{\text {CTK }}=2$ and 0.5 from upper to lower), or different reflection strengths from the accretion disk (long-dashed, blue; $R_{\text {disk }}=1.0$ and 0.25 from upper to lower). The baseline model $\left(f_{\text {CTK }}=1.0\right.$ and $R_{\text {disk }}=0.5$ ) is plotted by the solid line (solid, black).

(A color version of this figure is available in the online journal.)

intensity at $25 \mathrm{keV}$. Akylas et al. (2012) intensively explore the degeneracies between these parameters by fitting the XRB spectra with the model predictions. In Table 6, only a representative set of the parameters that fits the XRB data is listed (taken from their Figure 1).

All these works except Gilli et al. (2007) utilize the $2-10 \mathrm{keV}$ XLF of the whole CTN AGNs obtained by U03. Gilli et al. 
Table 5

Comparison of Model Predictions

\begin{tabular}{lccc}
\hline \hline Changed Parameters & $a 1^{\mathrm{a}}$ & $\begin{array}{c}I_{\mathrm{XRB}, 20-50}^{\mathrm{b}} \\
\left(10^{-8} \mathrm{erg} \mathrm{cm}^{-2} \mathrm{~s}^{-1} \mathrm{Str}^{-1}\right)\end{array}$ & $\begin{array}{c}N(>S)^{\mathrm{c}} \\
\left(\mathrm{deg}^{-2}\right)\end{array}$ \\
\hline Baseline model & $0.48 \pm 0.05$ & 6.39 & 4300 \\
$f_{\text {CTK }}=2.0$ & $0.34 \pm 0.08$ & 6.87 & 4500 \\
$f_{\text {CTK }}=0.5$ & $0.55 \pm 0.06$ & 5.72 & 4120 \\
$R_{\text {disk }}=1.0$ & $0.39 \pm 0.07$ & 6.91 & 4280 \\
$R_{\text {disk }}=0.25$ & $0.54 \pm 0.06$ & 6.13 & 4320 \\
$\bar{\Gamma}_{1}=1.97$ and $\overline{\Gamma_{2}}=1.88$ & $0.51 \pm 0.06$ & 5.88 & 4270 \\
$\Gamma_{1}=1.91$ and $\overline{\Gamma_{2}}=1.80$ & $0.44 \pm 0.07$ & 6.91 & 4330 \\
$\Delta \Gamma_{1}=0.14$ and $\Delta \Gamma_{2}=0.21$ & $0.48 \pm 0.05$ & 6.82 & 4340 \\
$\Delta \Gamma_{1}=0.04$ and $\Delta \Gamma_{2}=0.10$ & $0.46 \pm 0.06$ & 6.09 & 4230 \\
\hline
\end{tabular}

Notes. The other parameters are the same as in the baseline model.

a The $a 1$ parameter in the absorption function.

b Predicted XRB intensity in the $20-50 \mathrm{keV}$ band.

c Predicted AGN counts at $S=2.7 \times 10^{-16} \mathrm{erg} \mathrm{cm}^{-2} \mathrm{~s}^{-1}$ in the $2-8 \mathrm{keV}$ band. The observed value in the CDFS is $4290 \pm 240 \mathrm{deg}^{-2}$ (Lehmer et al. 2012).

Table 6

Summary of Population Synthesis Models

\begin{tabular}{lllllcccc}
\hline \hline References & XLF & $a 1^{\mathrm{a}}$ & $\bar{\Gamma}$ & $\Delta \Gamma$ & $\begin{array}{c}E_{\mathrm{c}} \\
(\mathrm{keV})\end{array}$ & $f_{\mathrm{CTK}}\left(\log N_{\mathrm{H}}\right)^{\mathrm{b}}$ & $R_{\mathrm{total}} \mathrm{c}$ & $\begin{array}{c}E I_{\mathrm{XRB}}(E) \mathrm{at} 30 \mathrm{keV} \\
\left(\mathrm{keV} \mathrm{cm}^{-2} \mathrm{~s}^{-1} \mathrm{Str}^{-1}\right)\end{array}$ \\
\hline$(1)$ & $\mathrm{U} 03$ & 0 & 1.9 & 0 & 500 & $0.63(24-25)$ & 1.0 & 50 \\
$(2)^{\mathrm{d}}$ & $\mathrm{U} 03$ & 0.3 & 1.9 & 0 & 375 & $0.5(24-25)$ & 1.0 & 52 \\
$(3)$ & $\mathrm{H} 05$ & 0 & 1.9 & 0.2 & 200 & $1.05(24-26)$ & $\approx 1.0$ & 40 \\
$(4)$ & $\mathrm{U} 03$ & 0.4 & 1.9 & 0 & 300 & $0.17(24-26)$ & 1.2 & 42 \\
$(5)^{\mathrm{e}}$ & $\mathrm{U} 03$ & 0 & 1.88 & 0.15 & 230 & $0.1(24-25)$ & $\approx 1.0$ & 44 \\
This work & This work & 0.48 & $1.94,1.88^{\mathrm{f}}$ & $0.09,0.15^{\mathrm{f}}$ & 300 & $1.0(24-26)$ & $0.5+R_{\text {torus }}$ & 45 \\
\hline
\end{tabular}

Notes.

a The evolution index of the absorption fraction modeled as $\propto(1+z)^{a 1}$.

b The number ratio of CTK AGNs to obscured CTN AGNs with $\log N_{\mathrm{H}}=22-24$. In the parentheses, the region of column densities (log $N_{\mathrm{H}}$ ) considered for CTK AGNs is given.

c The relative strength of the total reflection components as modeled by the pexrav code (Magdziarz $\&$ Zdziarski 1995$), R_{\text {tot }} \equiv \Omega / 2 \pi$, where $\Omega$ is the solid angle of the reflector.

${ }^{\mathrm{d}}$ Based on their Figure 4 (i.e., the $\log L_{\mathrm{X}}$ power-law parameterization for the absorption fraction, constant $N_{\mathrm{H}}$ distribution, and local type-2 to type-1 AGN ratio of 4.0 are assumed).

e Only a representative set of the parameters examined by them is shown here.

${ }^{\mathrm{f}}$ The first and second values correspond to those of type-1 and type-2 AGNs, respectively.

References. (1) Ueda et al. 2003; (2) Ballantyne et al. 2006; (3) Gilli et al. 2007; (4) Treister et al. 2009; (5) Akylas et al. 2012.

(2007) basically adopt the $0.5-2 \mathrm{keV}$ XLF of type-1 AGNs derived by $\mathrm{H} 05$ and determine a luminosity-dependent (but redshift independent) ratio between obscured and unobscured AGNs by fitting the data points of the 2-10 keV XLF obtained by U03 and La Franca et al. (2005). Ballantyne et al. (2006) and Treister et al. (2009) take into account the evolution of the absorption fraction. The scatter in the photon index distribution is considered in the Gilli et al. (2007) and Akylas et al. (2012) models. All these authors adopt slightly different values of the high energy cut-off and Compton reflection strength.

A validity of the models can be checked by the predicted XRB intensity. The Ballantyne et al. (2006) model significantly overproduces the XRB intensity at $25 \mathrm{keV}$ when compared with recent measurements by Swift/BAT and INTEGRAL. The same problem existing in the earlier model by Treister \& Urry (2005) is corrected in Treister et al. (2009), where a very small CTK AGN fraction $\left(f_{\text {СтK }}=0.17\right)$ is assumed on the basis of hard $\mathrm{X}$-ray $(>10 \mathrm{keV}$ ) surveys in the local universe. However, after correcting for biases against detecting heavily CTK AGNs even in the hard X-ray band, $10 \mathrm{keV}$, the intrinsic fraction of CTK AGNs could be much larger than the value assumed in Treister et al. (2009) (Section 3; see also Burlon et al. 2011).
Our model thus supersedes the older models and may be regarded as a standard population synthesis model of the XRB at the current stage. The biggest advantage is that it utilizes the most precise XLF and absorption function that depends both on luminosity and redshift. Our model also takes into account the broadband spectra, including the reflection components from the tori based on the "luminosity- and redshift-dependent unified scheme" as well as the photon index distributions that are different between type-1 and type-2 AGNs. The whole analysis has been performed self-consistently on the basis of these assumptions. Compared with Gilli et al. (2007), we predict a higher fraction of obscured and CTK AGNs at faint fluxes due to the inclusion of the redshift evolution in the absorption fraction (Figure 17). We note that Draper \& Ballantyne (2009) report a possible contribution of blazars to the XRB, which is ignored in our model. According to their model with an X-ray duty cycle of $13 \%$, the integrated emission of blazars can account for $\sim 2 \%$ of the XRB at $20 \mathrm{keV}$, which is much smaller than the current uncertainties in its absolute intensity. The estimate should be largely uncertain, however, as the model significantly overpredicts the blazar source counts obtained with Swift/BAT (Draper \& Ballantyne 2009). 


\section{BOLOMETRIC LUMINOSITY FUNCTION OF AGNs AND GROWTH HISTORY OF SMBH}

\subsection{Bolometric Luminosity Function}

The luminosity function of the whole AGN population provides a basis for understanding the growth history of SMBHs in galactic centers. While hard X-rays are an ideal energy band for a complete survey of AGNs with little contamination, they represent only a limited fraction of the total radiation energy emitted from an AGN, whose SED has a peak around the ultraviolet band. Thus, it is very convenient to determine the BLF of all AGNs (including both CTN and CTK AGNs) based on the XLF. The BLF is defined as a function of bolometric luminosity $L$ (instead of $L_{\mathrm{X}}$ ) and $z$ so that

$$
\frac{d \Phi_{\mathrm{bol}}(L, z)}{d \log L}
$$

gives the comoving spatial number density per $\log L$. Hopkins et al. (2007) derived a BLF of AGNs by simultaneously analyzing multiple AGN surveys performed in the X-ray, optical, and mid-infrared bands. In this section, we derive the AGN BLF directly from our new XLF by taking into account the luminosity dependence bolometric correction and its scatter that are estimated by Hopkins et al. (2007). The full revision of the work of Hopkins et al. (2007) by utilizing AGN multiband luminosity functions other than the XLF is beyond the scope of this paper. To combine multiwavelength data sets, it is crucial to evaluate the selection function against obscuration, which is not necessarily trivial given the complex situation of each survey.

We define the bolometric correction factor, $k \equiv L / L_{X}$, to convert from an X-ray luminosity into a bolometric one. According to Hopkins et al. (2007), its average $\bar{k}$ is a function of $L$ and is represented as

$$
\bar{k}(L)=10.83\left(\frac{L}{10^{10} L_{\odot}}\right)^{0.28}+6.08\left(\frac{L}{10^{10} L_{\odot}}\right)^{-0.020} .
$$

The standard deviation in $\log k$ is also given as a function of $L$;

$$
\sigma_{\log k}(L)=0.06\left(L / 10^{9} L_{\odot}\right)^{0.10}+0.08 .
$$

To determine the BLF from our data, we take an approximated approach instead of performing detailed calculations as done in Section 6. A BLF of all AGNs can be converted into the XLF of CTN AGNs by assuming that the logarithm of the bolometric correction factor has a Gaussian distribution:

$$
\begin{aligned}
\frac{d \Phi_{\mathrm{X}}^{\mathrm{CTN}}\left(L_{\mathrm{X}}, z\right)}{d \log L_{\mathrm{X}}}= & \int \frac{d \Phi_{\mathrm{bol}}(L, z)}{d \log L} \frac{1}{\sqrt{2 \pi} \sigma_{\log k}} \\
& \times \exp \left[-\frac{(\log (k / \bar{k}(L)))^{2}}{2 \sigma_{\log k}^{2}}\right] d \log k .
\end{aligned}
$$

Once the XLF is obtained, we calculate the predicted number of detectable AGNs in our surveys as a function of $L_{X}$ and $z$ by simply correcting for the ratio of the XLF value to the best-fit one presented in Section 6.2,

$$
N\left(L_{\mathrm{X}}, z\right)=N_{\text {best }}\left(L_{\mathrm{X}}, z\right) \frac{d \Phi_{\mathrm{X}}^{\mathrm{CTN}}\left(L_{\mathrm{X}}, z\right) / d \log L_{\mathrm{X}}}{d \Phi_{\mathrm{X}, \text { best }}^{\mathrm{CTN}}\left(L_{\mathrm{X}}, z\right) / d \log L_{\mathrm{X}}},
$$

which can be compared with the observed number of AGNs. By dividing the $L_{\mathrm{X}}$ and $z$ plane within the range of $L_{\mathrm{X}}=$
$10^{41}-10^{47}$ and $z=0.002-5.0$ into $120 \times 136$ logarithmic bins, respectively, we perform the Poisson ML fit to the binned data because the numbers of sources in each pixel are small, often less than 10. Here we adopt the same analytic form for the BLF as for the XLF by setting $\log L_{\mathrm{p}}=\log L_{\mathrm{a} 2}=$ 45.67, a bolometric luminosity that corresponds to $\log L_{\mathrm{X}}=$ 44 with the conversion given in Equation (21). In the fit, we fix $z_{\mathrm{c} 1}^{*}=1.86$ and $\alpha_{1}=0.29$, the best-fit values of the XLF, while the other parameters are left free. The resultant best-fit parameters of the BLF are summarized in Table 4. Figure 20 (left) plots the bolometric luminosity density (i.e., emissivity) of all AGNs $\int L\left(d \Phi_{\text {bol }}(L, z) / d \log L\right) d \log L$ as a function of redshift integrated in different luminosity ranges. For reference, those in the $2-10 \mathrm{keV}$ band based on the XLF are plotted in Figure 20 (left). In both figures, the integrated emissivity has a peak around $z \sim 2$, where AGNs with $\log L=46-47$ or $\log L_{\mathrm{X}}=44-45$ make the largest contribution. We note that the peak redshift of the integrated emissivity is significantly larger than $z \approx 1.2$ predicted from the LADE model by Aird et al. (2010, see their Figure 11). This reflects the fact that our LDDE model gives a larger number of luminous AGNs with $\log L_{\mathrm{X}} \gtrsim$ 44 than the LADE model at $z \gtrsim 1$.

\subsection{Evolution of Mass Function of SMBHs}

As mentioned in Section 1, an AGN is the process where an SMBH gains its mass by accretion, and hence the AGN luminosity function records the growth history of SMBHs. A bolometric luminosity $L$ can be related to the mass accretion rate onto an $\mathrm{SMBH}, \dot{M}_{\mathrm{acc}}$, as $L=\eta \dot{M}_{\mathrm{acc}} c^{2}$, where $\eta$ is the mass-to-energy conversion factor (or radiation efficiency). The $\eta$ value is predicted to be 0.054 for a standard disk around a non-rotating black hole and becomes as large as 0.42 for that with a maximum spin. In radiatively inefficient accretion flows (RIAFs), it could be significantly smaller. Hence, in general, the averaged value of $\eta$ could depend on parameters like black hole mass $M$ and $z$.

On the basis of Soltan's argument (Soltan 1982), one can estimate the total mass density of SMBHs $\rho(z)$ as a function of redshift once the BLF of AGN is known by using the following equation:

$$
\rho(z)=\rho\left(z_{\mathrm{s}}\right)+\frac{1-\bar{\eta}}{\bar{\eta} c^{2}} \int_{z}^{z_{\mathrm{s}}} d z \frac{d t}{d z} \int_{L_{\min }}^{L_{\max }} L \frac{d \Phi_{\mathrm{bol}}(L, z)}{d \log L} d \log L .
$$

Here, $\rho\left(z_{\mathrm{s}}\right)$ gives the initial mass density at $z=z_{\mathrm{s}}$ from which the time integration starts, and $\bar{\eta}$ represents an averaged radiation efficiency, which is assumed to be independent of $z$ and $L$. A detailed calculation using our model indicates that $\approx 74 \%$ $(\approx 37 \%)$ of the total energy emitted by whole AGNs in the history of the universe (hence, the total mass of all SMBHs at $z=0$ ) was produced by obscured accretion with $\log N_{\mathrm{H}}=$ 22-26 $\left(\log N_{\mathrm{H}}=24-26\right)$. The mass density of SMBHs in the local universe can be independently estimated from the empirical relation between SMBH mass and host-spheroid luminosity (or mass). For instance, if we adopt the result by Vika et al. (2009), $\rho^{\text {obs }}(z=0)=(4.9 \pm 0.7) \times 10^{5} M_{\odot} \mathrm{Mpc}^{-3}$, $\bar{\eta}=0.080_{-0.009}^{+0.013}$ is suggested. This confirms earlier works based on the hard XLF of AGNs (e.g., Marconi et al. 2004; Li et al. 2012). As described below, however, Kormendy \& Ho (2013) have recently reported that the SMBH masses of classical bulges and elliptical galaxies should be revised upward by a factor of $\sim 2-4$, which would lead to a reduction of $\bar{\eta}$ by a similar factor (see also Novak 2014). Figure 21 plots the results calculated 

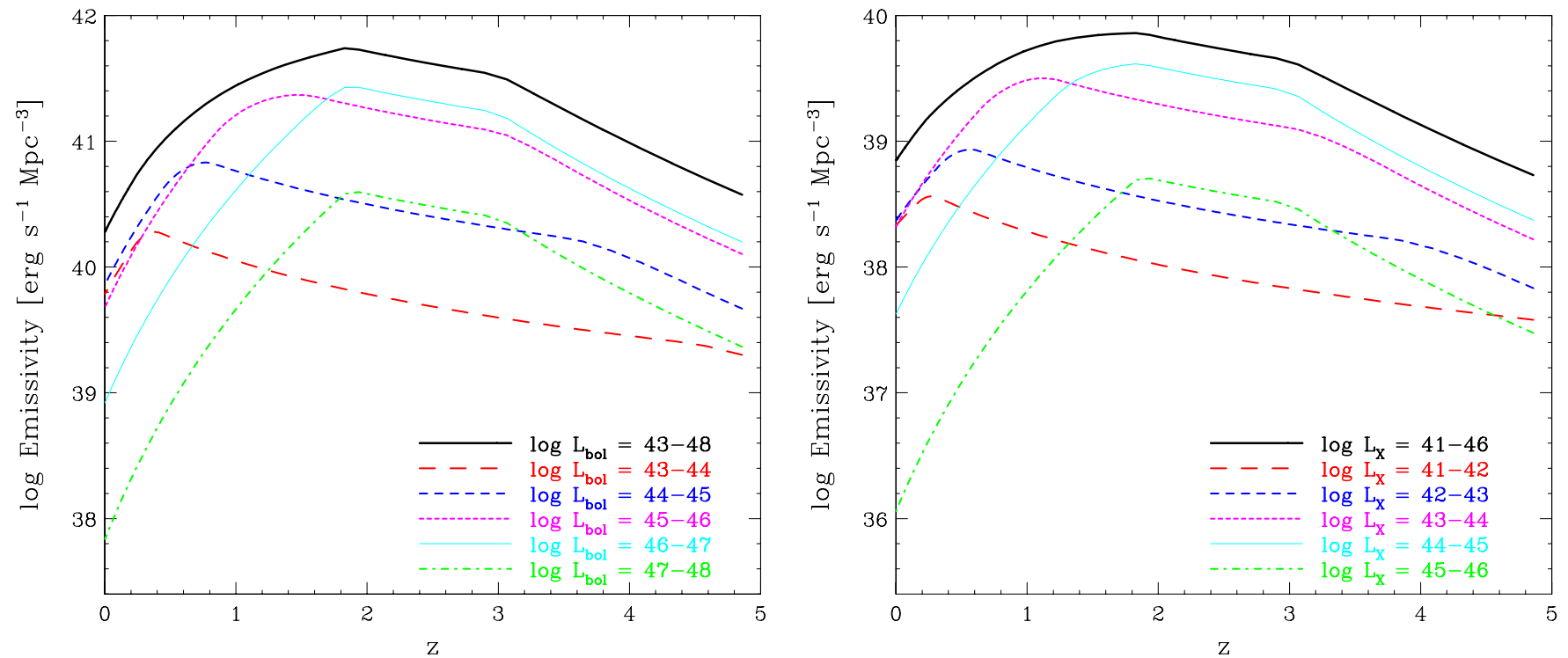

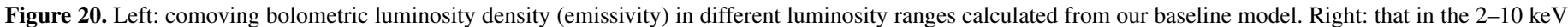
band.

(A color version of this figure is available in the online journal.)

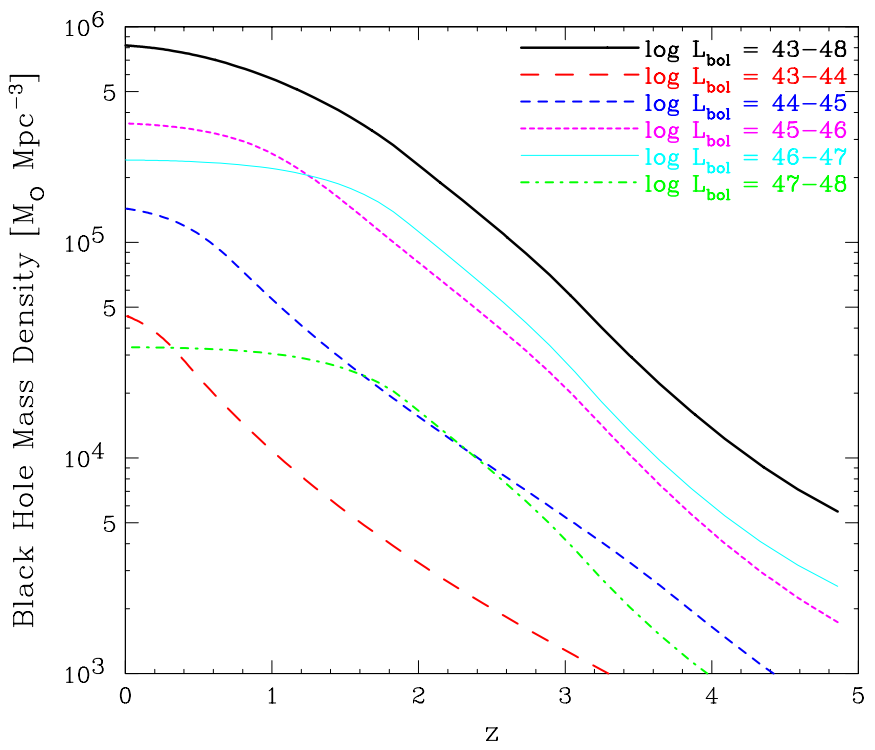

Figure 21. Comoving mass density of all SMBHs plotted against redshift (uppermost solid curve, black). Those calculated within limited luminosity ranges are separately shown. The averaged radiation efficiency of $\bar{\eta}=0.05$ is assumed.

(A color version of this figure is available in the online journal.)

with $\bar{\eta}=0.05$ in different luminosity ranges, $\log L=43-48$, 43-44, 44-45, 45-46, 46-47, and 47-48. We adopt $z_{\mathrm{s}}=5$ and estimate $\rho\left(z_{\mathrm{s}}\right)$ by assuming that all SMBHs were AGNs with a mean Eddington ratio of $10^{-1.1}$ (see below).

As studied by many authors (e.g., Small \& Blandford 1992; Yu \& Tremeine 2012; Marconi et al. 2004; Shankar et al. 2004, 2009, 2013; Tamura et al. 2006; Cao \& Li 2008; Li et al. 2012), it is also possible to trace the cosmological evolution of the mass function (MF) of all SMBHs, including both active (i.e., AGNs) and non-active ones, from the AGN luminosity function ("AGN relic MF"). Here, we define the MF of all SMBHs and that of only AGNs as $N(z, M)$ and $N_{\mathrm{AGN}}(z, M)$, respectively, which represent their comoving spatial number density per unit mass at redshift $z$. The Eddington ratio is given as $\lambda \equiv L / L_{\text {Edd }}$, where $L_{\mathrm{Edd}}=1.25 \times 10^{38}\left(M / M_{\odot}\right) \mathrm{erg} \mathrm{s}^{-1}$ is the Eddington limit for a mass of $M$. Under the assumption that the merging of SMBHs can be ignored, we can introduce the continuity equation of the MF of all SMBHs (e.g., Small \& Blandford 1992),

$$
\begin{aligned}
& \frac{\partial N(z, M)}{\partial z} \frac{d z}{d t}=-\frac{\partial}{\partial M}[N(z, M)\langle\dot{M}\rangle] \\
& =-\frac{\partial}{\partial M}\left[\frac{1-\eta(z, M)}{\eta(z, M)} \frac{\bar{\lambda}(z, M) L_{\mathrm{Edd}} N_{\mathrm{AGN}}(z, M)}{c^{2}}\right],
\end{aligned}
$$

where $\langle\dot{M}\rangle$ is the averaged black hole growth rate of all SMBHs, and $\bar{\lambda}(z, M)$ gives the averaged Eddington ratio of $A G N s$ with a mass of $M$ at redshift $z$. The MF of AGNs can be calculated as

$$
N_{\mathrm{AGN}}(z, M)=\frac{d \log M}{d M} \int \frac{d \Phi_{\mathrm{bol}}(z, L)}{d \log L} P(\lambda \mid L, z) d \log \lambda,
$$

where $P(\lambda \mid L, z)$ is the Eddington ratio distribution function per unit $\log \lambda$ at a given luminosity $L$ and redshift $z$. The averaged AGN Eddington ratio is then given as

$$
\bar{\lambda}(z, M)=\int \lambda \frac{\Phi_{\mathrm{bol}}(z, L)}{N_{\mathrm{AGN}}(z, M)} P(\lambda \mid L, z) d \log \lambda .
$$

Following Li et al. (2012), we assume that the Eddington ratio distribution function is log-normal,

$$
P(\lambda \mid L, z)=\frac{1}{\sqrt{2 \pi} \sigma_{\log \lambda}} \exp \left[-\frac{(\log (\lambda / \bar{\lambda}))^{2}}{2 \sigma_{\log \lambda}^{2}}\right]
$$

and is independent of luminosity and redshift.

Marconi et al. (2004) consider the simplest case where $\eta(M, z)$ is constant and $P(\lambda \mid L, z)$ is a delta function at $\bar{\lambda}$ (i.e., $\sigma_{\log \lambda}=0.0$ ). Using the BLF converted from the U03 XLF, they find that $\eta \sim 0.1$ and $\lambda \sim 1.0$ to explain the observed MF of all 

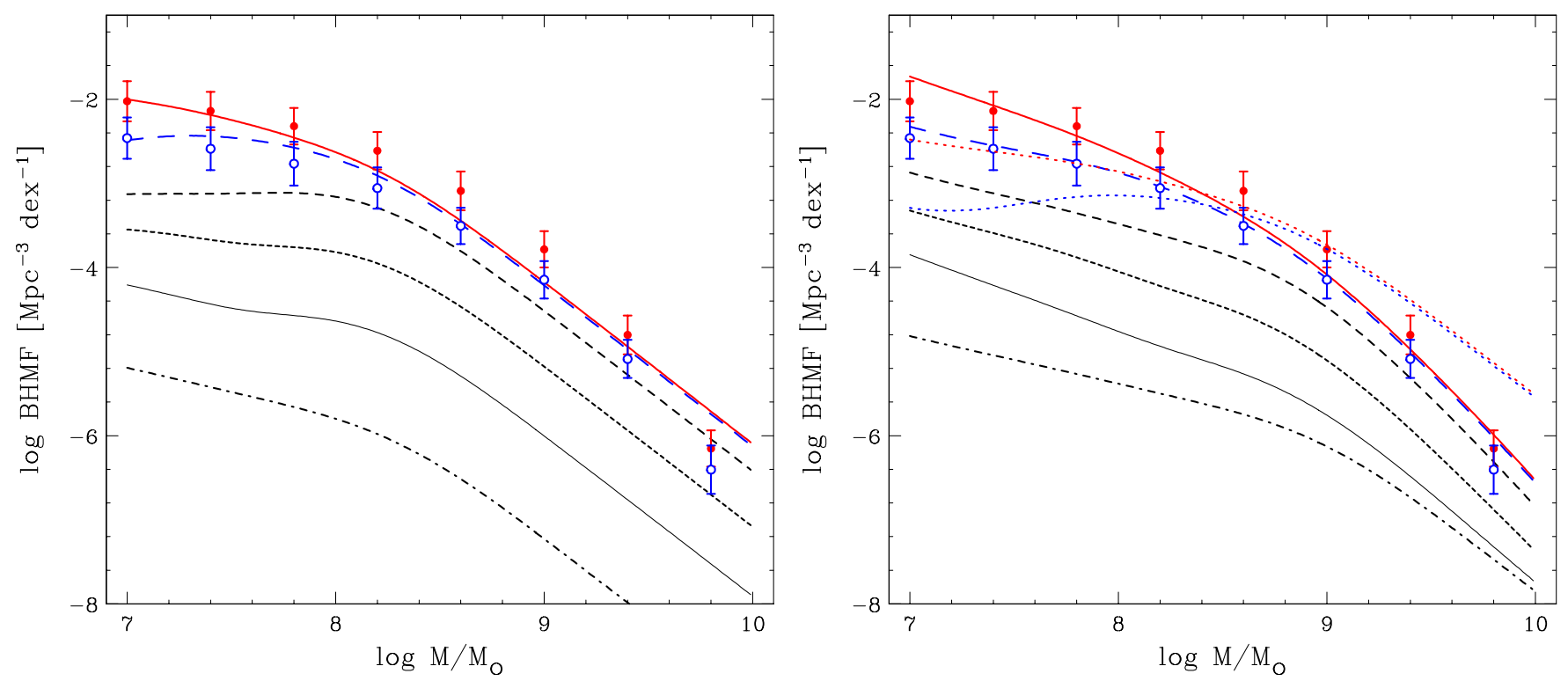

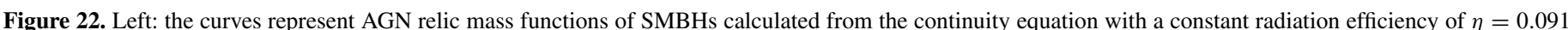

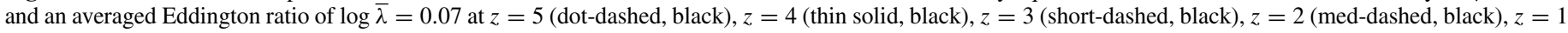

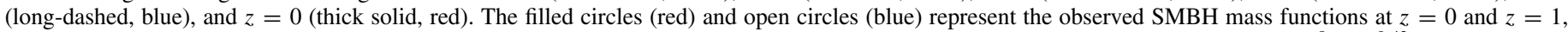

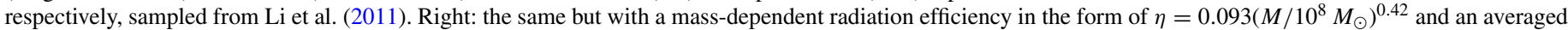

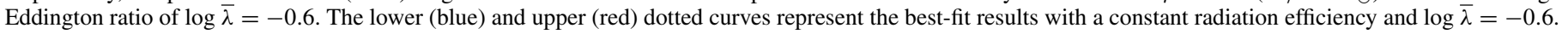

(A color version of this figure is available in the online journal.)

SMBHs at $z=0$ with the AGN relic MF. Tamura et al. (2006) show that the SMBH MFs at several redshifts of $z=0-1.05$ derived from early-type galaxy luminosity functions are broadly consistent with the AGN relic ones calculated with $\eta=0.1$ and $\lambda=1.0$.

In a similar way, we first assume that the radiation efficiency does not depend on black hole mass and redshift. We calculate AGN relic MFs based on the new AGN BLF derived above, and compare them with the observed SMBH MFs at $z=0$ and $z=1$ estimated by Li et al. (2011). For each redshift, we take eight discrete data points of $\log N(z, M)$, equally separated in a range of $\log \left(M / M_{\odot}\right)=7.0-9.6$, and attach an effective error to each point by the half difference between the minimum and maximum allowed values indicated in Figure 4 of $\mathrm{Li}$ et al. (2011). We make the radiation efficiency $\eta$ and the averaged Eddington ratio $\bar{\lambda}$ free parameters, and fix $\sigma_{\log \lambda}=0.3$. The initial MF is calculated $z=5$ from the BLF at the same redshift by assuming that all SMBHs were shining (i.e., AGNs) with a constant Eddington ratio of $\bar{\lambda}$. Fitting the AGN relic MFs simultaneously to the data points at $z=0$ and $z=1$ with the $\chi^{2}$ algorithm, we obtain $\eta=0.091_{-0.016}^{+0.019}$ and $\log \bar{\lambda}=0.07 \pm 0.08$ with $\chi^{2} /$ dof $=18.7 / 14$ (the errors are $1 \sigma$ confidence limits). These values are consistent with the previous result by Marconi et al. (2004) within the errors. The resultant AGN relic MFs at $z=5,4,3,2,1$, and 0 are plotted in Figure 22 (left), compared with the data points of the observed SMBH MFs at $z=0$ and $z=1$.

Studies based on black hole mass measurements of optical (e.g., Kollmeier et al. 2006; Kelly \& Shen 2013) and X-ray (e.g., Nobuta et al. 2012) selected AGN samples indicate, however, that their averaged Eddington ratio is significantly smaller than $\lambda=1$. This suggests that the apparently good reproduction of the SMBH MF by assuming constant $\lambda \simeq 1$ and $\eta \simeq 0.1$ would not represent the actual case. A solution to solve this contradiction is to introduce the mass dependence of the radiation efficiency, as pointed out by Cao \& $\mathrm{Li}$ (2008). More recently, $\mathrm{Li}$ et al. (2012) constrained the radiation efficiency as a function of both $z$ and $M$; they find that $\eta(z, M)$ is roughly proportional to $M^{0.5}$ at $z>1$, confirming the trend reported by Cao \& $\mathrm{Li}$ (2008), while the mass dependence becomes weaker or even inverted at lower redshifts.

Accordingly, we empirically model $\eta$ as a power-law function of black hole mass,

$$
\eta(z, M)=\eta_{8}\left(M / 10^{8} M_{\odot}\right)^{\delta},
$$

although here we ignore the redshift dependence for simplicity. We adopt $\log \bar{\lambda}=-0.6$ and $\sigma_{\log \lambda}=0.3$ (Kollmeier et al. 2006), as done in Li et al. (2012). Then, performing a $\chi^{2}$ fit to the observed SMBH MFs at $z=0$ and $z=1$ by Li et al. (2011), we obtain $\eta_{8}=0.093_{-0.010}^{+0.012}$ and $\delta=0.42 \pm 0.05$ with $\chi^{2} /$ dof $=10.3 / 14$. Figure 22 (right) plots the predicted AGN relic MFs at several redshifts, which are in very good agreement with the observed ones (data points). If the mass dependence of $\eta$ is ignored (i.e., $\delta=0$ ), the AGN relic MFs significantly underestimate (overestimate) the observed MFs at mass ranges lower (higher) than $\log M \approx 8$ at both $z=1$ and $z=0$; we plot this case by the dotted lines in Figure 22 (right). The large discrepancy at $z=0$ is attributable to that already present at $z=1$, suggesting that the assumption of constant $\eta(z, M)$ is not proper at $z>1$, unlike the case of $\bar{\lambda} \simeq 1.0$ discussed earlier.

Recently, Kormendy \& Ho (2013) have updated the calibration between SMBH mass and the luminosity, mass, or velocity dispersion of the bulge component of the host galaxy in the local universe. This leads to an upward revision by a factor of $\sim 2-4$ of SMBH masses that have been previously used. To examine the influences by this revision, we update the SMBH MFs of Li et al. (2011), by assuming the new SMBH-mass versus bulge-mass relation given as Equation (10) of Kormendy \& Ho (2013), instead of that of Häring \& Rix (2004) adopted by Li et al. (2011). The revision of the SMBH masses also affects the deviation of the Eddington-ratio distribution of AGNs. We find that the Eddington ratios in Kollmeier et al. (2006) should be decreased 

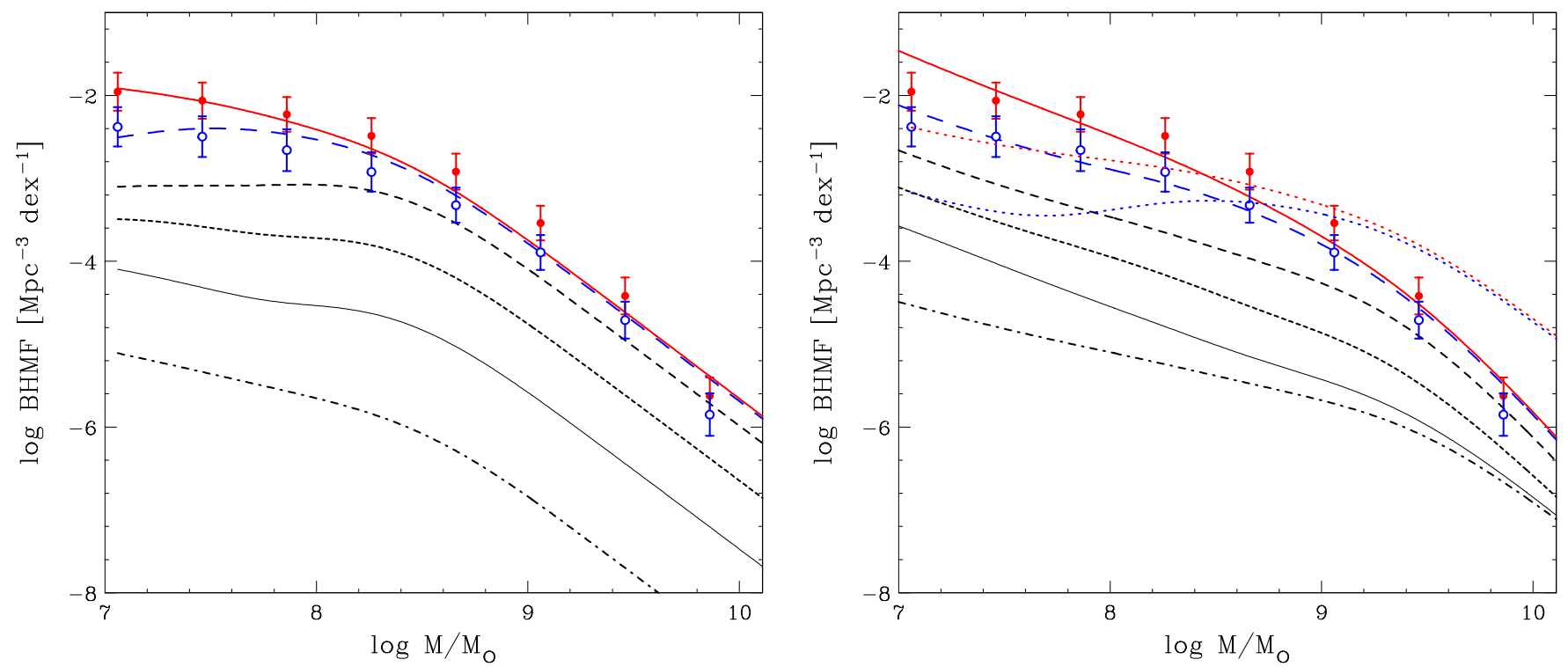

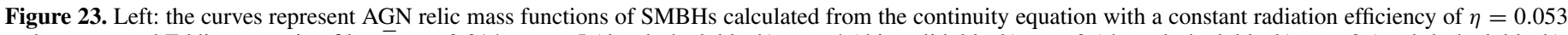

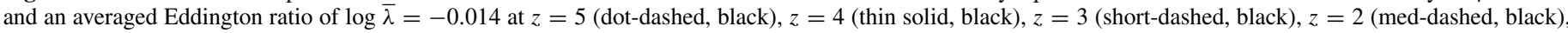

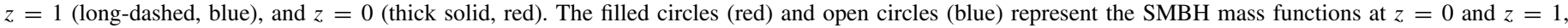

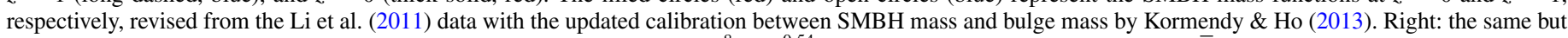

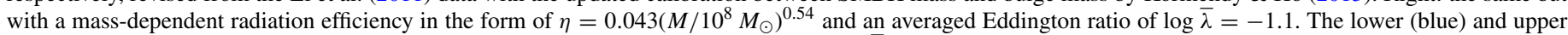
(red) dotted curves represent the best-fit results with a constant radiation efficiency and $\log \bar{\lambda}=-1.1$.

(A color version of this figure is available in the online journal.)

by a factor of $\approx 3$ when we refer to Equation (3) of Kormendy $\&$ Ho (2013), yielding an updated mean value of $\log \lambda \approx-1.1$. We then repeat the same analysis as above by fitting AGN relic MFs to these revised SMBH MFs. The results are shown in Figure 23. When a constant radiation efficiency is assumed, we obtain $\eta=0.053_{-0.006}^{+0.008}$ and $\log \bar{\lambda}=-0.14 \pm 0.07$ with $\chi^{2} /$ dof $=11.2 / 14$ (Figure 23 (left)). This Eddington ratio is significantly larger than the observed value $(\log \lambda=-1.1)$. When we fix $\bar{\lambda}=-1.1$ and $\sigma_{\log \lambda}=0.3$, the AGN relic MFs cannot reproduce the observed MFs with $\chi^{2} /$ dof $=140 / 15$. Introducing a power-law-like mass dependence of the radiation efficiency again significantly improves the fit, giving $\eta_{8}=0.043 \pm 0.006$ and $\delta=0.54 \pm 0.05$ with $\chi^{2} /$ dof $=13.7 / 14$ (Figure 23 (right)).

Thus, these arguments based on the new AGN BLF and updated SMBH MFs are consistent with those by Cao \& $\mathrm{Li}$ (2008), Li et al. (2012), and Shankar et al. (2013) that the radiation efficiency should increase with black hole mass, at least at $z>1$. The possible contribution of mergers neglected here only works to increase the predicted MF at the high mass region, and hence does not essentially change this conclusion (see the discussion of Shankar et al. 2013). Importantly, we find that the inferred radiation efficiency could be significantly reduced compared with the previous estimates by the revision of the SMBH MFs. Our results imply that in relatively lowmass (hence low-luminosity) AGNs with $\log \left(M / M_{\odot}\right) \lesssim 8.2$, where $\eta<0.054$ on average, the standard accretion disk would be truncated before reaching the radius corresponding to the innermost stable circular orbit of a non-rotating black hole and is replaced by a RIAF. The inferred high radiation efficiencies of higher mass AGNs suggest that their SMBHs are rotating, implying that they grew predominantly by accretion.

We note, however, that the exact results depend on the assumption of the Eddington ratio distribution function that is constant against luminosity and redshift in the above analysis. Recent work using the X-ray selected AGNs at $z \sim 1.4$ in the SXDS field by Nobuta et al. (2012) suggests that the mean
Eddington ratio is smaller at lower luminosities. Furthermore, no consensus has been established on its possible redshift evolution. To obtain robust conclusions, it is important to determine the Eddington ratio distribution function and the MF of AGNs over a wide range of luminosity and redshift based on accurate determination of their black hole masses.

\section{CONCLUSIONS}

1. We have compiled so far the largest, highly complete sample of AGNs detected in X-ray surveys performed with Swift/ BAT, MAXI, ASCA, XMM-Newton, Chandra, and ROSAT, consisting of 4039 detections in the soft $(0.5-2 \mathrm{keV})$ and/or hard $(>2 \mathrm{keV})$ band. This gives us the best opportunity to trace the cosmological evolution of absorption properties and XLF of all AGNs with $\log L_{\mathrm{X}}(2-10 \mathrm{keV})=42-46$, including both type-1 (unabsorbed) and type-2 (absorbed) ones, from $z=0$ to $z=5$.

2. Using the latest compilation of spectral analysis of individual AGNs detected in the Swift/BAT survey, we determine the shape of the absorption $\left(N_{\mathrm{H}}\right)$ function in the local universe. We find that the fraction of absorbed AGNs with $\log N_{\mathrm{H}}=22-24$ among all CTN AGNs with $\log N_{\mathrm{H}} \leqslant 24$ is $0.43 \pm 0.03$ at $\log L_{\mathrm{X}}=43.75$. The distribution of the photon index is peaked at $\overline{\Gamma_{1}}=1.94 \pm 0.03$ for type- 1 AGNs and $\overline{\Gamma_{2}}=1.84 \pm 0.04$ for type-2 AGNs.

3. We confirm that the absorbed fraction of AGNs increases toward higher redshifts by keeping the anti-correlation with the luminosity. At $\log L_{\mathrm{X}}=43.75$, the fraction of AGNs with $\log N_{\mathrm{H}}=22-24$ among those with $\log N_{\mathrm{H}} \leqslant 24$ is proportional to $(1+z)^{0.48 \pm 0.05}$ up to $z=2$.

4. To constrain the XLF of AGNs, we have developed a novel analysis method where we perform a ML fit directly to the list of the observed count rate and redshift by taking into account selection biases in each survey. Here, we consider the evolution of the absorbed fraction, the contribution of 
CTK AGNs with $\log N_{\mathrm{H}} 24-26$, and AGN broadband X-ray spectra, including reflection components from tori based on the luminosity- and redshift-dependent unified scheme.

5. We find that the shape of the XLF at $z \sim 1-3$ is significantly different from that in the local universe, showing flatter slopes in the lower luminosity range below the break. Its cosmological evolution is well described with the LDDE model, while the LADE model fails to fit the data.

6. On the basis of the absorption function and XLF determined above, we have newly constructed a population synthesis model of the XRB, which can be regarded as a "standard model" which well reproduces the source counts in both soft and hard bands, the observed fractions of CTK AGNs, and the spectrum of the hard XRB.

7. To reproduce the hard XRB intensity in the $20-50 \mathrm{keV}$ band within current uncertainties, we constrain that the fraction of CTK AGNs with $\log N_{\mathrm{H}}=24-26$ to absorbed CTN AGNs with $\log N_{\mathrm{H}}=22-24$ should be $0.5-1.6$. This is also well consistent with the results of hard X-ray surveys above $\gtrsim 10 \mathrm{keV}$ currently available.

8. We determine a BLF of AGNs by considering the luminosity-dependent bolometric correction factor and its variation from the XLF. The luminosity density of the whole AGNs has a peak around $z \sim 2$, where AGNs with bolometric luminosities of $\log L=46-47$ make the largest contribution. On the basis of Soltan's argument, the most recent estimate of the local mass density of SMBHs is reproduced by adopting an averaged AGN radiation efficiency of $\approx 0.05$, although its mass dependence is suggested from the comparison of the AGN relic MF and observed ones at $z=0$ and $z=1$.

We thank Murray Brightman for his help on the torus model, Marco Ajello for providing the data of the XRB spectra in a machine readable form, David Alexander for sending the area curve of the CDFN survey, Bret Lehmer for his comments on the $\log N-\log S$ relations in the CDFS, Yan-Rong Li for sending the data of the black hole mass function, and John Kormendy, Francesco Shankar, Akylas Thanassis, Andy Strong, Richard Mushotzky, Chris Done, David Ballantyne, Claudio Ricci, Agnese Del Moro, John Silverman, Tohru Nagao, and Yuichi Terashima for discussions. We acknowledge the efforts by the SXDS team that have helped the identification work of the $\mathrm{X}$-ray sample. This work was partly supported by the Grant-inAid for Scientific Research 23540265 (Y.U.). T.M. acknowledges support from UNAM-DGAPA grant PAPIIT IN104113 and CONACyT grant Científica Básica 179662.

\section{REFERENCES}

Aird, J., Nandra, K., Laird, E. S., et al. 2010, MNRAS, 401, 2531

Ajello, M., Greiner, J., Sato, G., et al. 2008, ApJ, 689, 666

Akiyama, M., Ohta, K., Yamada, T., et al. 2000, ApJ, 532, 700

Akiyama, M., Ueda, Y., Ohta, K., Takahashi, T., \& Yamada, T. 2003, ApJS, 148,275

Akylas, A., Georgakakis, A., Georgantopoulos, I., Brightman, M., \& Nandra, K. 2012, A\&A, 546, 98

Alexander, D. M., Bauer, F. E., Brandt, W. N., et al. 2003, AJ, 126, 539 Alexander, D. M., Bauer, F. E., Brandt, W. N., et al. 2011, ApJ, 738, 44 Alexander, D. M., Stern, D., Del Moro, A., et al. 2013, ApJ, 773, 125 Appenzeller, I., Thiering, I., Zickgraf, F.-J., et al. 1998, ApJS, 117, 319 Baldi, A., Molendi, S., Comastri, A., et al. 2002, ApJ, 564, 190 Ballantyne, D. R. 2014, MNRAS, 437, 2845

Ballantyne, D. R., Everett, J. E., \& Murray, N. 2006, ApJ, 639, 740 Barcons, X., Carrera, F. J., Ceballos, M. T., et al. 2007, A\&A, 476, 1191 Barcons, X., Mateos, S., \& Ceballos, M. T. 2000, MNRAS, 316, L13
Barger, A. J., Cowie, L. L., Capak, P., et al. 2003, AJ, 126, 632

Barger, A. J., Cowie, L. L., Mushotzky, R. F., et al. 2005, AJ, 129, 578

Beckmann, V., Soldi, S., Ricci, C., et al. 2009, A\&A, 505, 417

Boyle, B. J., Griffiths, R. E., Shanks, T., Stewart, G. C., \& Georgantopoulos, I. 1993, MNRAS, 260, 49

Brandt, W. N., \& Hasinger, G. 2005, ARA\&A, 43, 827

Brightman, M., \& Nandra, K. 2011a, MNRAS, 413, 1206

Brightman, M., \& Ueda, Y. 2012, MNRAS, 423, 702

Brunner, H., Cappelluti, N., Hasinger, G., et al. 2008, A\&A, 479, 283

Brusa, M., Comastri, A., Gilli, R., et al. 2009, ApJ, 693, 8

Burlon, D., Ajello, M., Greiner, J., et al. 2011, ApJ, 728, 58

Cao, X.-W., \& Li, F. 2008, MNRAS, 390, 561

Chapman, S. C., Blain, A. W., Smail, I., \& Ivison, R. J. 2005, ApJ, 622, 772

Churazov, E., Sunyaev, R., Revnivtsev, M., et al. 2007, A\&A, 467, 529

Civano, F., Brusa, M., Comastri, A., et al. 2011, ApJ, 741, 91

Cocchia, F., Fiore, F., Vignali, C., et al. 2007, A\&A, 466, 31

Comastri, A., Setti, G., Zamorani, G., \& Hasinger, G. 1995, A\&A, 296, 1

Cowie, L. L., Songaila, A., Hu, E. M., \& Cohen, J. G. 1996, AJ, 112, 839

Daddi, E., Alexander, D. M., Dickinson, M., et al. 2007, ApJ, 670, 173

Dadina, M. 2008, A\&A, 485, 417

Della Ceca, R., Caccianiga, A., Severgnini, P., et al. 2008, A\&A, 487, 119

Della Ceca, R., Maccacaro, T., Caccianiga, A., et al. 2004, A\&A, 428, 383

De Luca, A., \& Molendi, S. 2004, A\&A, 419, 837

Draper, A. R., \& Ballantyne, D. R. 2009, ApJ, 707, 778

Ebrero, J., Carrera, F. J., Page, M. J., et al. 2009, A\&A, 493, 55

Eckart, M. E., Stern, D., Helfand, D. J., et al. 2006, ApJS, 165, 19

Eguchi, S., Ueda, Y., Awaki, H., et al. 2011, ApJ, 729, 31

Eguchi, S., Ueda, Y., Terashima, Y., Mushotzky, R., \& Tueller, J. 2009, ApJ, 696,1657

Ferrarese, L., \& Merritt, D. 2000, ApJL, 539, L9

Fiore, F., Brusa, M., Cocchia, F., et al. 2003, A\&A, 409, 79

Fiore, F., Grazian, A., Santini, P., et al. 2008, ApJ, 672, 94

Fiore, F., Puccetti, S., Brusa, M., et al. 2009, ApJ, 693, 447

Fiore, F., Puccetti, S., Grazian, A., et al. 2012, A\&A, 537, 16

Fontanot, F., De Lucia, G., Monaco, P., Somerville, R. S., \& Santini, P. 2009, MNRAS, 397, 1776

Fotopoulou, S., Salvato, M., Hasinger, G., et al. 2012, ApJS, 198, 1

Frontera, F., Orlandini, M., Landi, R., et al. 2007, ApJ, 666, 86

Fukazawa, Y., Hiragi, K., Mizuno, M., et al. 2011, ApJ, 727, 19

Furusawa, H., Kosugi, G., Akiyama, M., et al. 2008, ApJS, 176, 1

Gebhardt, K., Bender, R., Bower, G., et al. 2000, ApJL, 539, L13

Gehrels, N. 1986, ApJ, 303, 336

Gendreau, K. C., Mushotzky, R., Fabian, A. C., et al. 1995, PASJ, 47, L5

Gilli, R., Comastri, A., \& Hasinger, G. 2007, A\&A, 463, 79

Gilli, R., Risaliti, G., \& Salvati, M. 1999, A\&A, 347, 424

Gioia, I. M., Henry, J. P., Mullis, C. R., et al. 2003, ApJS, 149, 29

Grossan, B. 1992, PhD thesis, MIT

Gruber, D. E., Matteson, J. L., Peterson, L. E., \& Jung, G. V. 1999, ApJ, 520,124

Gültekin, K., Richstone, D. O., Gebhardt, K., et al. 2009, ApJ, 698, 198

Häring, N., \& Rix, H. 2004, ApJL, 604, L89

Hasinger, G. 2008, A\&A, 490, 905

Hasinger, G., Altieri, B., Arnaud, M., et al. 2001, A\&A, 365, L45

Hasinger, G., Cappelluti, N., Brunner, H., et al. 2007, ApJS, 172, 29

Hasinger, G., Miyaji, T., \& Schmidt, M. 2005, A\&A, 441, 417 (H05)

Hiroi, K., Ueda, Y., Isobe, N., et al. 2011, PASJ, 63S, 677

Hiroi, K., Ueda, Y., Akiyama, M., \& Watson, M. G. 2012, ApJ, 758, 49

Hopkins, P. F., Hernquist, L., Cox, T. J., Robertson, B., \& Krause, E. 2007, ApJ, 669,67

Ichikawa, K., Ueda, Y., Terashima, Y., et al. 2012, ApJ, 754, 45

Ikeda, S., Awaki, H., \& Terashima, Y. 2009, ApJ, 692, 608

Iwasawa, K., Gilli, R., Vignali, C., et al. 2012, A\&A, 546, 84

Iwasawa, K., \& Taniguchi, Y. 1993, ApJL, 413, L15

Jones, L. R., McHardy, I. M., Merrifield, M. R., et al. 1997, MNRAS, 285, 547

Kelly, B. C., \& Shen, Y. 2013, ApJ, 764, 45

Kodama, T., Yamada, T., Akiyama, M., et al. 2004, MNRAS, 350, 1005

Kollmeier, J. A., Onken, C. A., Kochanek, C. S., et al. 2006, ApJ, 648, 128

Kormendy, J., \& Bender, R. 2009, ApJL, 691, L142

Kormendy, J., \& Ho, L. C. 2013, ARA\&A, 51, 511

La Franca, F., Fiore, F., Comastri, A., et al. 2005, ApJ, 635, 864

Lehmer, B. D., Alexander, D. M., Bauer, F. E., et al. 2010, ApJ, 724, 559

Lehmer, B. D., Xue, Y. Q., Brandt, W. N., et al. 2012, ApJ, 752, 46

Li, Y.-R., Ho, L. C., \& Wang, J.-M. 2011, ApJ, 742, 33

Li, Y.-R., Wang, J.-M., \& Ho, L. C. 2012, ApJ, 749, 187

Maccacaro, T., della Ceca, R., Gioia, I. M., et al. 1991, ApJ, 374, 117

Magdziarz, P., \& Zdziarski, A. A. 1995, MNRAS, 273, 837

Magorrian, J., Tremaine, S., Richstone, D., et al. 1998, AJ, 115, 2285 
Maiolino, R., Mignoli, M., Pozzetti, L., et al. 2006, A\&A, 445, 457 Malizia, A., Stephen, J. B., Bassani, L., et al. 2009, MNRAS, 399, 944 Marconi, A., \& Hunt, L. K. 2003, ApJL, 589, L21

Marconi, A., Risaliti, G., Gilli, R., et al. 2004, MNRAS, 351, 169 Marshall, F. E., Boldt, E. A., Holt, S. S., et al. 1980, ApJ, 1980, 235, 4 Mason, K. O., Carrera, F. J., Hasinger, G., et al. 2000, MNRAS, 311, 456

Mateos, S., Barcons, X., Carrera, F. J., et al. 2005, A\&A, 444, 79

Matsuoka, M., Kawasaki, K., Ueno, S., et al. 2009, PASJ, 61, 999

Mignoli, M., Pozzetti, L., Comastri, A., et al. 2004, A\&A, 418, 827

Miyaji, T., Hasinger, G., \& Schmidt, M. 2000, A\&A, 353, 25

Miyaji, T., Hasinger, G., \& Schmidt, M. 2001, A\&A, 369, 49

Mullis, C. R., Henry, J. P., Gioia, I. M., et al. 2004, ApJ, 617, 192

Nobuta, K., Akiyama, M., Ueda, Y., et al. 2012, ApJ, 761, 143

Novak, G. S. 2014, MNRAS, submitted (arXiv:1310.3833)

Page, M. J., Mason, K. O., McHardy, I. M., Jones, L. R., \& Carrera, F. J. 1997, MNRAS, 291, 324

Paolillo, M., Schreier, E. J., Giacconi, R., Koekemoer, A. M., \& Grogin, N. A. 2004, ApJ, 611, 93

Perola, G. C., Puccetti, S., Fiore, F., et al. 2004, A\&A, 421, 491

Persic, M., \& Rephaeli, Y. 2002, A\&A, 382, 843

Piccinotti, G., Mushotzky, R. F., Boldt, E. A., et al. 1982, ApJ, 253, 485

Reddy, N. A., Steidel, C. C., Erb, D. K., Shapley, A. E., \& Pettini, M. 2006, ApJ, 653, 1004

Ricci, C., Paltani, S., Awaki, H., et al. 2013, A\&A, 553, 29

Risaliti, G., Maiolino, R., \& Salvati, M. 1999, ApJ, 522, 157

Schwope, A., Hasinger, G., Lehmann, I., et al. 2000, AN, 321, 1

Scoville, N., Aussel, H., Brusa, M., et al. 2007, ApJS, 172, 1

Shinozaki, K., Miyaji, T., Ishisaki, Y., Ueda, Y., \& Ogasaka, Y. 2006, AJ, 131,2843

Silverman, J. D., Green, P. J., Barkhouse, W. A., et al. 2005, ApJ, 624, 630

Silverman, J. D., Green, P. J., Barkhouse, W. A., et al. 2008, ApJ, 679, 118

Shankar, F., Salucci, P., Granato, G. L., De Zotti, G., \& Danese, L. 2004, MNRAS, 354, 1020

Shankar, F., Weinberg, D. H., \& Miralda-Escudé, J. 2009, ApJ, 690, 20

Shankar, F., Weinberg, D. H., \& Miralda-Escudé, J. 2013, MNRAS, 428, 421

Simpson, C. 2005, MNRAS, 360, 565

Small, T. A., \& Blandford, R. D. 1992, MNRAS, 259, 725

Soltan, A. 1982, MNRAS, 200, 115
Steffen, A. T., Barger, A. J., Capak, P., et al. 2004, AJ, 128, 1483

Steffen, A. T., Barger, A. J., Cowie, L. L., Mushotzky, R. F., \& Yang, Y. 2003, ApJL, 596, L23

Swinbank, A. M., Smail, I., Chapman, S. C., et al. 2004, ApJ, 617, 64

Tamura, N., Ohta, K., \& Ueda, Y. 2006, MNRAS, 365, 134

Tozzi, P., Gilli, R., Mainieri, V., et al. 2006, A\&A, 451, 457

Treister, E., \& Urry, C. M. 2005, ApJ, 630, 115

Treister, E., \& Urry, C. M. 2006, ApJL, 652, L79

Treister, E., Urry, C. M., \& Virani, S. 2009, ApJ, 696, 110

Trouille, L., Barger, A. J., Cowie, L. L., Yang, Y., \& Mushotzky, R. F. 2008, ApJS, 179, 1

Trouille, L., Barger, A. J., Cowie, L. L., Yang, Y., \& Mushotzky, R. F. 2009, ApJ, 703, 2160

Tueller, J., Mushotzky, R. F., Barthelmy, S., et al. 2008, ApJ, 681, 113

Ueda, Y., Akiyama, M., Ohta, K., \& Miyaji, T. 2003, ApJ, 598, 886 (U03)

Ueda, Y., Hiroi, K., Isobe, N., et al. 2011, PASJ, 63S, 937

Ueda, Y., Ishisaki, Y., Takahashi, T., Makishima, K., \& Ohashi, T. 2001, ApJS, 133,1

Ueda, Y., Ishisaki, Y., Takahashi, T., Makishima, K., \& Ohashi, T. 2005, ApJS, 161,185

Ueda, Y., Takahashi, T., Inoue, H., et al. 1998, Natur, 391, 866

Ueda, Y., Takahashi, T., Inoue, H., et al. 1999, ApJ, 518, 656

Ueda, Y., Watson, M. G., Stewart, I. M., et al. 2008, ApJS, 179, 124

Vasudevan, R. V., Brandt, W. N., Mushotzky, R. F., et al. 2013, ApJ, 763, 111

Vika, M., Driver, S. P., Graham, A. W., \& Liske, J. 2009, MNRAS, 400, 1451

Vito, F., Vignali, C., Gilli, R., et al. 2013, MNRAS, 428, 354

Wilman, R. J., \& Fabian, A. C. 1999, MNRAS, 309, 862

Winter, L. M., Mushotzky, R. F., Reynolds, C. S., \& Tueller, J. 2009a, ApJ, 690, 1322

Winter, L. M., Mushotzky, R. F., Terashima, Y., \& Ueda, Y. 2009b, ApJ, 701,644

Xue, Y. Q., Luo, B., Brandt, W. N., et al. 2011, ApJS, 195, 10

Yang, Y., Mushotzky, R. F., Steffen, A. T., Barger, A. J., \& Cowie, L. L. 2004, AJ, 128, 1501

Yencho, B., Barger, A. J., Trouille, L., \& Winter, L. M. 2009, ApJ, 698, 380

Yu, Q., \& Tremeine, S. 2002, MNRAS, 335, 965

Zdziarski, A. A., Johnson, W. N., Done, C., et al. 1995, ApJL, 438, L63

Zdziarski, A. A., Poutanen, J., \& Johnson, W. N. 2000, ApJ, 542, 703 